\title{
Basicity-Tuned Reactivity: diaza-[1,2]-Wittig versus diaza-[1,3]-Wittig Rearrangements of 3,4-Dihydro-2H-1,2,3-benzothiadiazine 1,1-Dioxides
}

Imre Gyüjtő,a,b Márta Porcs-Makkay, ${ }^{a}$ Gergő Szabó, ${ }^{b}$ Zsolt Kelemen, ${ }^{b}$ Gyöngyvér Pusztai, ${ }^{a}$ Gábor Tóth, ${ }^{a}$ András Dancsó, ${ }^{a}$ Judit Halász, ${ }^{a}$ Gyula Simig, ${ }^{a}$ Balázs Volk, ${ }^{a,{ }^{*}}$ László Nyulászib,*

\section{Table of content}

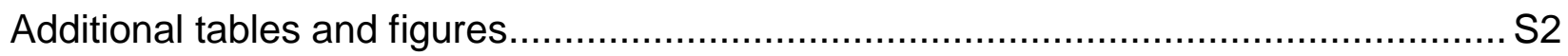

Detailed NMR structure elucidation of compounds $\mathbf{2} \mathbf{a}, \mathbf{3} \mathbf{a}$, trans-3b and cis-3b, compounds present in $t$-BuOK/[D6]DMSO reaction mixtures, and structure elucidation of [D]3a S13

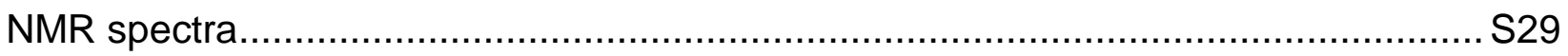

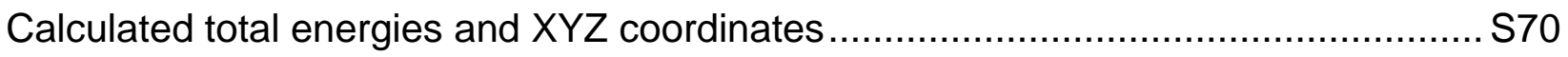

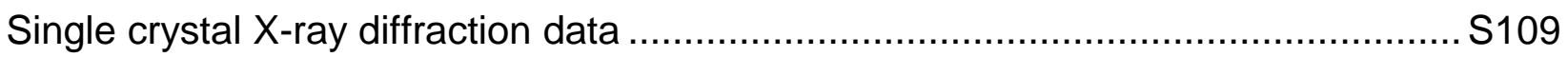




\section{Additional tables and figures}

Table S1. Experimental study of various bases used for the deprotonation of 1a<smiles>CC(=O)N1C(C)c2ccc(Cl)c(Cl)c2S(=O)(=O)N1C</smiles>

1a<smiles>CC(=O)NC1(C)c2ccc(Cl)c(Cl)c2S(=O)(=O)N1C</smiles>

\begin{tabular}{|c|c|c|}
\hline Base & Product & Yield (\%) \\
\hline$t-\mathrm{BuOK}$ & $\mathbf{2 a}$ & 90 \\
\hline $\mathrm{NaOH}$ & $\mathbf{2 a}$ & 90 \\
\hline $\mathrm{NaOMe}$ & $\mathbf{2 a}$ & 76 \\
\hline $\mathrm{DBU}$ & $\mathbf{2 a}$ & 95 \\
\hline \hline $\mathrm{DIPEA}$ & $\mathbf{1 a}$ & 95 \\
\hline $\mathrm{NaOAc}$ & $\mathbf{1 a}$ & 80 \\
\hline $\mathrm{EMIM}^{\star}-\mathrm{OAc}$ & $\mathbf{1 a}$ & $91^{\text {** }}$ \\
\hline
\end{tabular}

Reagents and reaction conditions: 1 a $(0.6 \mathrm{mmol})$, base (2 equiv), THF $(3 \mathrm{~mL}), 25^{\circ} \mathrm{C}, 30 \mathrm{~min}$, quenching with water, evaporation of THF, crystallization.

* EMIM stands for 1-ethyl-3-methylimidazolium cation.

${ }^{* *}$ With ${ }^{1} \mathrm{H}$ NMR, traces of $2 \mathrm{a}$ could be detected in the crude product.

Table S2. Comparison of the M06-2X/6-31+G* gas-phase acidity of 1 a at position $\mathrm{C}(4)$ with that of other weak acids

\begin{tabular}{|c|c|}
\hline $\mathbf{H B}$ & $\boldsymbol{\Delta} \mathbf{G}_{\text {gas }}[\mathbf{k c a l} / \mathbf{m o l}]$ \\
\hline $\mathrm{H}_{2} \mathrm{O}$ & 382.7 \\
\hline $\mathrm{MeOH}$ & 370.3 \\
\hline$t-\mathrm{BuOH}$ & 365.1 \\
\hline 1a at $\mathrm{C}(4)$ atom & 329.9 \\
\hline $\mathrm{AcOH}$ & 329.2 \\
\hline
\end{tabular}

Gas-phase acidity values in Scheme 3 and Table S2 were obtained as follows: neutral and deprotonated forms of $1 \mathrm{a}$ (at all possible positions), $\mathrm{H}_{2} \mathrm{O}, \mathrm{MeOH}, t-\mathrm{BuOH}$ and $\mathrm{AcOH}$ were optimized at M06-2X/6-31+G* level of theory. Then free energies were calculated at the same level with fine grid and $\mathrm{G}\left(\mathrm{H}^{+}(\right.$gas $\left.)\right)=-6.28 \mathrm{kcal} / \mathrm{mol}$. Deprotonation Gibbs free energies were calculated according to the following equation:

$$
\Delta G_{\text {gas }}=G\left(B^{-}(\text {gas })\right)+G\left(H^{+}(\text {gas })\right)-G(H B(\text { gas }))
$$


Noteworthy features in the ${ }^{1} \mathrm{H}$ NMR spectrum of $\mathbf{I V}^{2-}$ depicted in Figure 1 in the manuscript: (i) the partially hindered rotation of the amide moiety results in a characteristic line broadening; (ii) the intensity of the $-\mathrm{C}(=\mathrm{O})-\mathrm{CH}_{3}$ signal is only ca. $1.25 \mathrm{H}$ (instead of $3 \mathrm{H}$ ), revealing a $\mathrm{H}-\mathrm{D}$ exchange with $\left[\mathrm{D}_{6}\right] \mathrm{DMSO}$ under these strongly basic conditions, also supported by LC-MS (ESI) measurements (Figure $S 1)$. When a sample of 1a / 6 equiv $t$-BuOK / DMSO reaction mixture was analyzed with ${ }^{1} \mathrm{H}$ NMR in $\left[\mathrm{D}_{6}\right] \mathrm{DMSO}$, the $-\mathrm{C}(=\mathrm{O})-$ $\mathrm{C}_{3} 3$ signal intensity remained about $3 \mathrm{H}$ in the ${ }^{1} \mathrm{H}$ NMR spectrum (Figure $\mathrm{S} 2$ ).

Procedure: To a mixture of 1a $(0.6 \mathrm{mmol}, 194 \mathrm{mg})$ dissolved in DMSO $(3 \mathrm{~mL}), t$-BuOK (3.6 mmol, $404 \mathrm{mg}$ ) was added at $25^{\circ} \mathrm{C}$ and stirred for $10 \mathrm{~min}$. A sample was analyzed by ${ }^{1} \mathrm{H}$ NMR (Figure S2), which contained IV ${ }^{2-}$ dominantly. The residue was quenched with water $(10 \mathrm{~mL})$, extracted with DCM $(1 \times 50,3 \times 25 \mathrm{~mL})$, the combined organic layer was dried over $\mathrm{MgSO}_{4}$ and evaporated to give $159 \mathrm{mg}(85 \%)$ product identified as a mixture of $\mathbf{2 a}, \mathbf{3} \mathbf{a}$ and $\mathbf{5 a}$ in a ratio of ca. 0.17:1.05:1.00 (Figure S3).
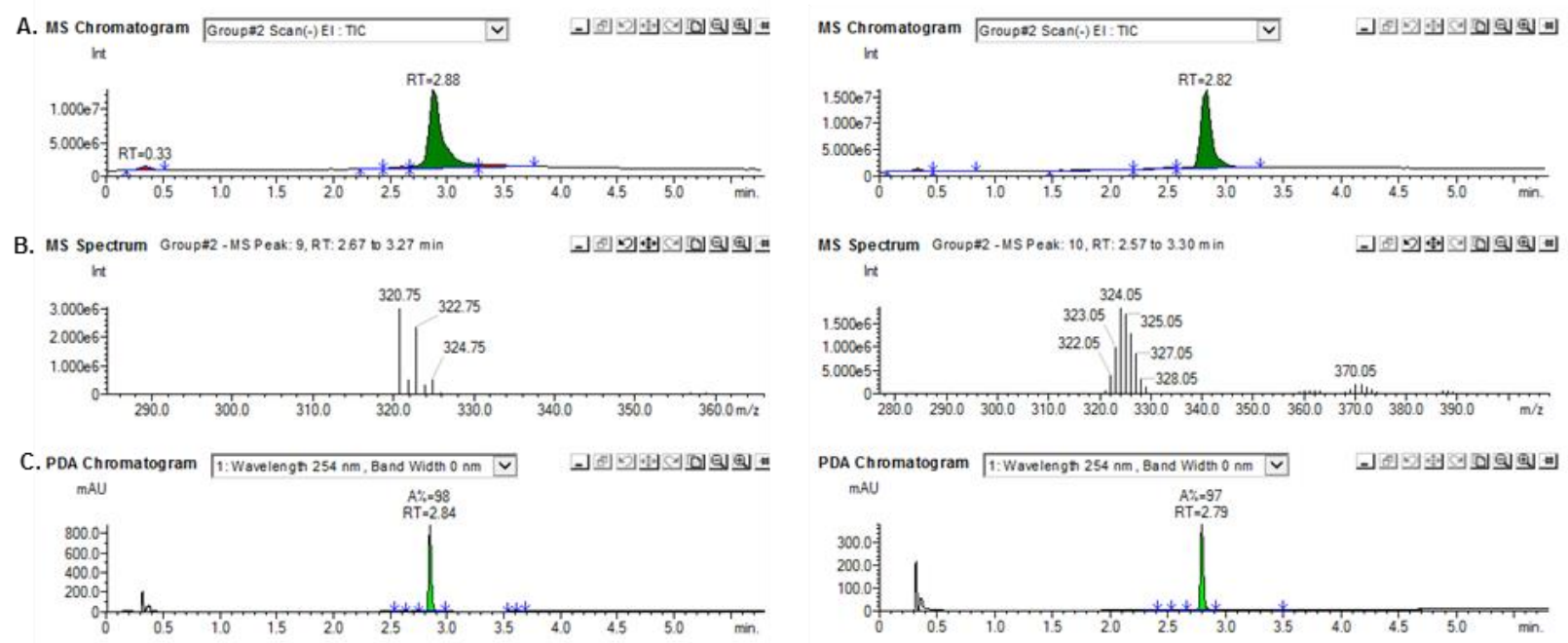

Figure S1. LC-MS analysis of the reaction of 1a with 6 equiv $t$-BuOK in DMSO (left) or in [D6]DMSO (right) 


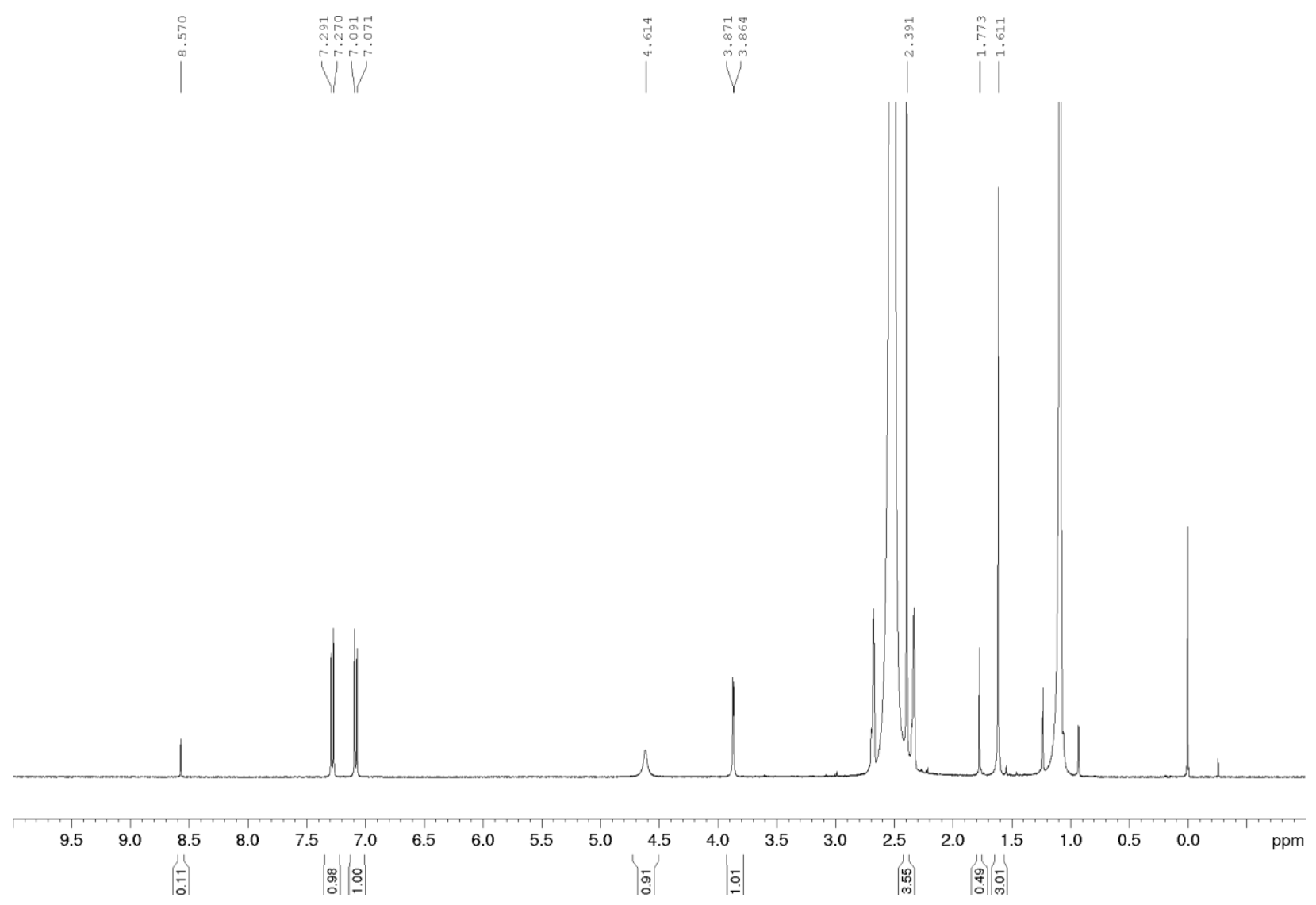

Figure S2. ${ }^{1} \mathrm{H}$ NMR analysis of a sample from 1a / 6 equiv $t$-BuOK / DMSO reaction mixture, measured in $\left[D_{6}\right]$ DMSO 


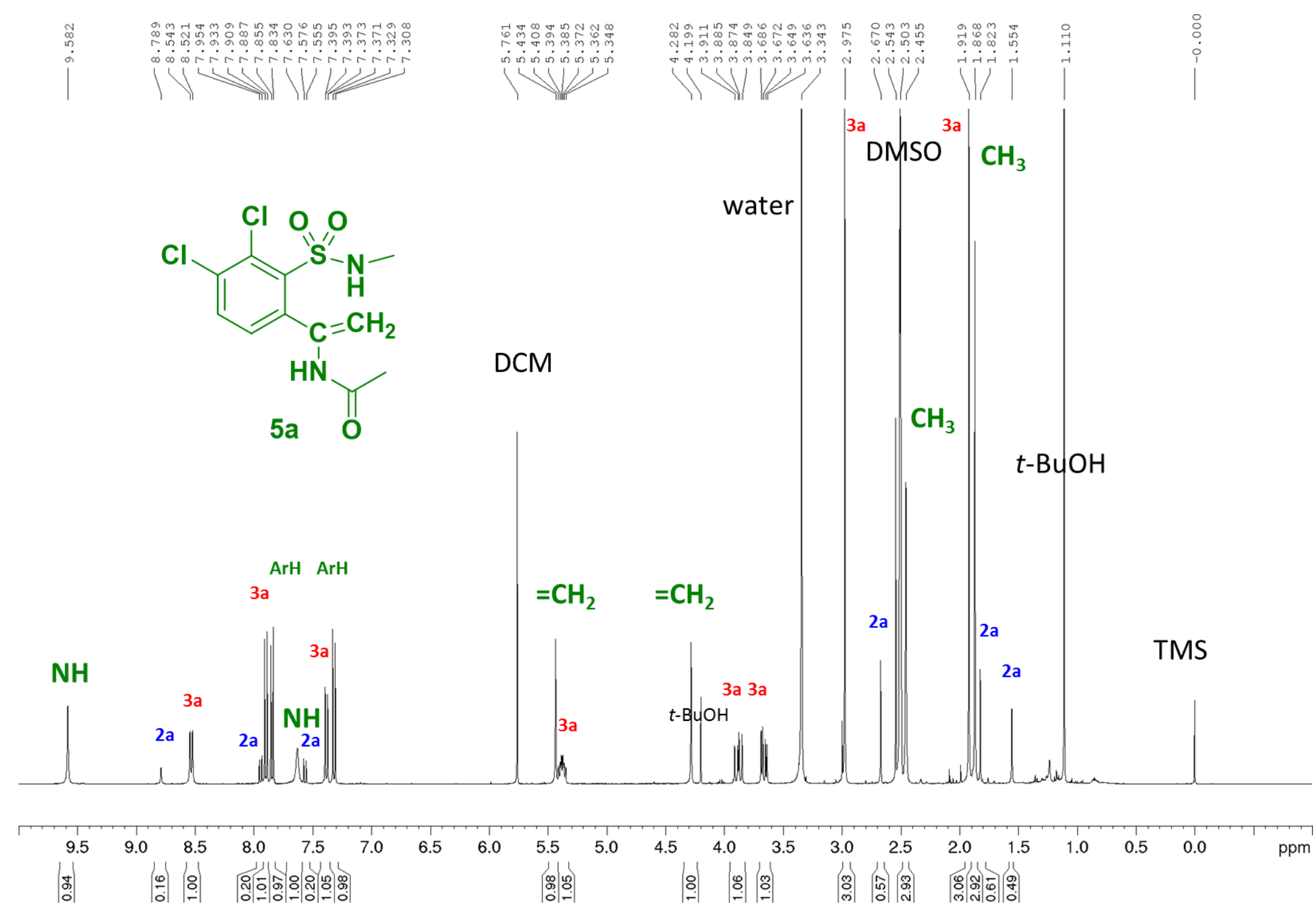

Figure S3. ${ }^{1} \mathrm{H}$ NMR spectrum of an extracted mixture containing enamide 5a 
<smiles>CC(=O)N1[CH]c2ccc(Cl)c(Cl)c2S(=O)(=O)N1C</smiles>

$I^{-}$

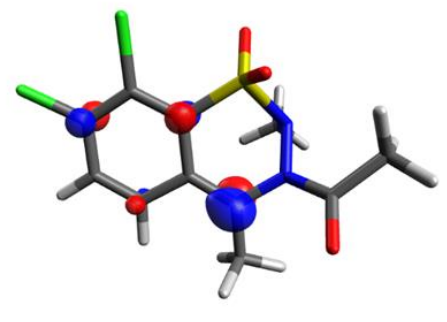

I- HOMO<smiles>CNS(=O)(=O)c1c(C(C)=NC(C)=O)ccc(Cl)c1Cl</smiles>

III $^{-}$

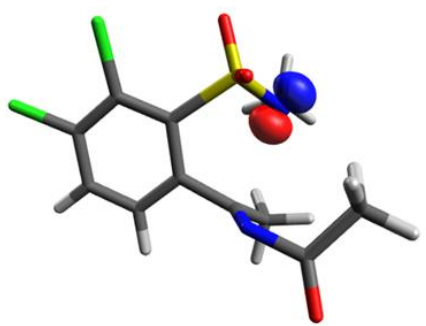

$\mathrm{III}^{-}$HOMO

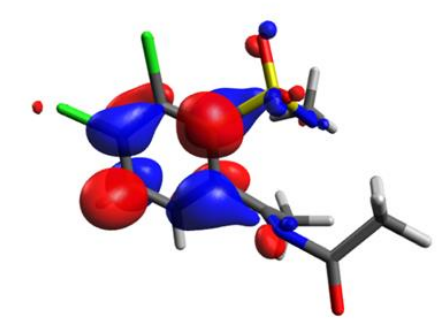

III- LUMO+1<smiles>C=C(NC(C)=O)c1ccc(Cl)c(Cl)c1S(=O)(=O)NC</smiles>

$\mathrm{V}^{-}$

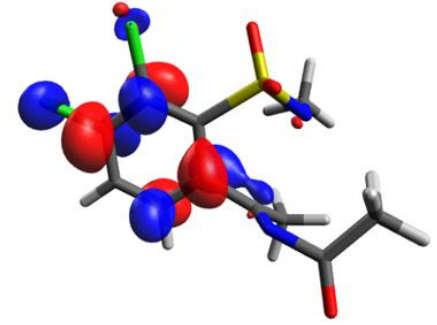

III- LUMO+2

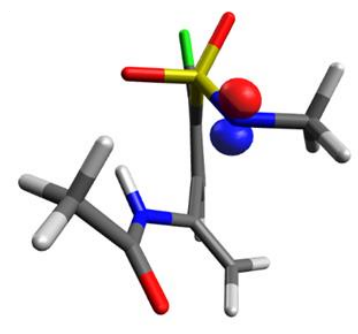

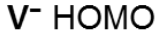

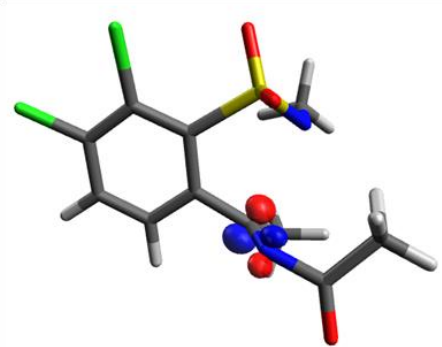

III- LUMO+5

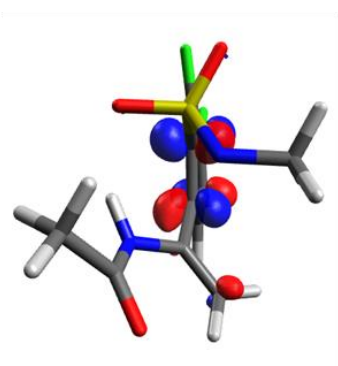

V' LUMO $^{-}$

Figure S4. Selected Kohn-Sahm molecular orbitals of $\mathbf{I}^{-}$, III and $\mathbf{V}^{-}$at $M 06-2 X / 6-31+G^{*}$ level of theory 


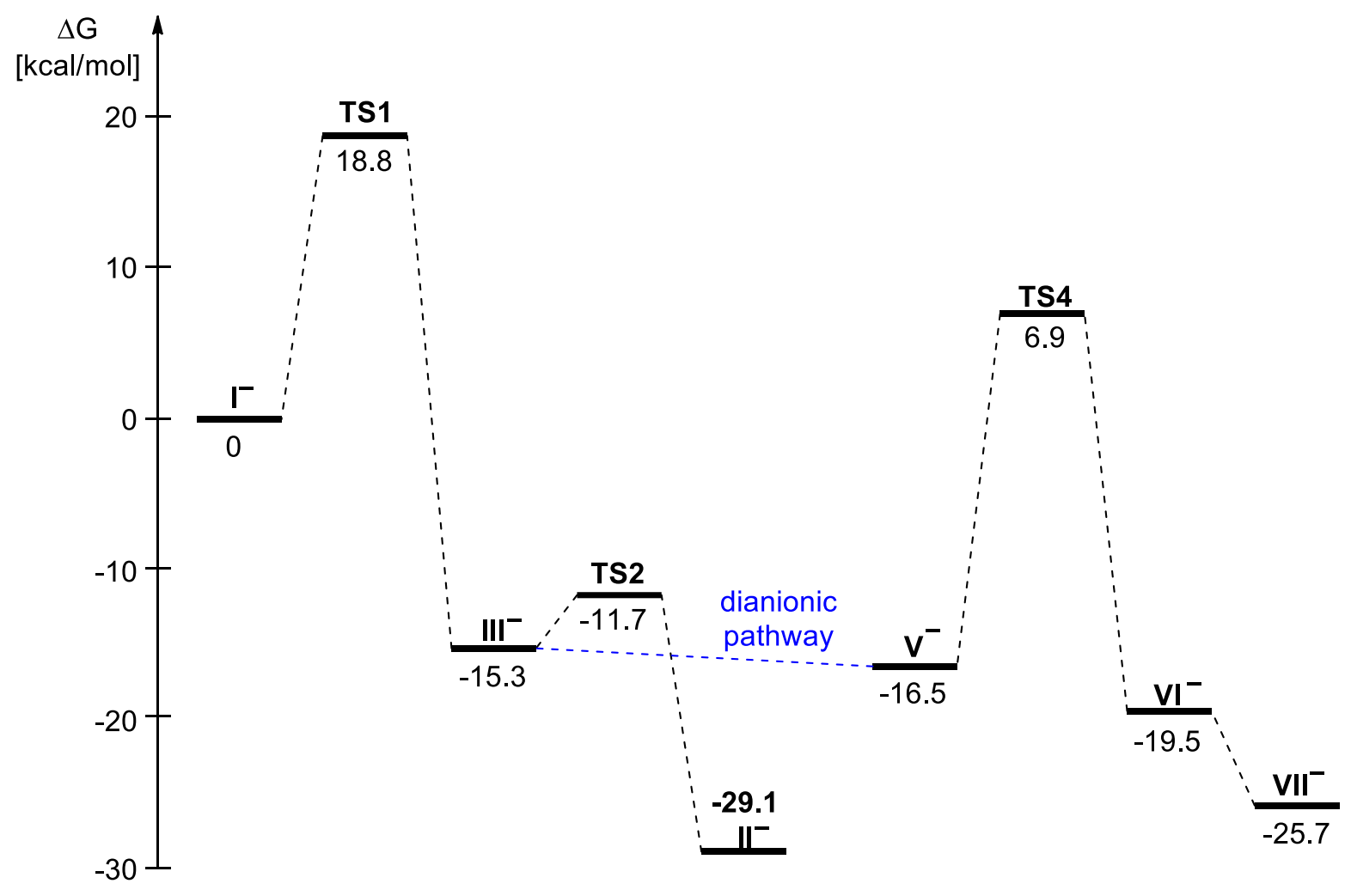

Figure S5. Gibbs free energy profile diagram of the reaction mechanism at M06-2X/6$31+\mathrm{G}^{*}$ (smd: THF) level of theory depicted in Scheme 4 


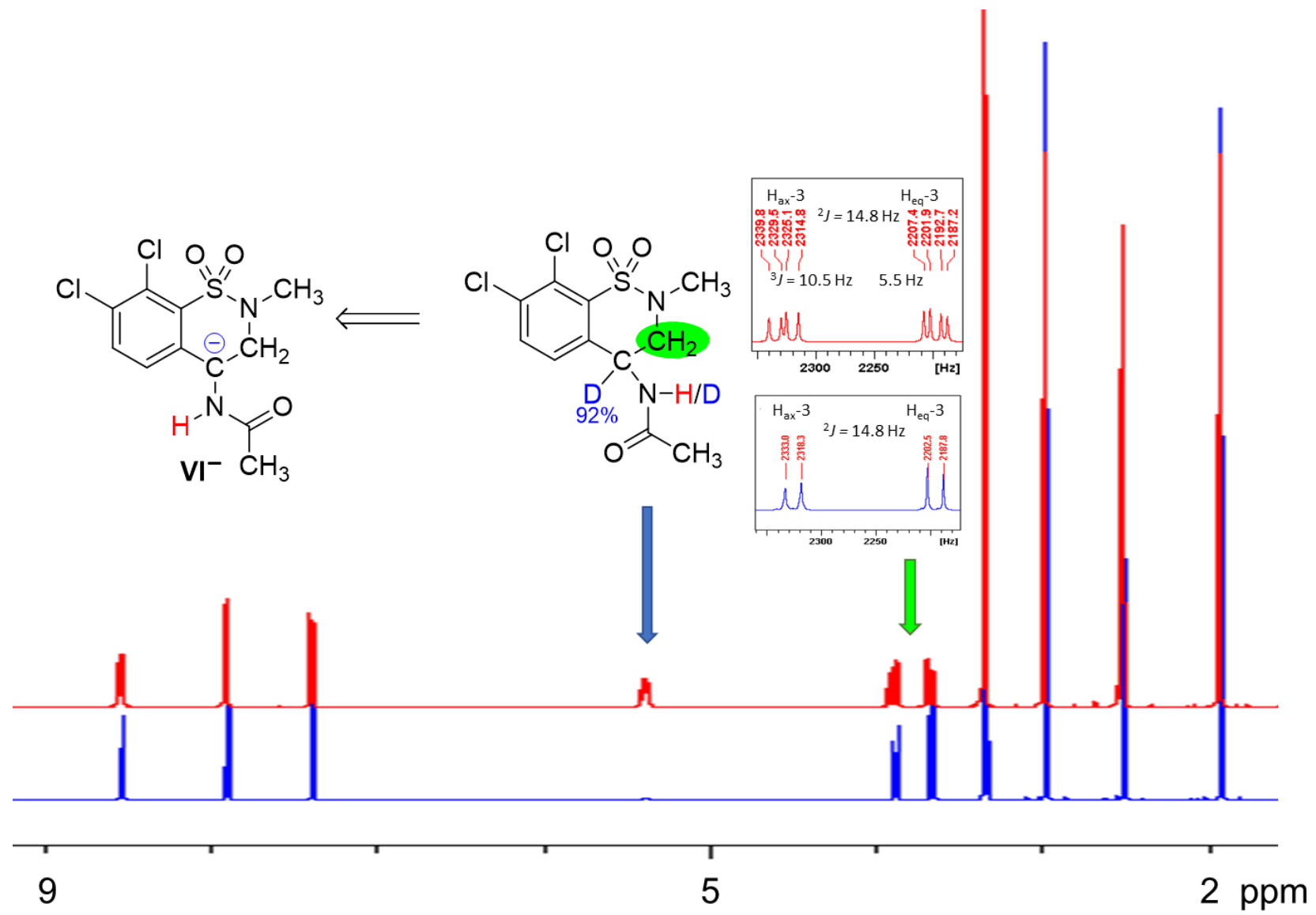

Figure S6. ${ }^{1} \mathrm{H}$ NMR spectra in $\left[\mathrm{D}_{6}\right] \mathrm{DMSO}$ of $\mathbf{3 a}$ (red) and [D]3a (blue) after trapping carbanion $\mathrm{VI}^{-}$with $\mathrm{D}_{2} \mathrm{O}$ 
To the solution of $10(0.6 \mathrm{mmol}, 153 \mathrm{mg})$ in THF ( $3 \mathrm{~mL})$ was added $t$-BuOK $(1.2 \mathrm{mmol}, 2$ equiv, $135 \mathrm{mg}$ ) at $25^{\circ} \mathrm{C}$ and stirred for $30 \mathrm{~min}$. Then it was quenched with water $(10 \mathrm{~mL})$, THF was removed in vacuo, and extracted with DCM $(2 \times 30 \mathrm{~mL})$. The combined organic layer was dried over $\mathrm{MgSO}_{4}$ and evaporated to give $133 \mathrm{mg}(87 \%)$ product, identified as a mixture of 20 and the corresponding enamide 50 in a molar ratio of ca. 0.13:1.00 (Figures S7 and S8).

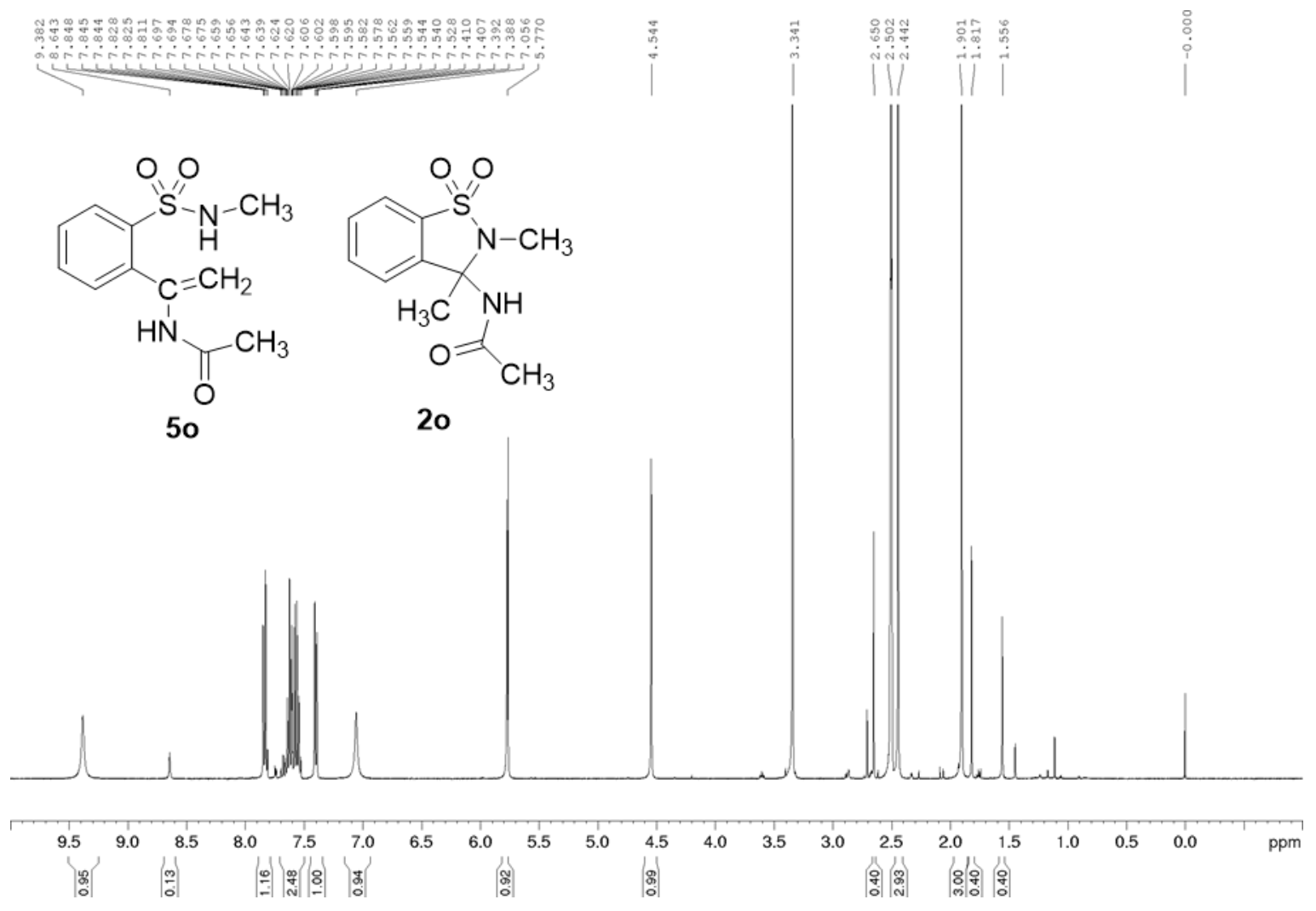

Figure S7. ${ }^{1} \mathrm{H}$ NMR spectrum of $\mathbf{2 0}$ (minor product) besides the corresponding enamide 50 (major product) 


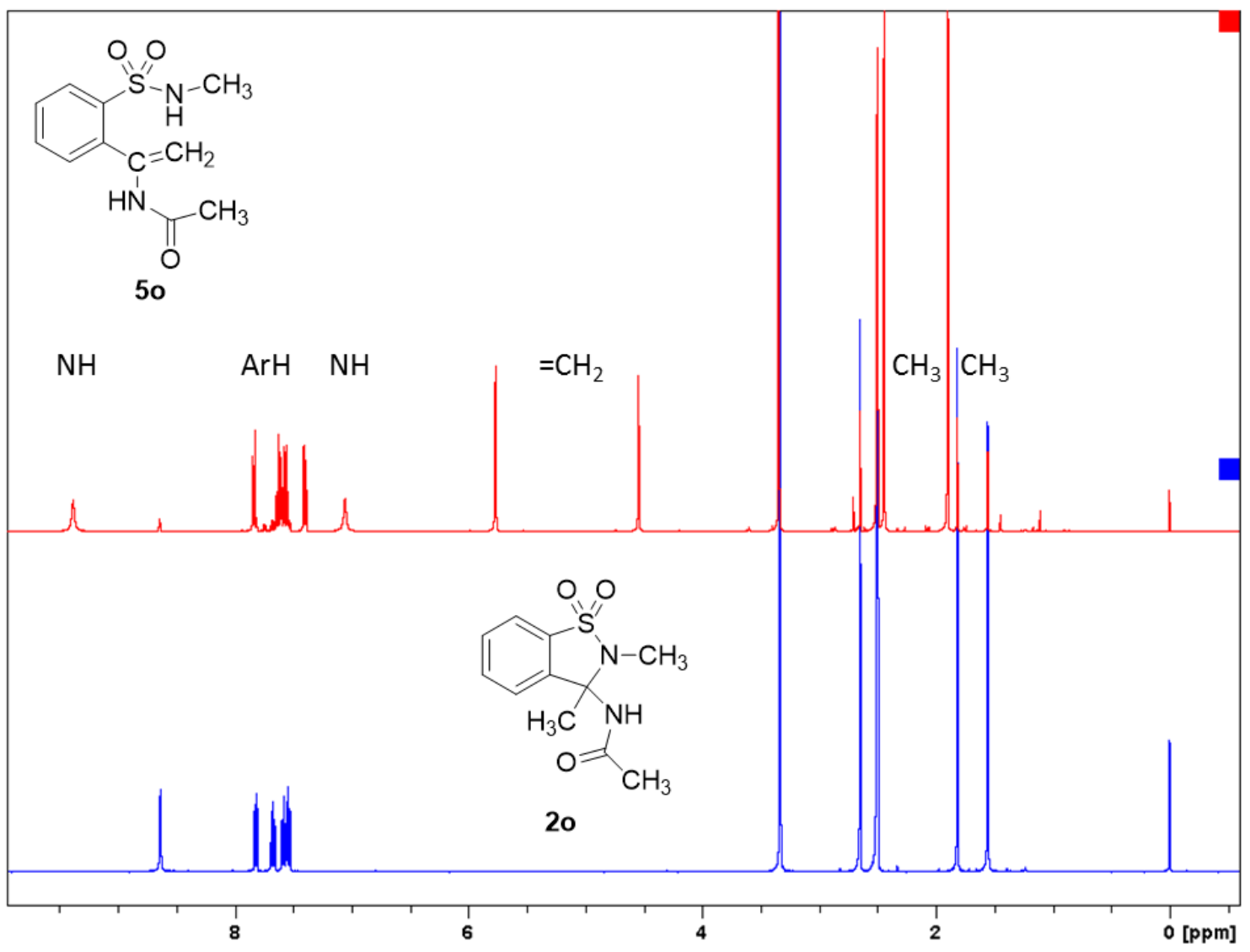

Figure S8. ${ }^{1} \mathrm{H}$ NMR spectrum of $\mathbf{2 0}$ in a mixture with enamide $\mathbf{5 0}$ (above) and in its pure form (below) 


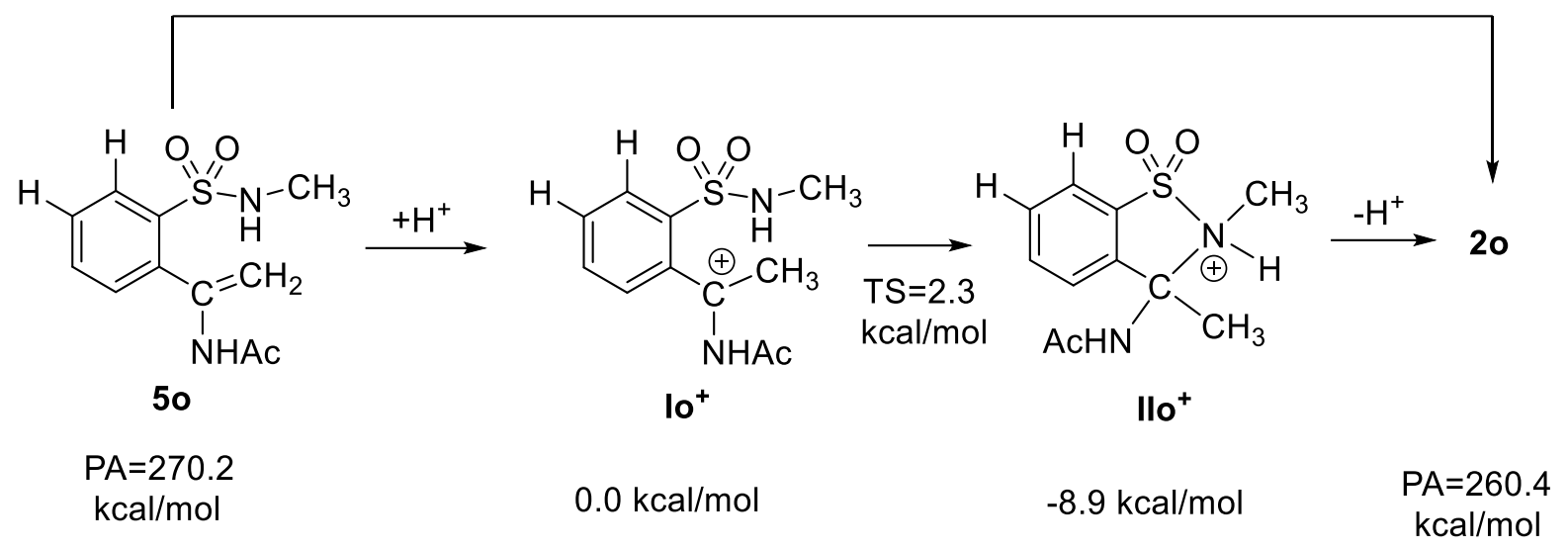

Figure S9. Calculated reaction mechanism of the proton-catalyzed transformation of enamide 50 to 20 (at M06-2X/6-31+G* SMD:THF level of theory)

$\Delta \mathrm{E}=-19.5 \mathrm{kcal} / \mathrm{mol}$

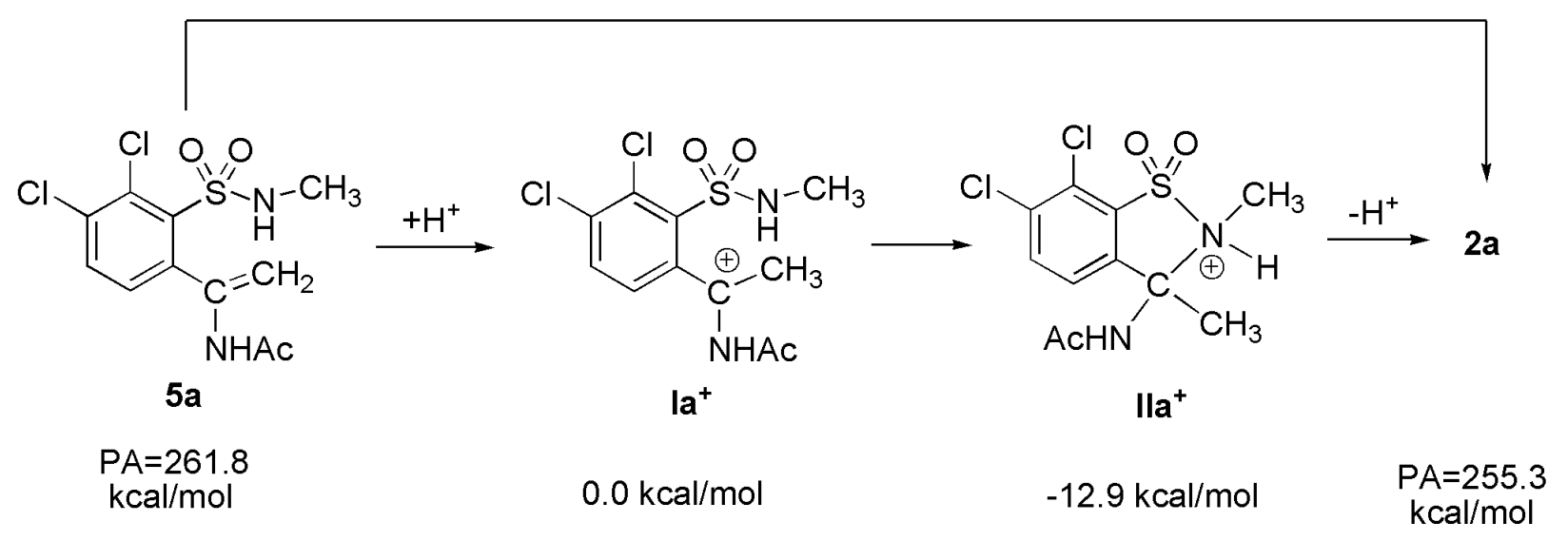

Figure S10. Calculated reaction mechanism of the proton-catalyzed transformation of enamide $\mathbf{5 a}$ to $\mathbf{2 a}$ (at M06-2X/6-31+G* SMD:THF level of theory)

Unfortunately, all attempts to localize the transition state between $\mathbf{l a}^{+}$and $\mathrm{lla}^{+}$failed in our hand. In accordance with this, scan calculations (by decreasing the $\mathrm{N}-\mathrm{C}$ distance stepwise by $0.1 \AA$ ) showed that the energy of the system decreased continuously, indicating no significant barrier during this process. In case of $\mathbf{5 0}$, however, we could localize the corresponding TS and the process exhibited a tiny barrier of $2.3 \mathrm{kcal} / \mathrm{mol}$. Altogether, it is easily understandable that upon protonation the reaction proceeds with a wide range of substituents easily to the thermodynamically stable product 2.

Experimental evidence to support the mechanism above: acidification of the enamide containing reaction mixture $\left(\mathbf{I V}^{2-} / \mathbf{V}^{-} / \mathbf{5 a}\right)$ gave $\mathbf{2 a}$ : 
To the solution of $1 \mathrm{a}(0.6 \mathrm{mmol}, 194 \mathrm{mg})$ in THF $(3 \mathrm{~mL})$ was added $t$-BuOK $(3.6 \mathrm{mmol}$, $404 \mathrm{mg}$ ) at $25^{\circ} \mathrm{C}$ and stirred for $30 \mathrm{~min}$. After that, THF was evaporated, and the residue was quenched with aq $\mathrm{HCl}(5 \mathrm{w} / \mathrm{w} \%, 5 \mathrm{~mL})$. Then it was cooled to $5{ }^{\circ} \mathrm{C}$, the precipitated product was filtered and washed with water to give 2a. Yield: $178 \mathrm{mg}(92 \%)$.

Table S3. Testing of various methods and levels of theory

\begin{tabular}{|c|c|c|c|c|c|}
\hline E [kcal $/ \mathrm{mol}]$ & optimization (smd: THF) & \multicolumn{4}{|c|}{ single point calculation (smd: THF) } \\
\hline $\begin{array}{l}\text { method } \\
\text { level of theory }\end{array}$ & $\begin{array}{l}M 06-2 X \\
6-31+G^{*}\end{array}$ & $\begin{array}{c}\text { B3LYP } \\
6-31+G^{*}\end{array}$ & $\begin{array}{c}\omega B 97 X-D \\
6-31+G^{*}\end{array}$ & $\begin{array}{c}\text { M06-2X } \\
\text { aug-cc-pVTZ }\end{array}$ & $\begin{array}{c}M 06-2 X \\
6-311++G^{* *}\end{array}$ \\
\hline $\mathrm{I}^{-}$ & 0.0 & 0.0 & 0.0 & 0.0 & 0.0 \\
\hline TS1 & 19.6 & 13.2 & 19.1 & 20.0 & 20.0 \\
\hline $\mathrm{III}^{-}$ & -13.2 & -18.1 & -16.0 & -14.9 & -13.6 \\
\hline TS2 & -9.9 & -15.8 & -12.3 & -10.9 & -10.0 \\
\hline $\mathrm{II}^{-}$ & -29.5 & -30.9 & -32.6 & -29.7 & -28.7 \\
\hline $\mathbf{V}^{-}$ & -16.4 & -18.1 & -16.4 & -20.1 & -18.6 \\
\hline TS3 & 8.2 & 2.4 & 7.6 & 6.7 & 6.8 \\
\hline $\mathrm{VI}^{-}$ & -20.0 & -20.8 & -20.5 & -22.2 & -21.5 \\
\hline $\mathrm{VII}^{-}$ & -27.6 & -28.7 & -29.6 & -27.7 & -26.9 \\
\hline $\mathrm{IV}^{2-}$ & 0.0 & 0.0 & 0.0 & 0.0 & 0.0 \\
\hline TS4 & 29.8 & 27.7 & 31.0 & 31.6 & 30.2 \\
\hline VIIII- & -2.1 & 1.8 & -0.8 & -0.7 & -1.9 \\
\hline
\end{tabular}

Optimization was performed at M06-2X/6-31+G* (THF: smd). Single point calculation at the same level employing B3LYP showed moderate differences at TS1, whereas $\omega B$ 97X$D$ gave similar results. Using larger basis sets (aug-cc-pVTZ or $6-311++G^{* *}$ ) afforded only slight changes in the relative energy values, thus we used the $6-31+G^{*}$ basis.

Direct tautomerization of $\mathrm{III}^{-}$to $\mathbf{V}^{-}$was initially investigated at $B 3 \mathrm{LYP} / 6-31+\mathrm{G}^{*}$, and gave a barrier of $\Delta \mathrm{G}^{\#}=79.2 \mathrm{kcal} / \mathrm{mol}$. 
Detailed NMR structure elucidation of compounds $2 a, 3 a$, trans-3b and cis-3b, compounds present in $t-\mathrm{BuOK}_{/}\left[\mathrm{D}_{6}\right] \mathrm{DMSO}$ reaction mixtures, and structure elucidation of $[D] 3 a$

Compound 2a, ${ }^{1} \mathrm{H}$ and DEPTQ

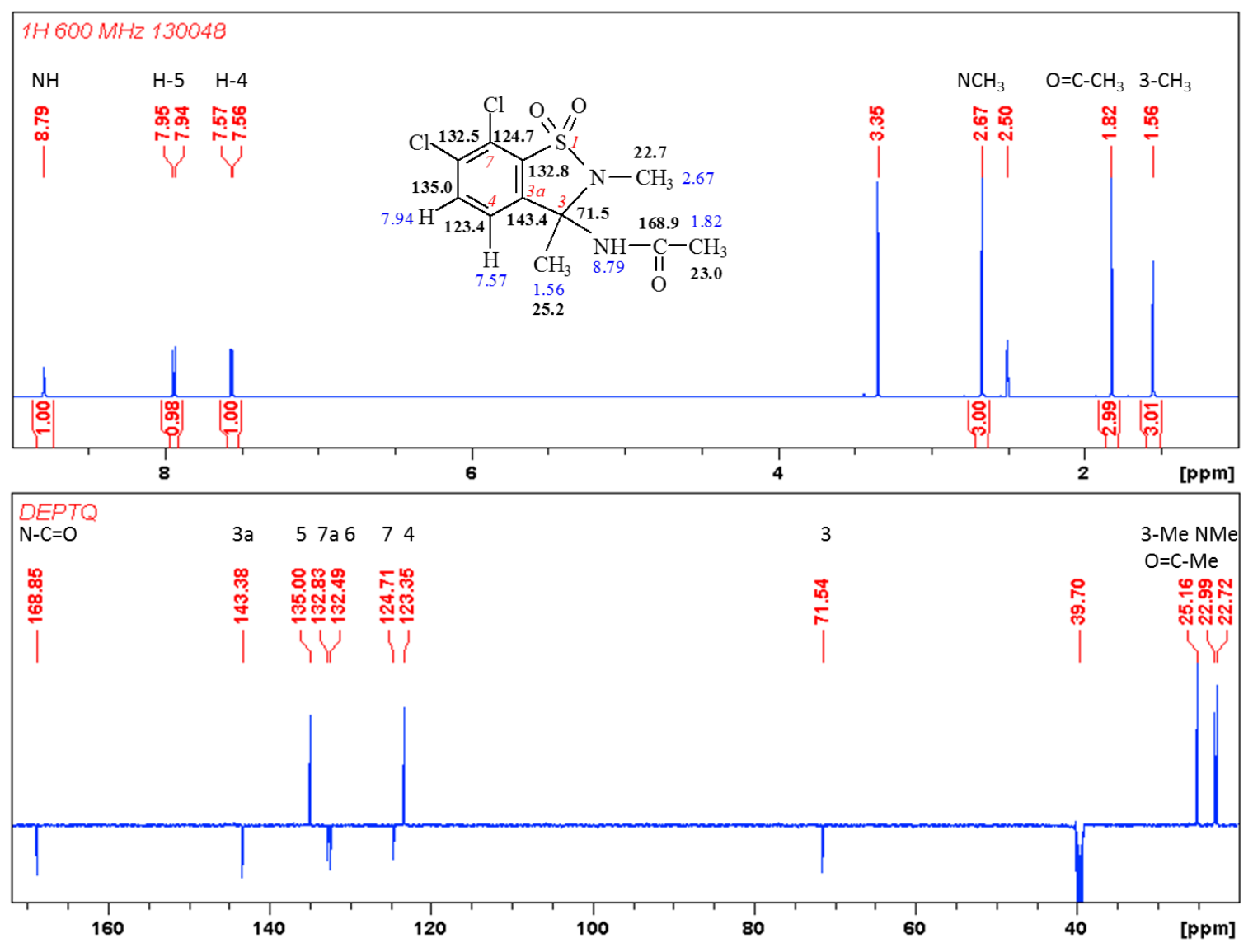


Compound 2a, HSQC and HMBC

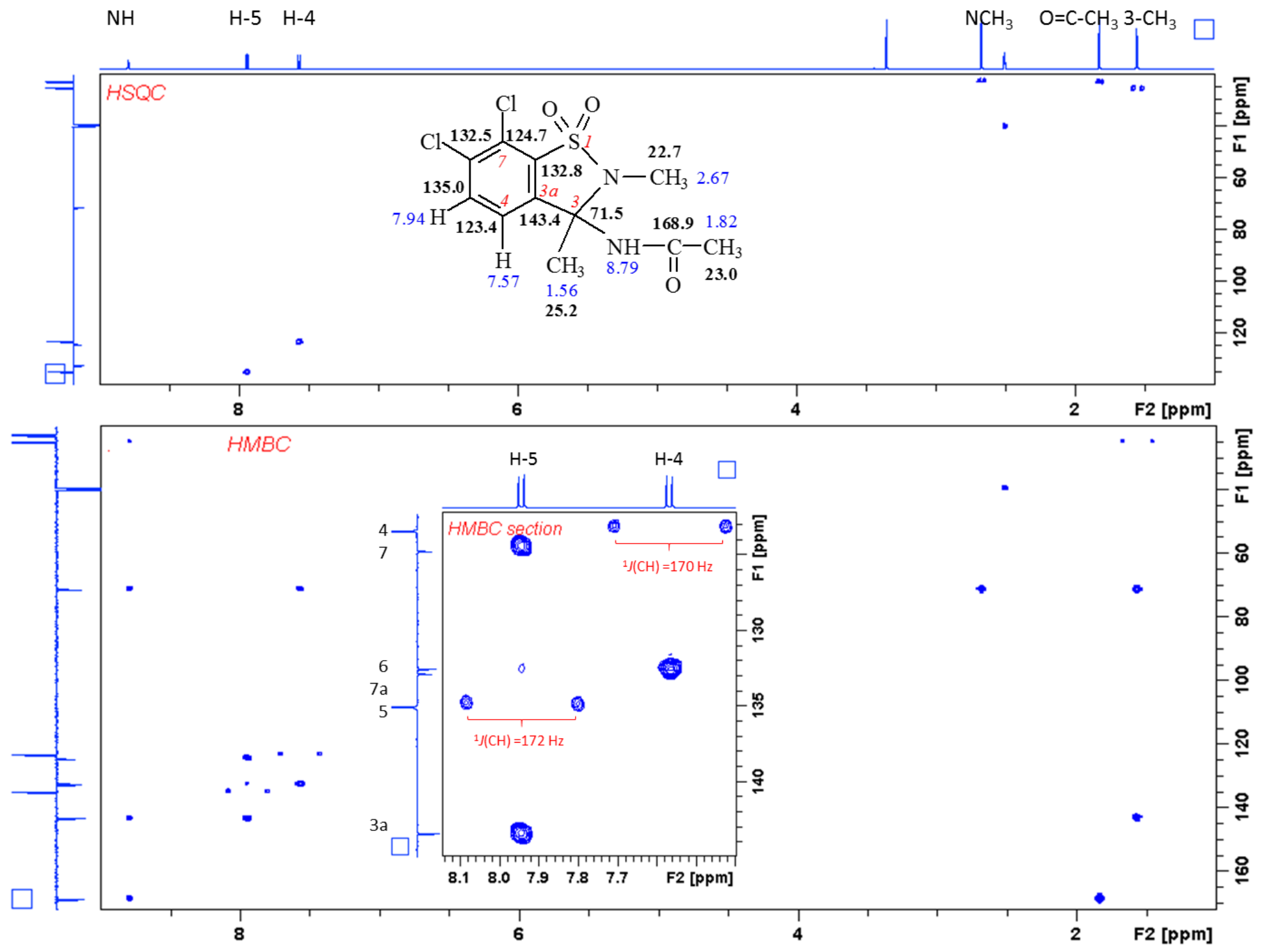


Compound $3 \mathrm{a},{ }^{1} \mathrm{H}$ and HSQC

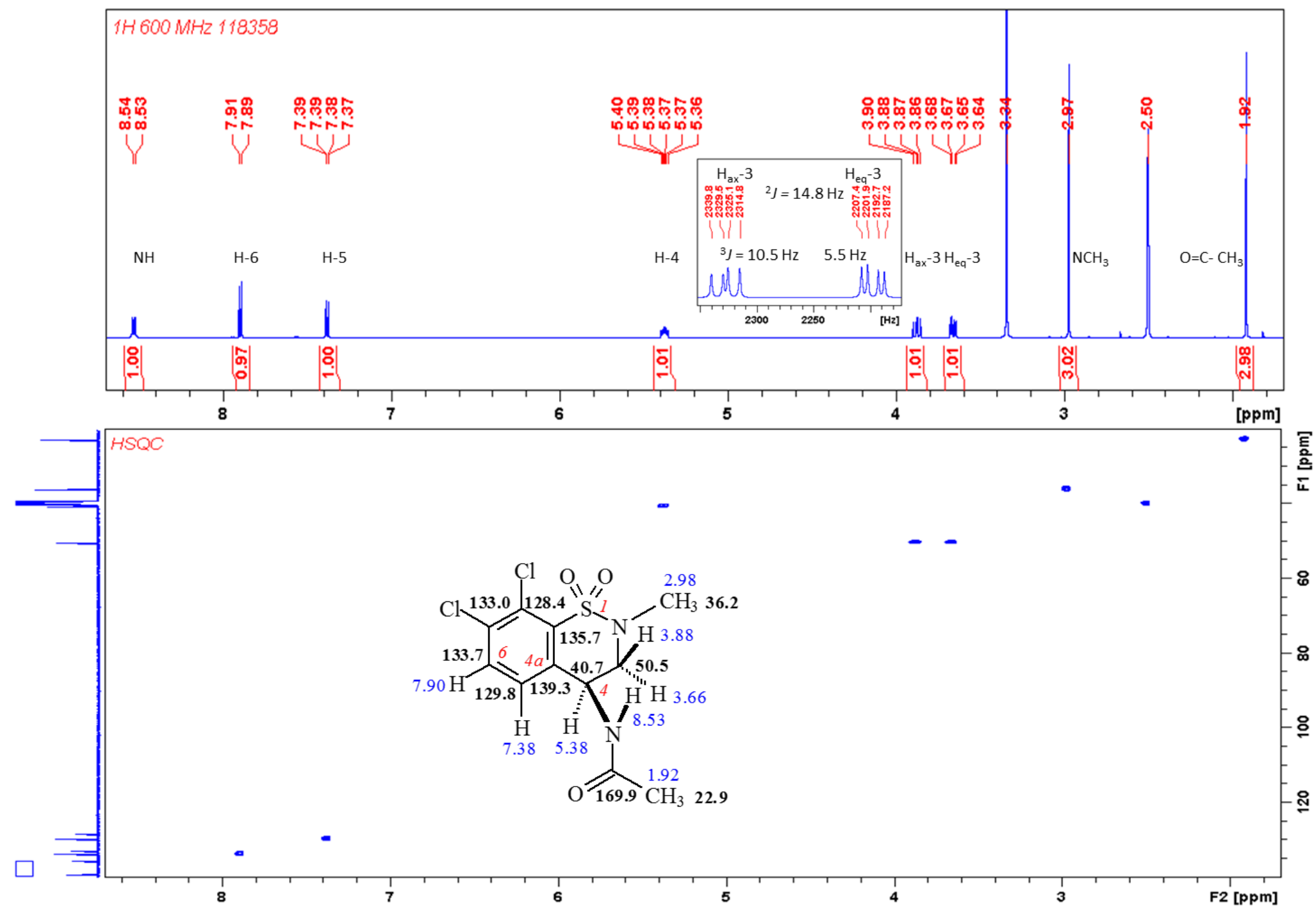


Compound $3 \mathbf{3 a},{ }^{13} \mathrm{C}$ and HMBC

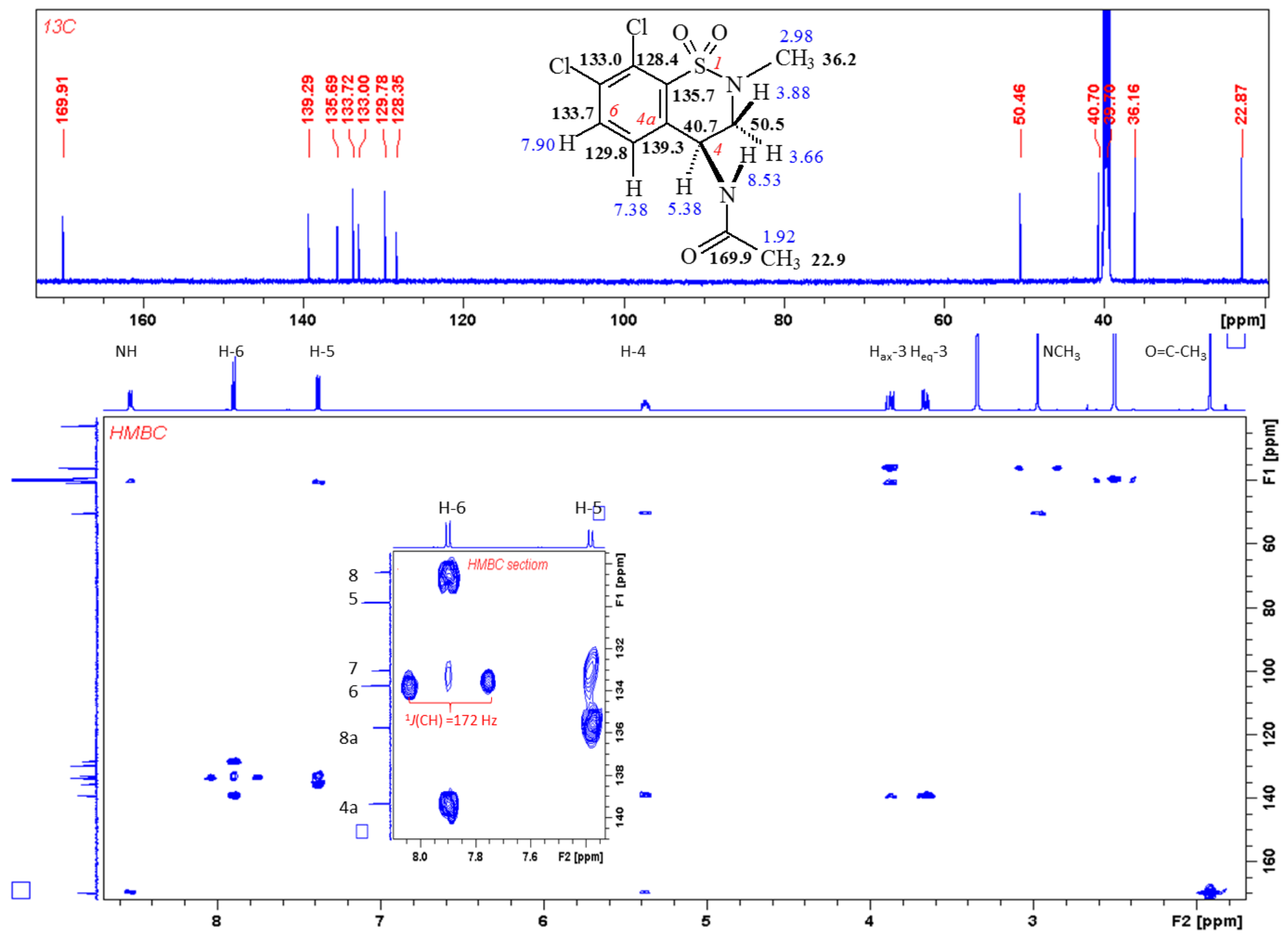


Compound trans-3b, ${ }^{1} \mathrm{H}$ and edHSQC

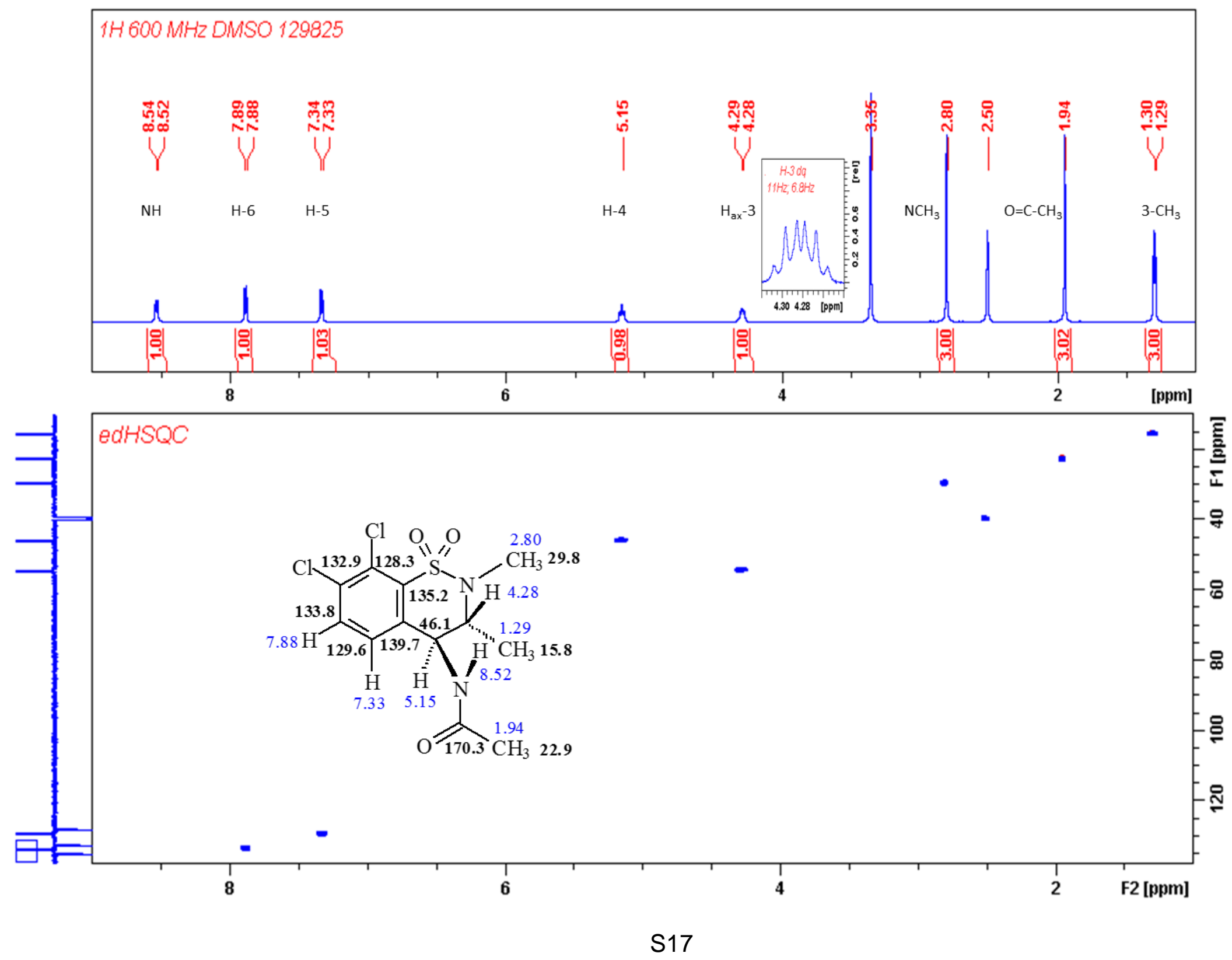


Compound trans-3b, DEPTQ and HMBC
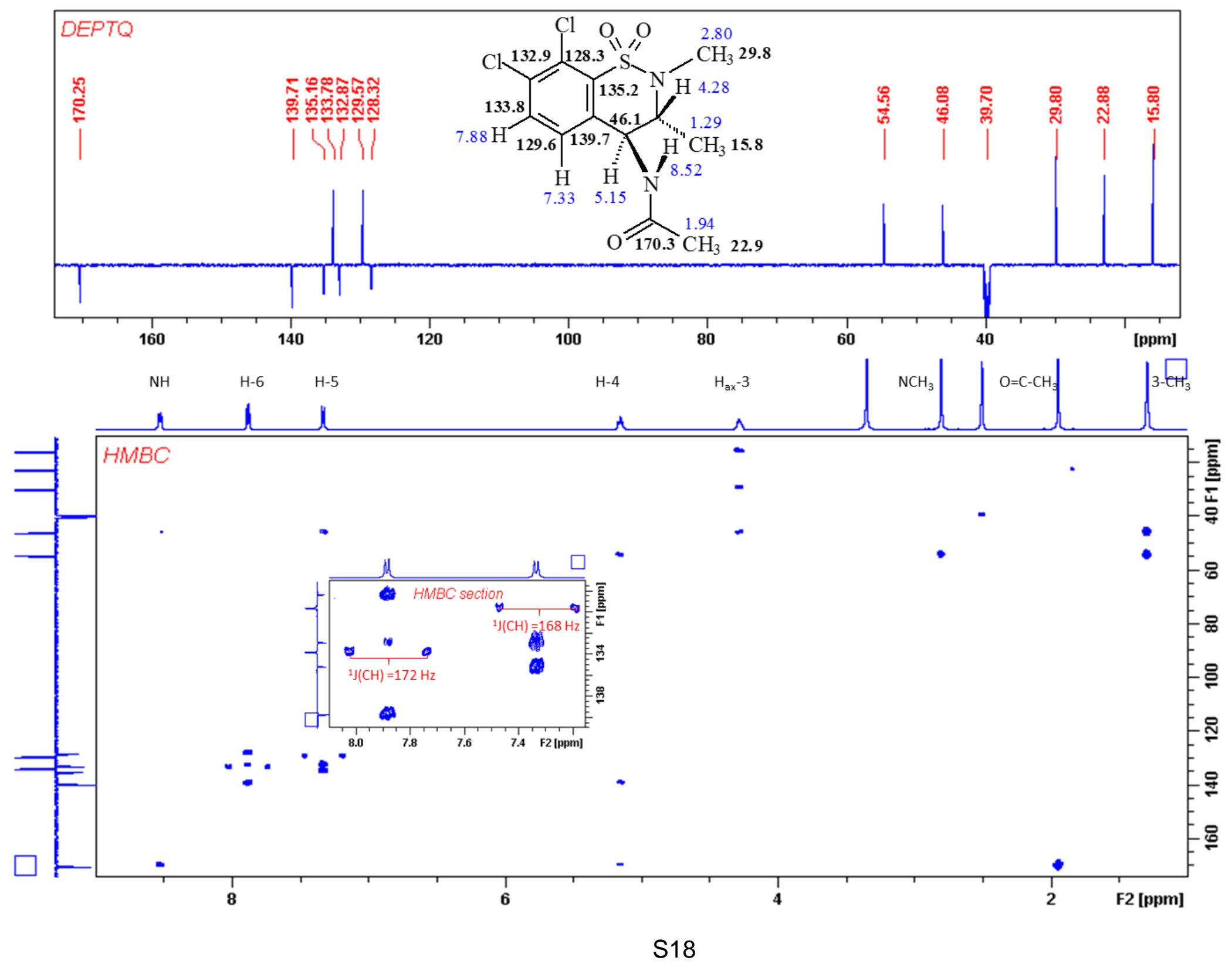
Compound trans-3b and stereostructure supported by ${ }^{1} \mathrm{H}$, selNOE on $\mathrm{H}-4, \mathrm{NCH}_{3}, 3-\mathrm{CH}_{3}$, selROE on $\mathrm{H}-3$ and DFT geometry optimalization (from bottom)
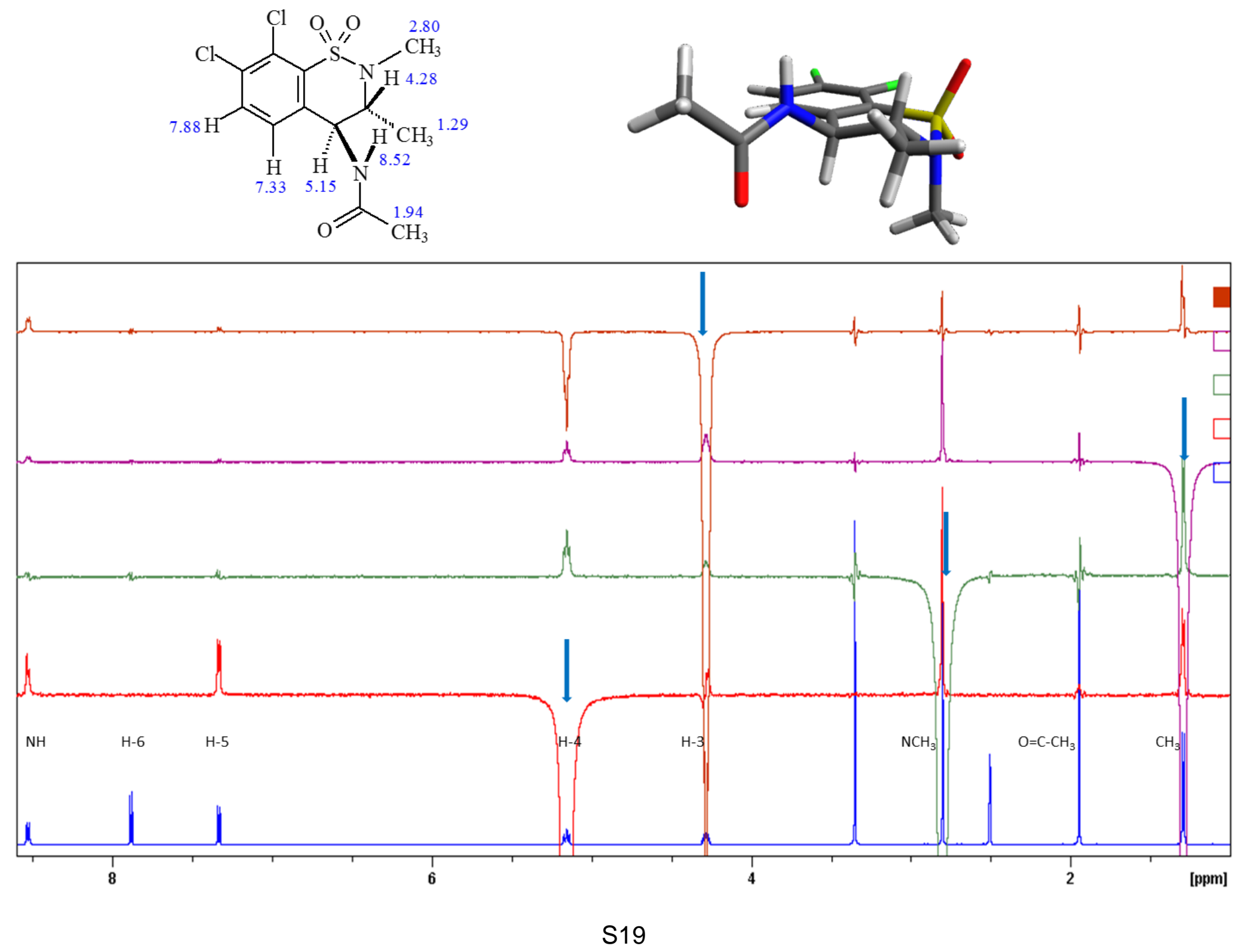
In the $85 \% / 15 \%$ mixture of $\mathbf{3} \mathbf{b}$ diastereomers, identification of the ${ }^{1} \mathrm{H}$ signals of the minor $\mathbf{c i s}-\mathbf{3 b}$ by selTOCSY and selNOE on $\delta \mathrm{NH}: 8.28 \mathrm{ppm}$, stereostructure supported also with DFT geometry optimization
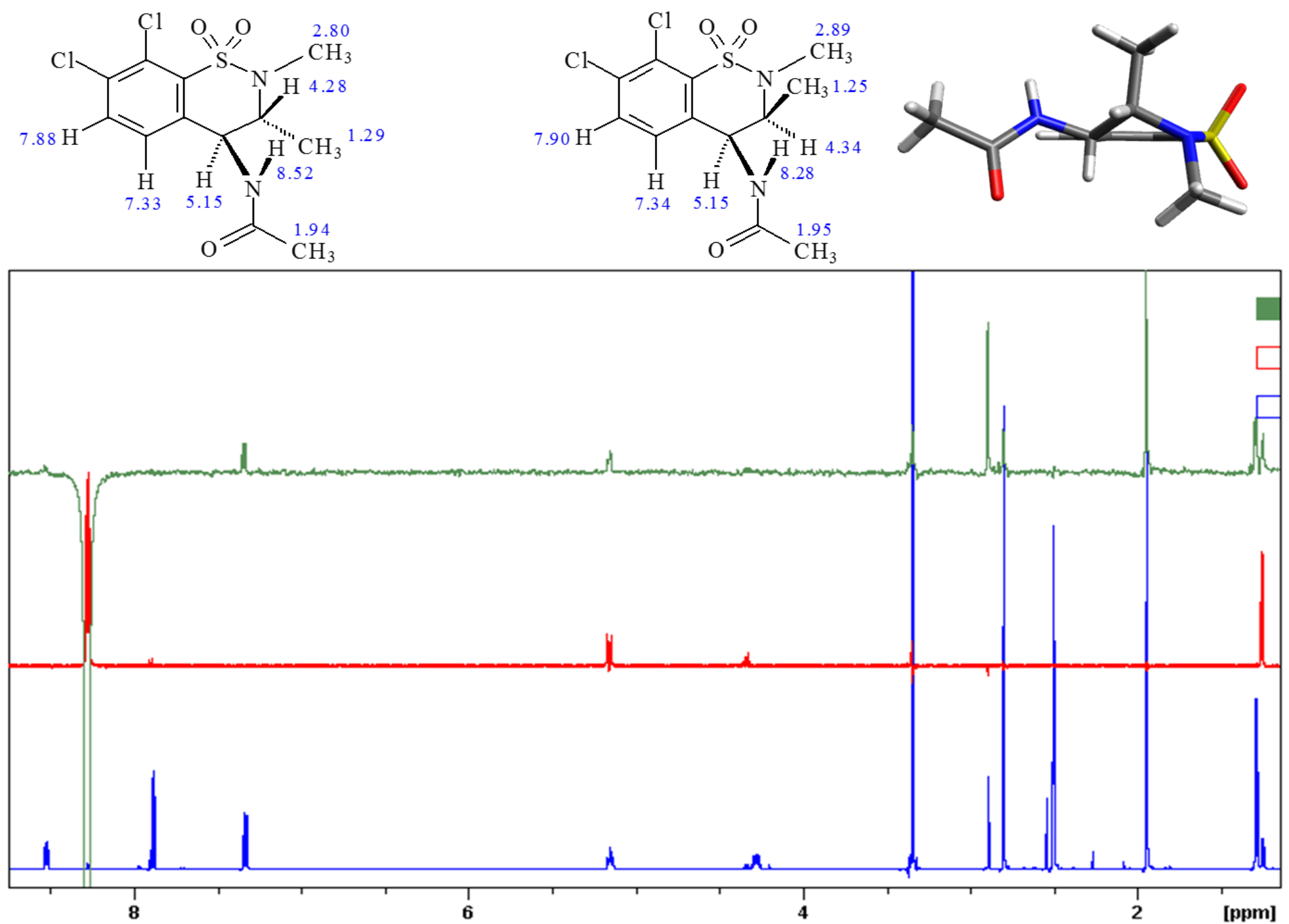
Investigation of the $\mathrm{H}-4$ and $\mathrm{H}-3$ signals detected in the $85 \% / 15 \%$ mixture of $\mathbf{3 b}$ diastereomers
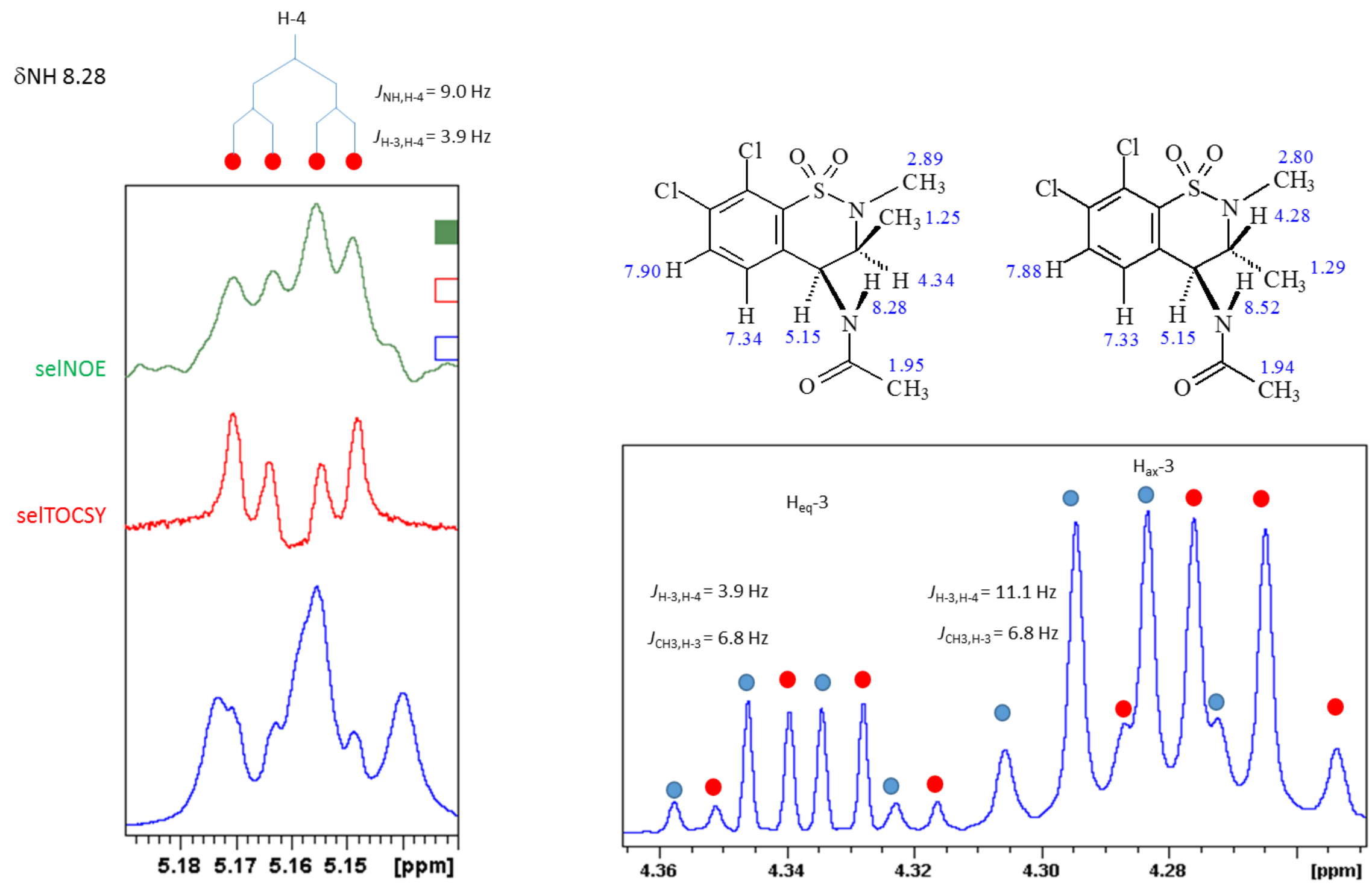
In the $85 \% / 15 \%$ mixture of $\mathbf{3 b}$ isomers, identification of the ${ }^{13} \mathrm{C}$ signals of the minor cis-3b by DEPTQ and edHSQC
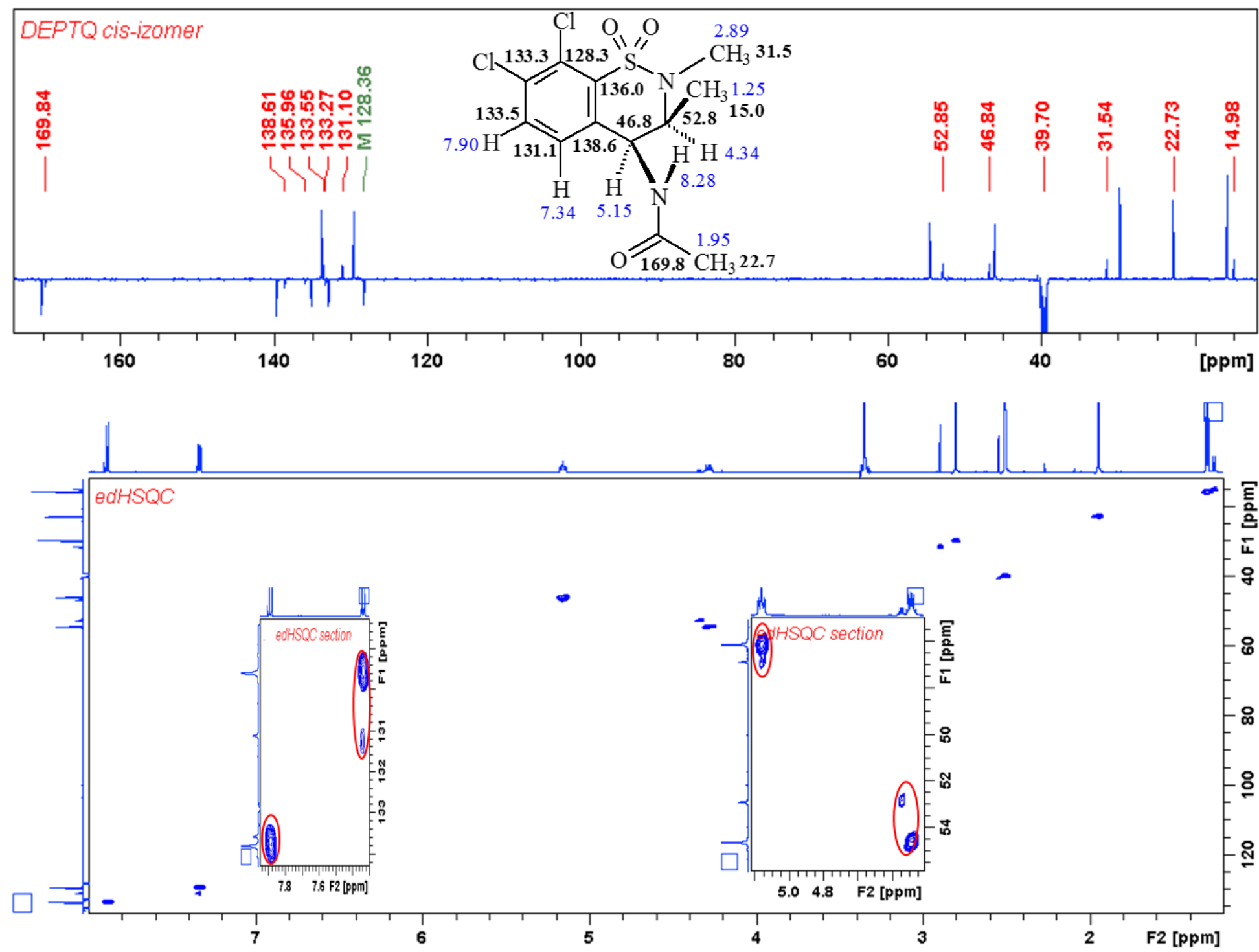
Reaction mixture of $\mathbf{1 a}$ and 1 equiv $\boldsymbol{t}$-BuOK in [D6]DMSO: compound $\mathrm{II}^{-}$and $\boldsymbol{t}$-BuO- ${ }^{-}{ }^{1} \mathrm{H}$ NMR and DEPTQ
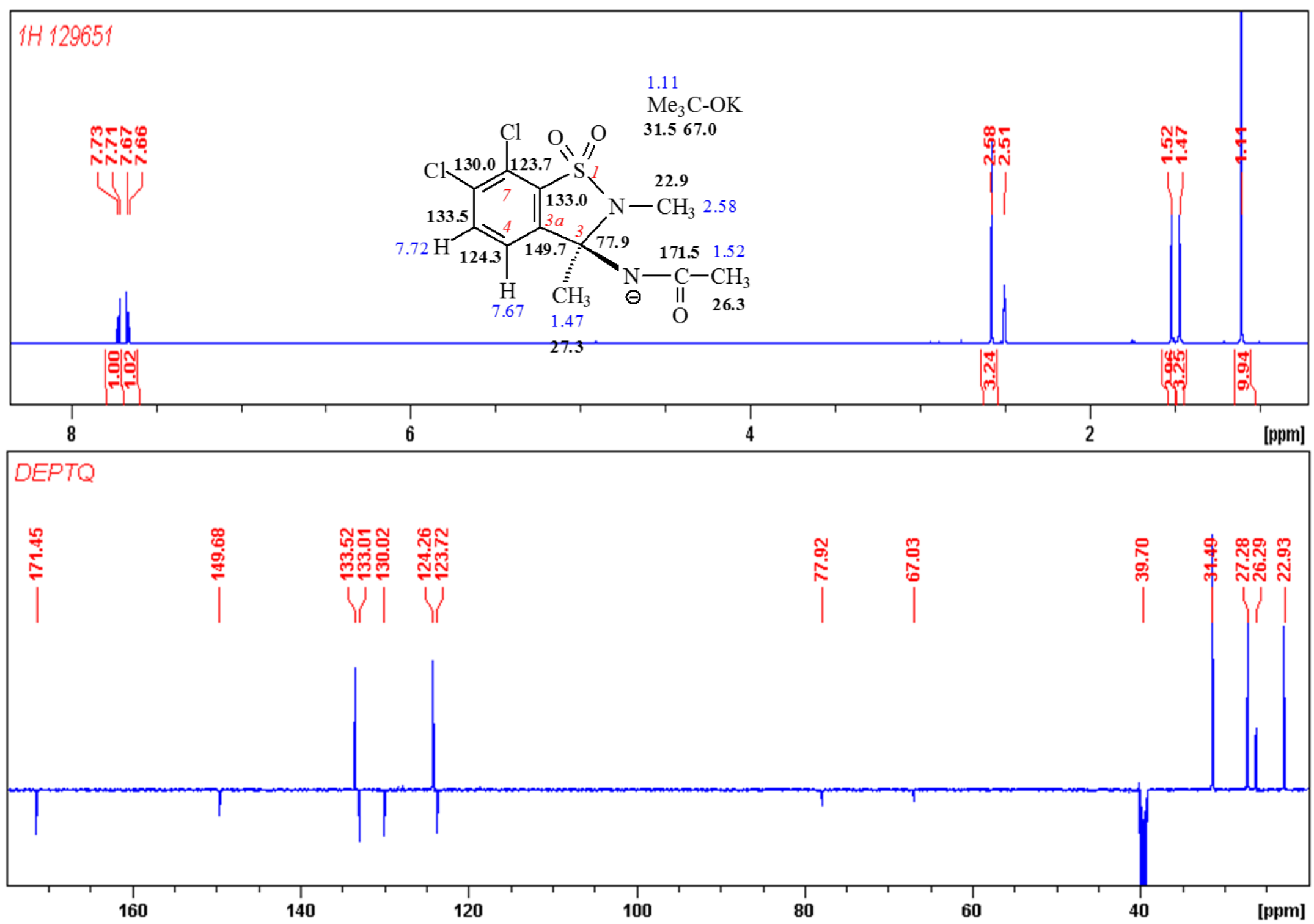
Reaction mixture of $\mathbf{1 a}$ and 1 equiv $\boldsymbol{t}$-BuOK in [D6]DMSO: compound $\mathrm{II}^{-}$and $\boldsymbol{t}-\mathrm{BuO}^{-}, \mathrm{HSQC}$ and $\mathrm{HMBC}$
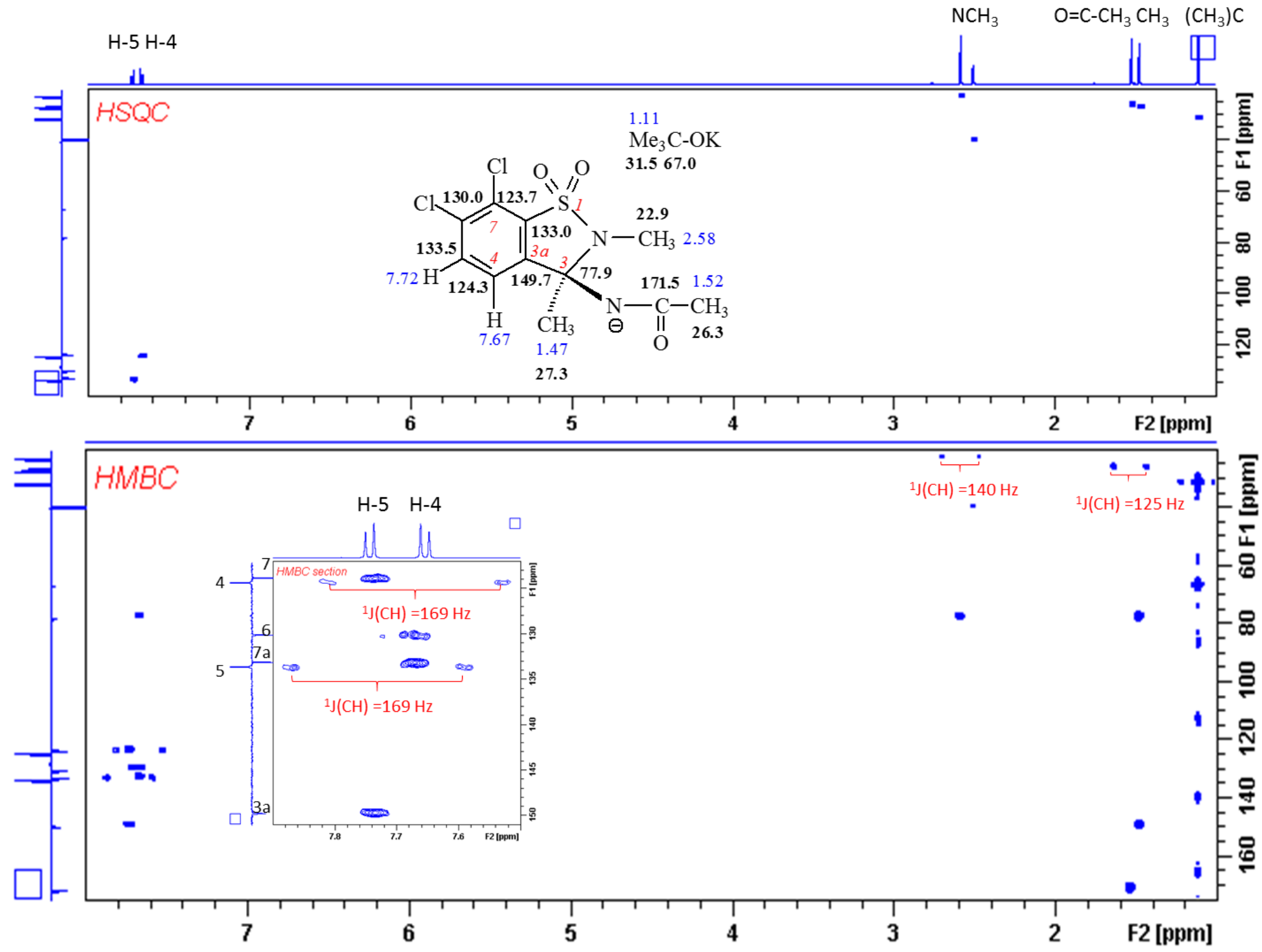
Reaction mixture of $\mathbf{1 a}$ and 2 equiv $\boldsymbol{t}$-BuOK in [D6]DMSO: compound $\mathrm{II}^{-}$, IV ${ }^{2-}$ and $\boldsymbol{t}$-BuO-, HSQC and HMBC
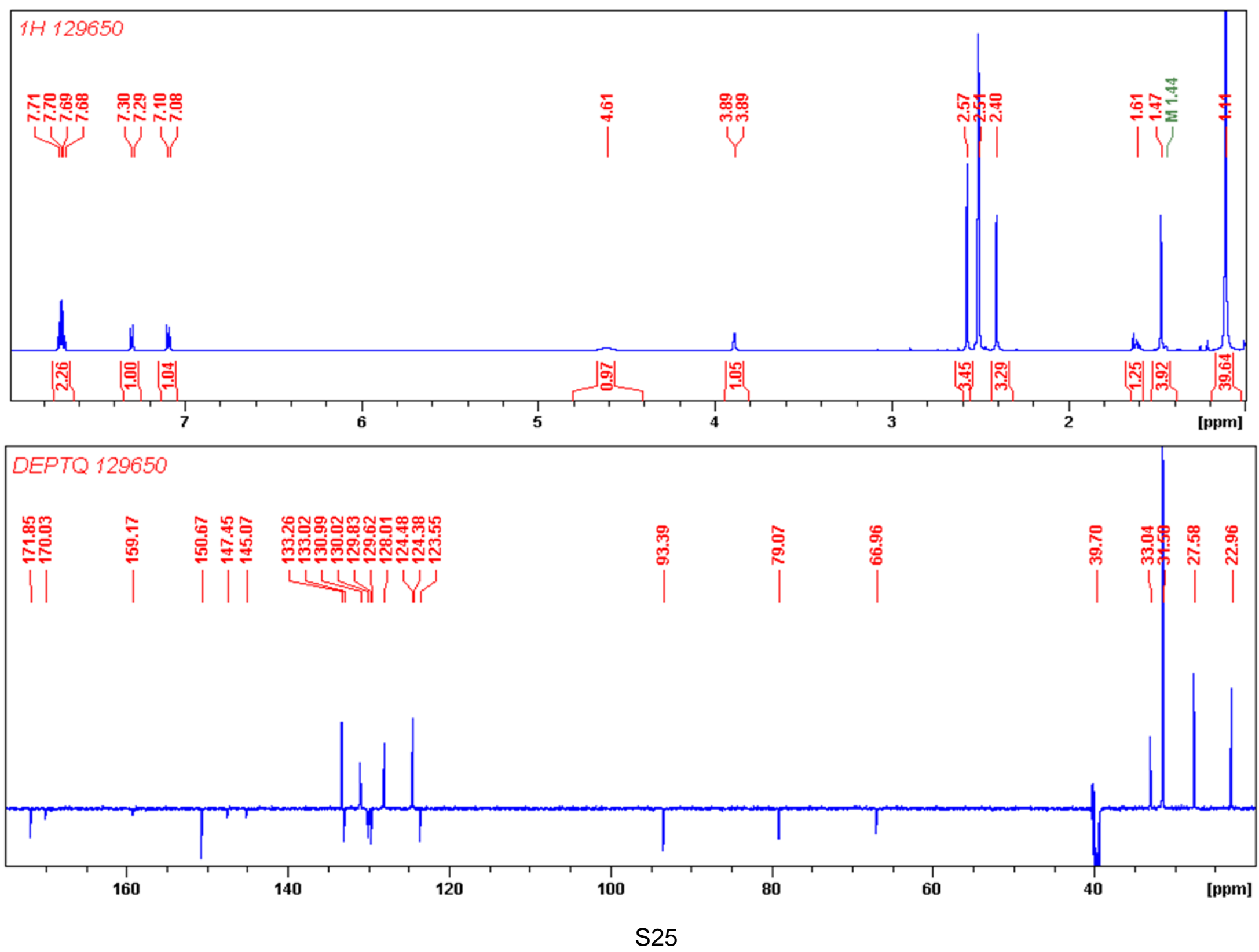
Reaction mixture of $\mathbf{1 a}$ and 6 equiv $\boldsymbol{t}$-BuOK in [D6]DMSO: compound $\mathbf{I V}^{2-}$ and $\boldsymbol{t}$-BuO-,${ }^{1} \mathrm{H}$, DEPTQ and ${ }^{13} \mathrm{C}$ NMR

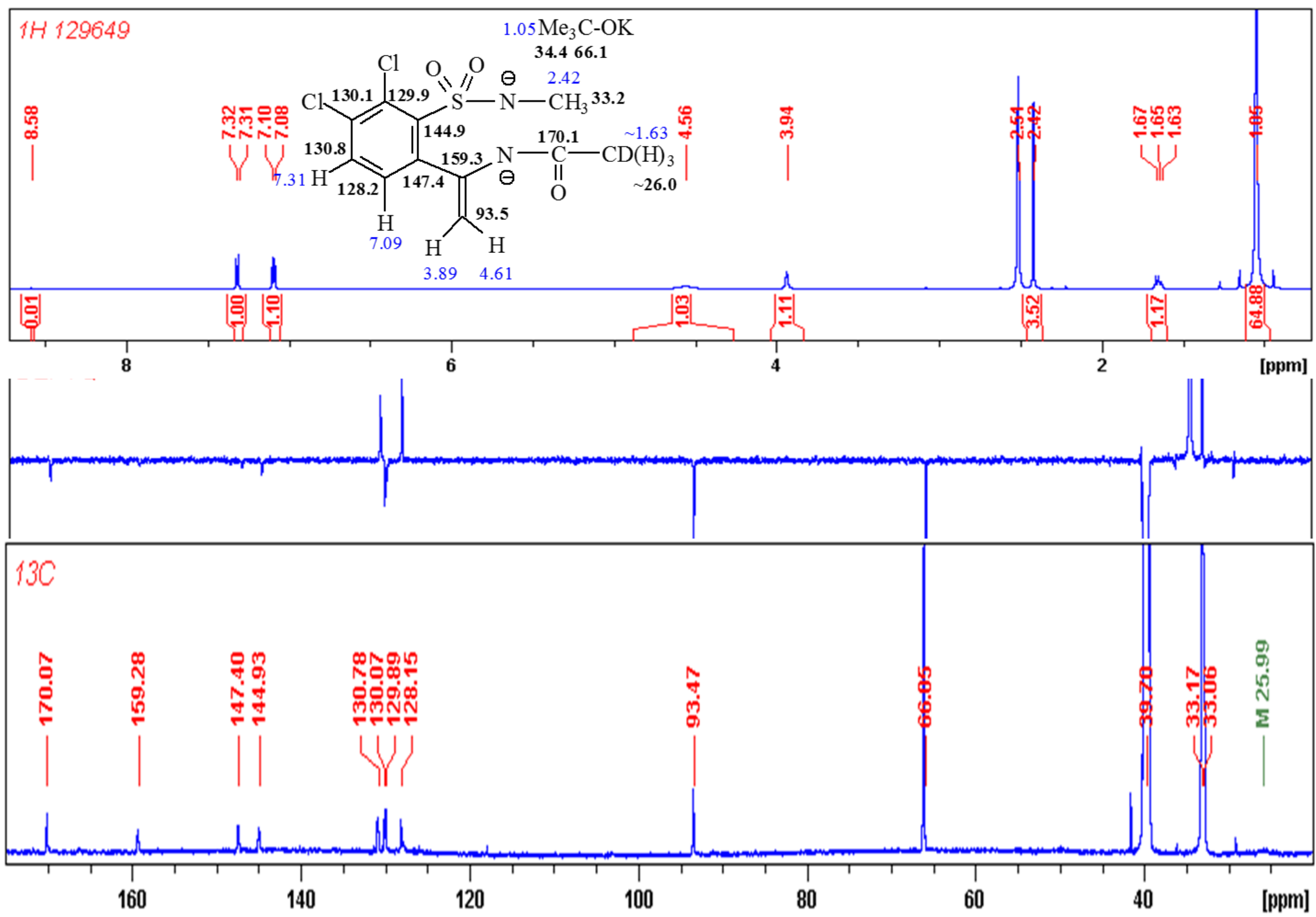


Reaction mixture of $\mathbf{1 a}$ and 6 equiv $\boldsymbol{t}$-BuOK in [D6]DMSO]DMSO: compound IV $^{2-}$ and $\boldsymbol{t}$-BuO-, HSQC and HMBC
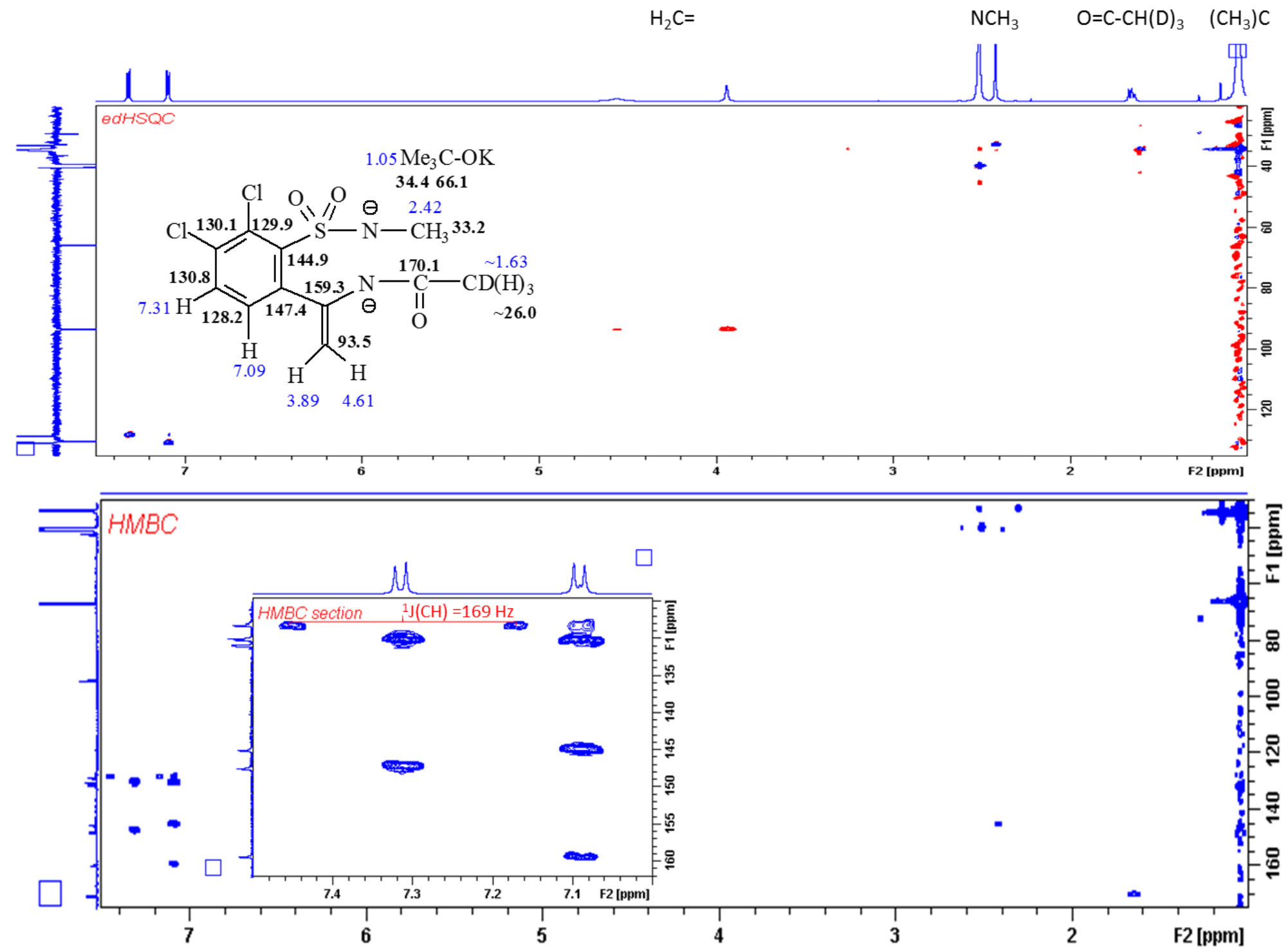
Compound [D]3a, ${ }^{1} \mathrm{H}, \mathrm{HSQC}$ and ${ }^{13} \mathrm{C}$ NMR

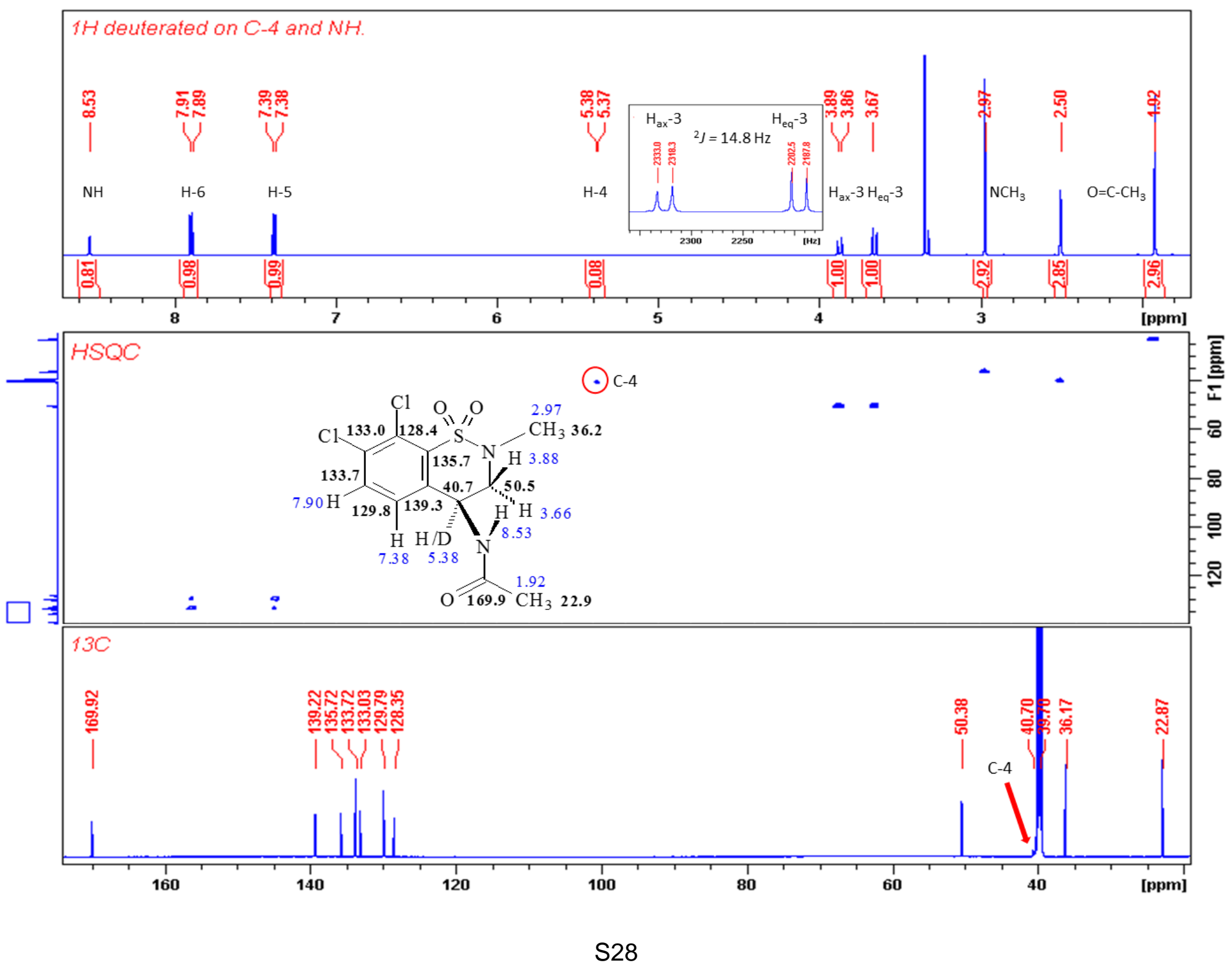




\section{NMR spectra}

Compound 1c, ${ }^{1} \mathrm{H}$ and ${ }^{13} \mathrm{C}$ NMR $\left(600 / 150 \mathrm{MHz},\left[\mathrm{D}_{6}\right] \mathrm{DMSO}\right)$<smiles>CCN1C(C)c2ccc(Cl)c(Cl)c2S(=O)(=O)N1C(C)=O</smiles>

V
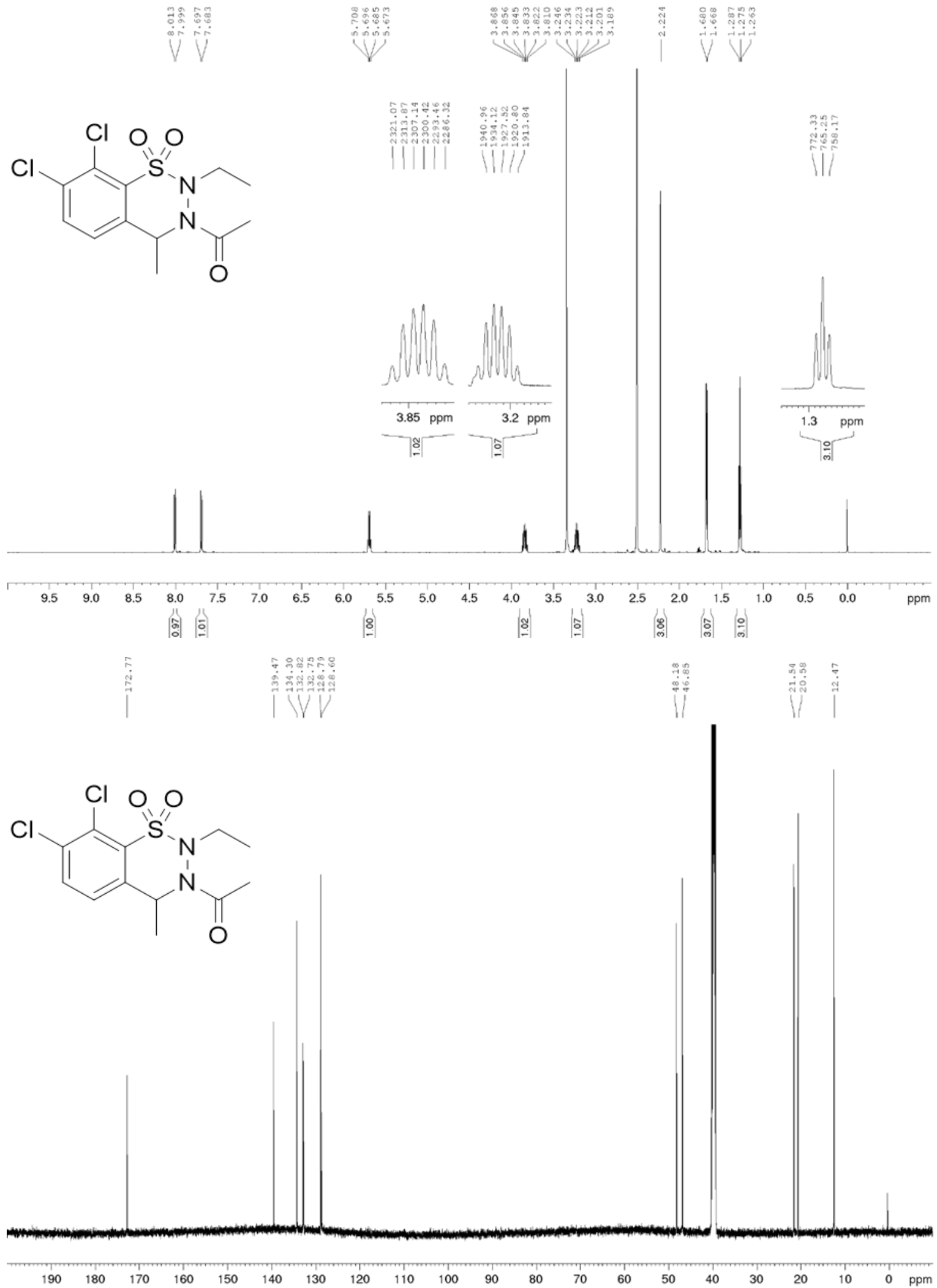
Compound 1d, ${ }^{1} \mathrm{H}$ and ${ }^{13} \mathrm{C}$ NMR $\left(600 / 150 \mathrm{MHz},\left[\mathrm{D}_{6}\right] \mathrm{DMSO}\right)$

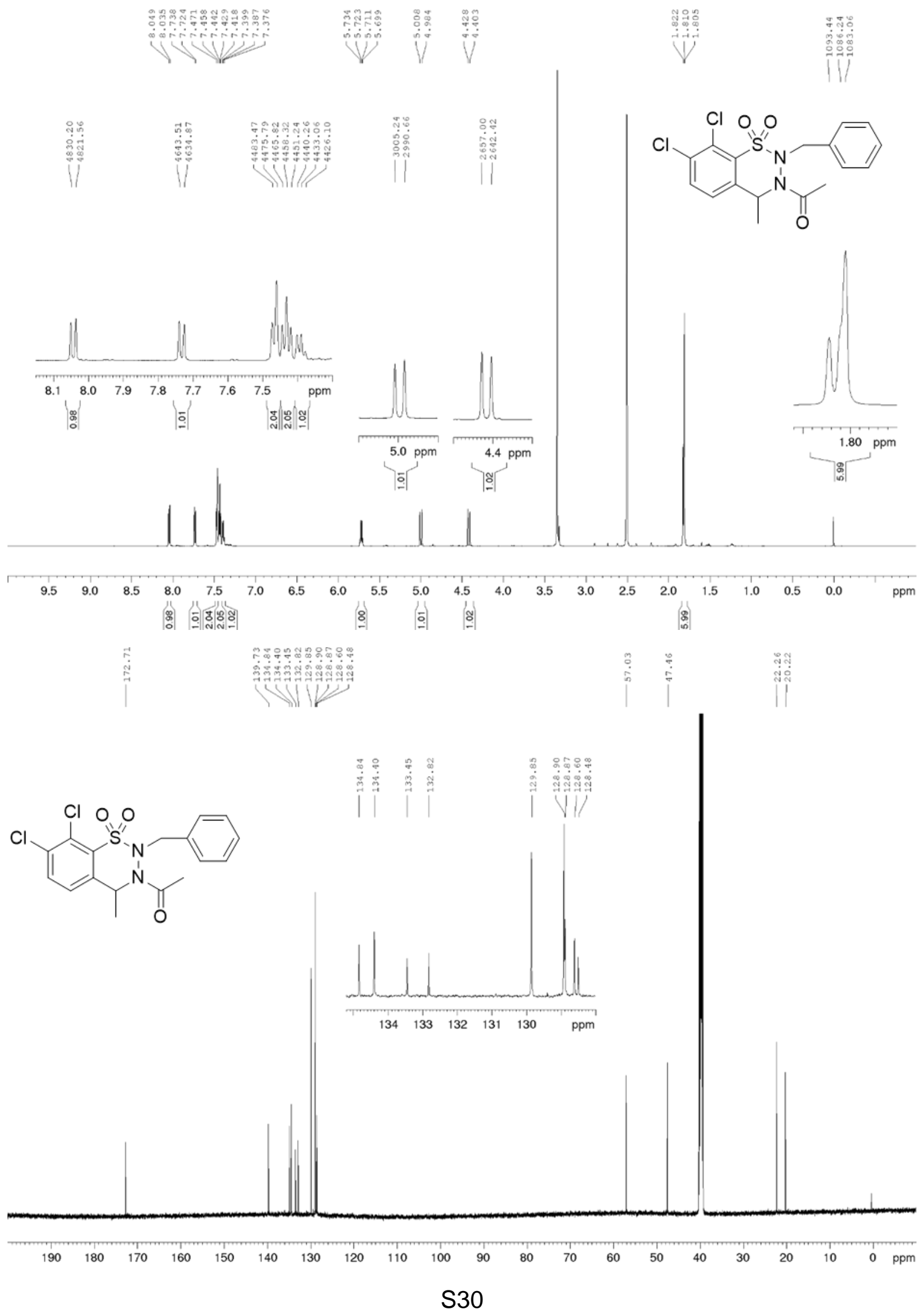


Compound 7b, ${ }^{1} \mathrm{H}$ and ${ }^{13} \mathrm{C}$ NMR $\left(600 / 150 \mathrm{MHz}, \mathrm{CDCl}_{3}\right)$

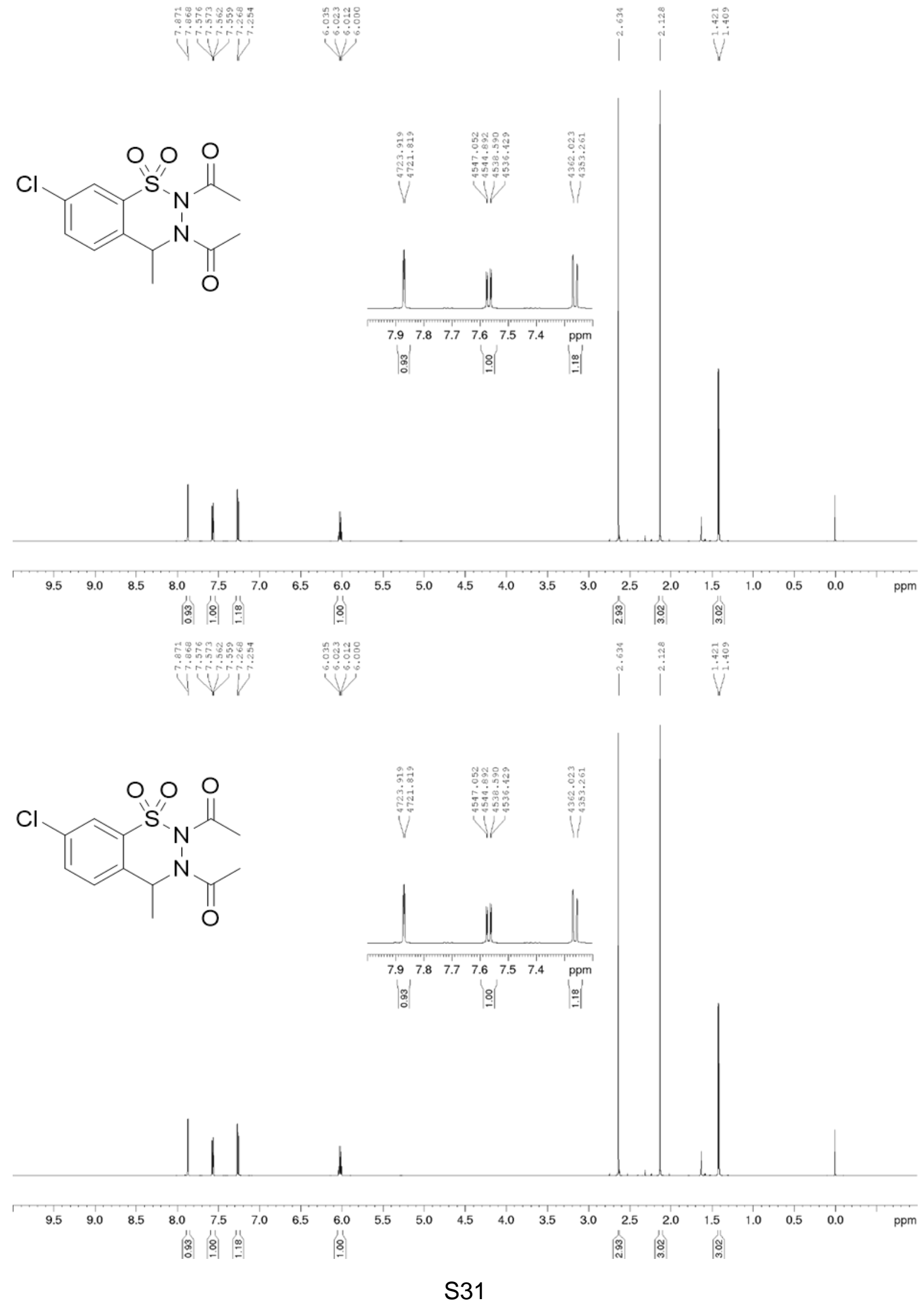


Compound 7c, ${ }^{1} \mathrm{H}$ and ${ }^{13} \mathrm{C}$ NMR $\left(600 / 150 \mathrm{MHz}, \mathrm{CDCl}_{3}\right)$
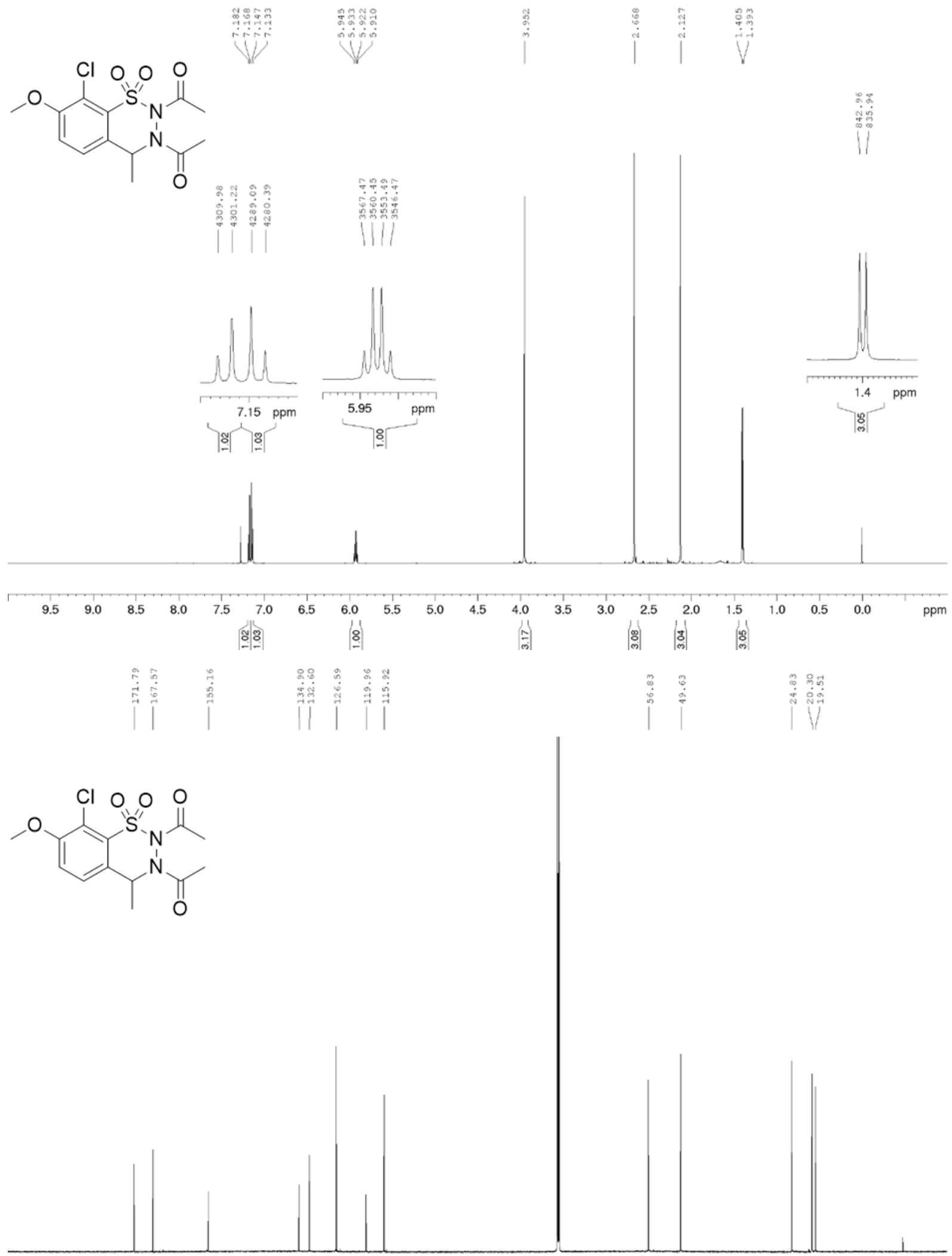

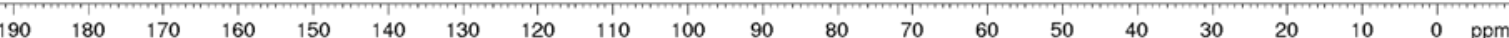
S32 
Compound $7 d,{ }^{1} \mathrm{H}$ and ${ }^{13} \mathrm{C} \mathrm{NMR}\left(600 / 150 \mathrm{MHz}, \mathrm{CDCl}_{3}\right)$

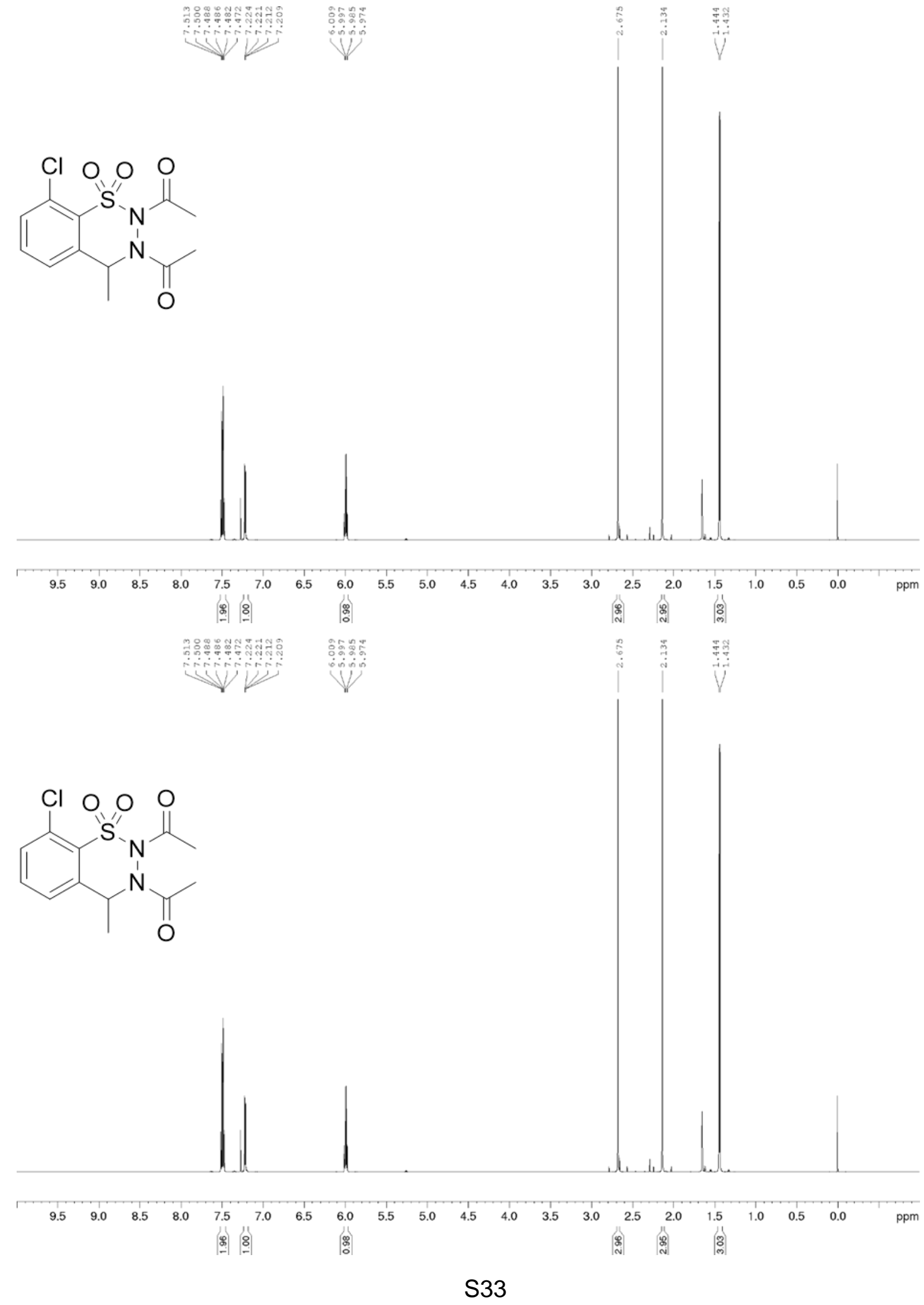


Compound $7 e,{ }^{1} \mathrm{H}$ and ${ }^{13} \mathrm{C} \mathrm{NMR}\left(600 / 150 \mathrm{MHz}, \mathrm{CDCl}_{3}\right)$

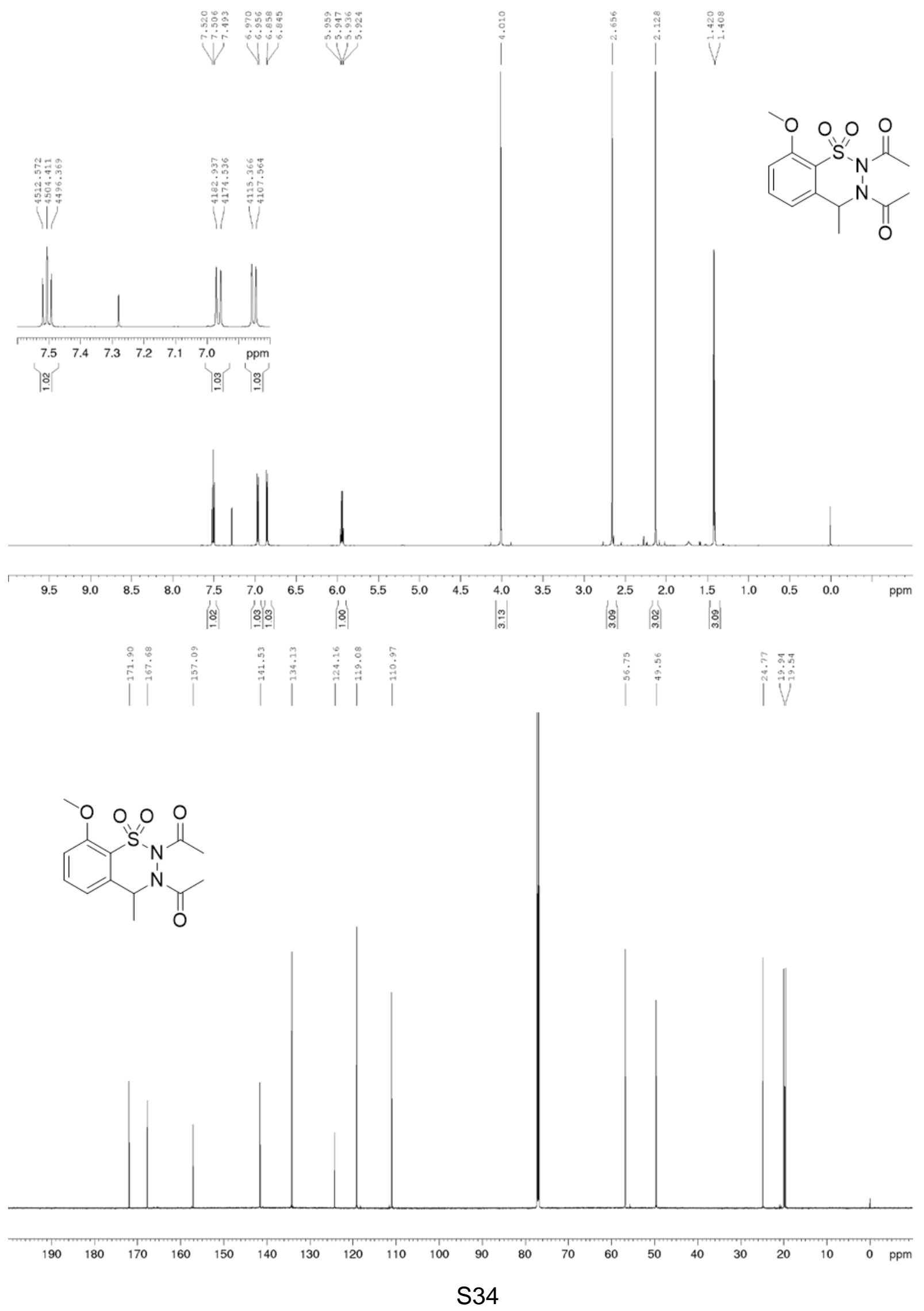


Compound 7f, ${ }^{1} \mathrm{H}$ and ${ }^{13} \mathrm{C}$ NMR $\left(600 / 150 \mathrm{MHz}, \mathrm{CDCl}_{3}\right)$

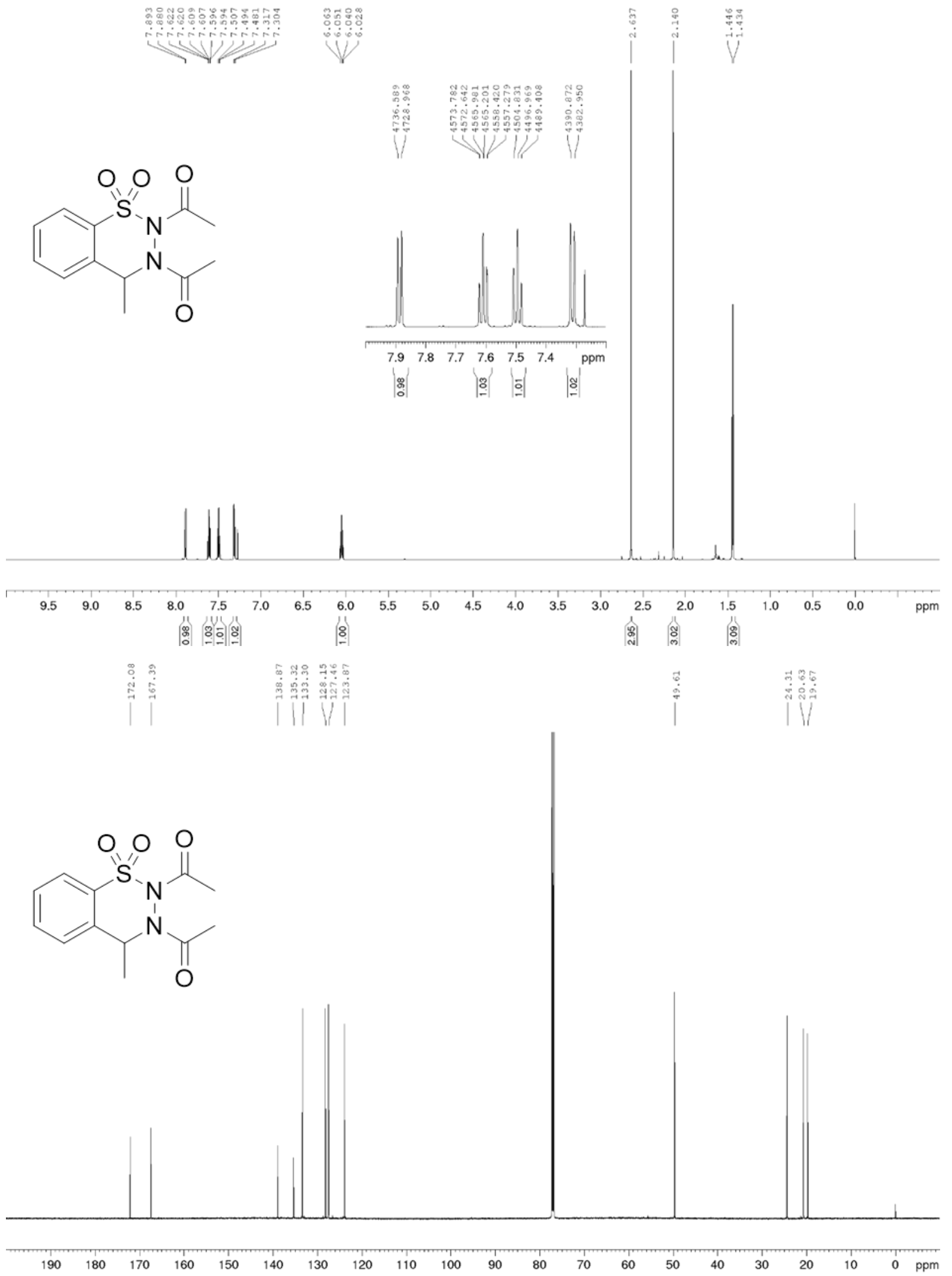


Compound 1k, ${ }^{1} \mathrm{H}$ and ${ }^{13} \mathrm{C}$ NMR $\left(600 / 150 \mathrm{MHz},\left[\mathrm{D}_{6}\right.\right.$ ]DMSO)
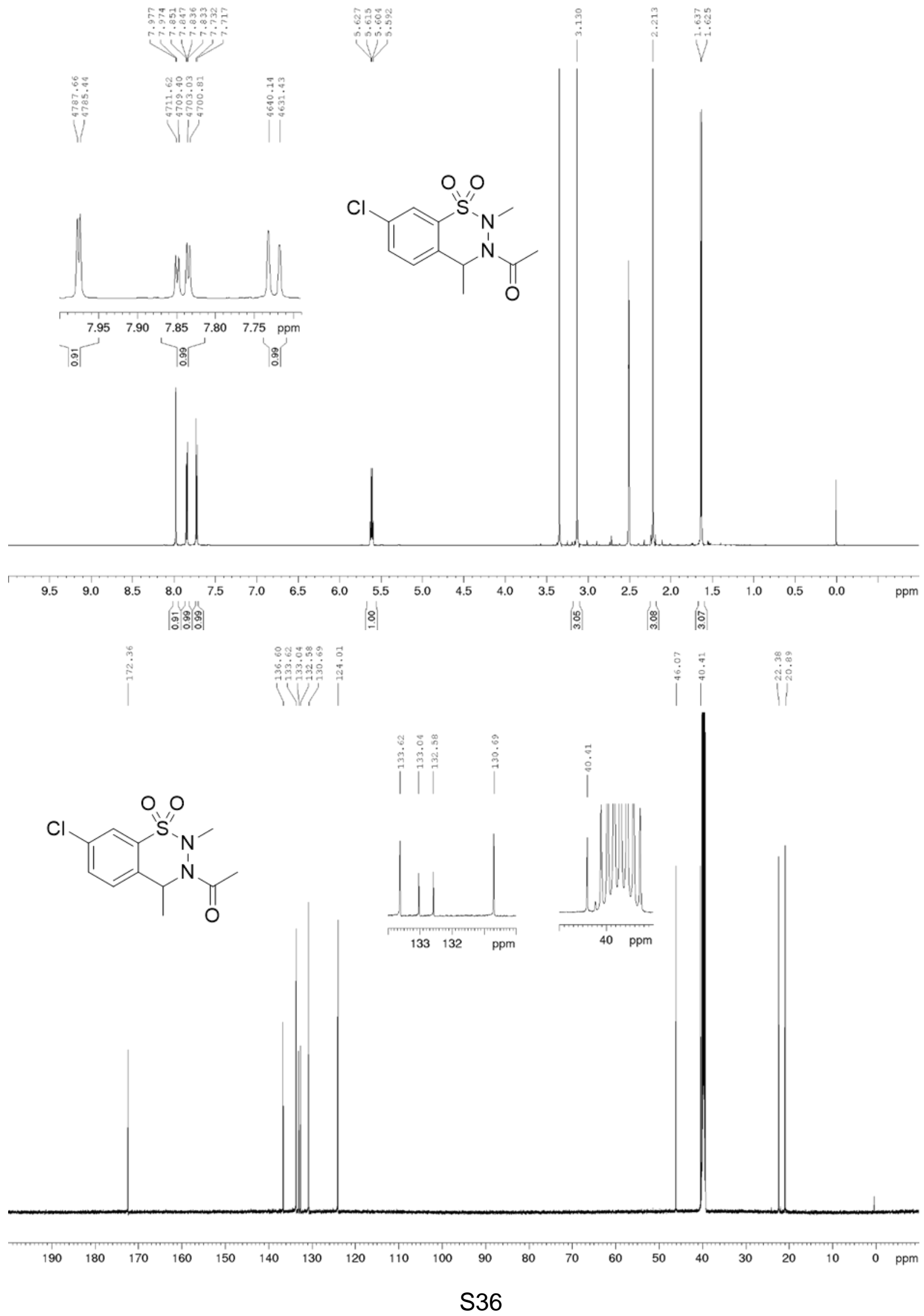
Compound 11, ${ }^{1} \mathrm{H}$ and ${ }^{13} \mathrm{C}$ NMR $\left(400 / 100 \mathrm{MHz},\left[\mathrm{D}_{6}\right] \mathrm{DMSO}\right)$

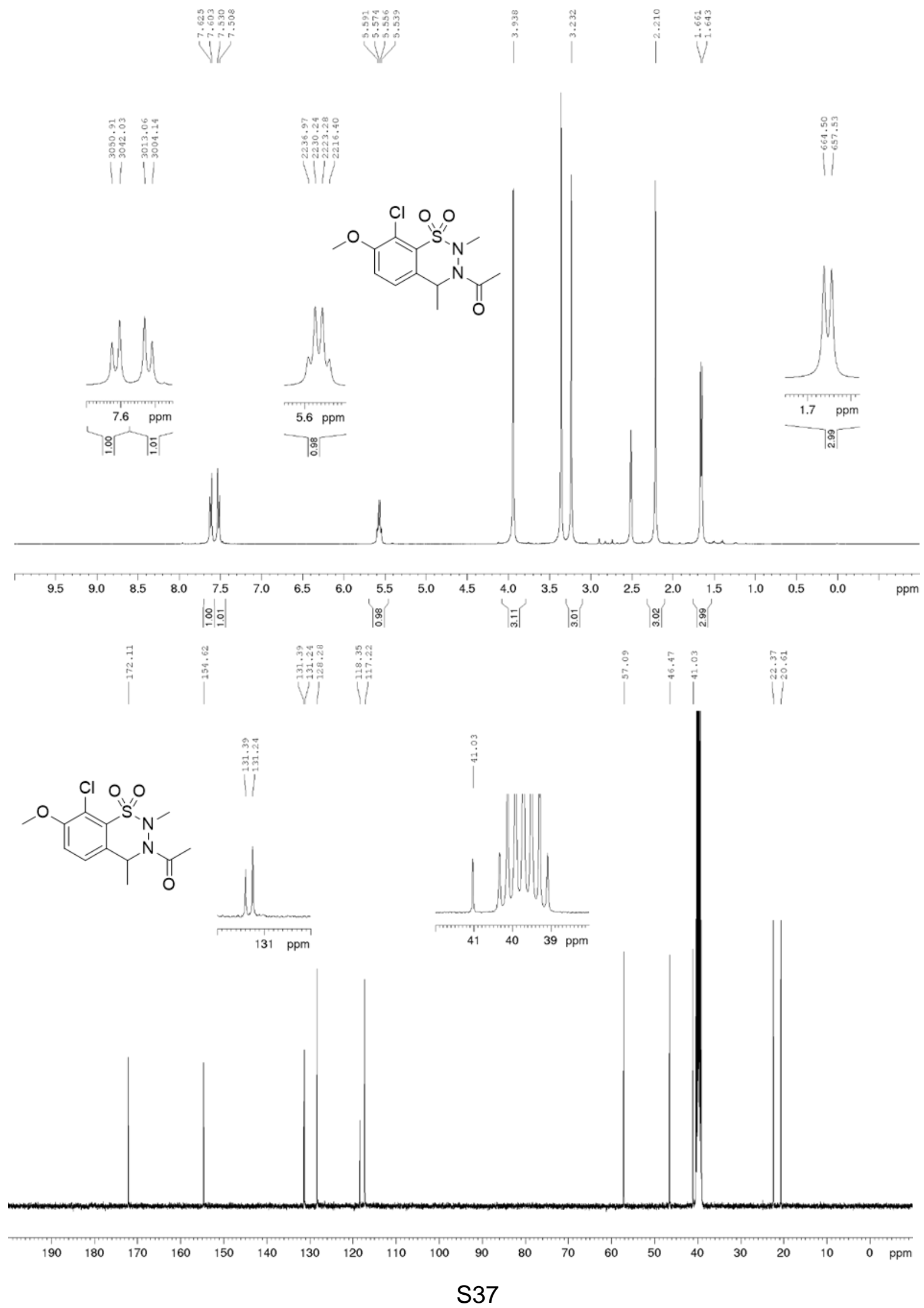


Compound 1m, ${ }^{1} \mathrm{H}$ and ${ }^{13} \mathrm{C}$ NMR (600/150 MHz, [D6]DMSO)
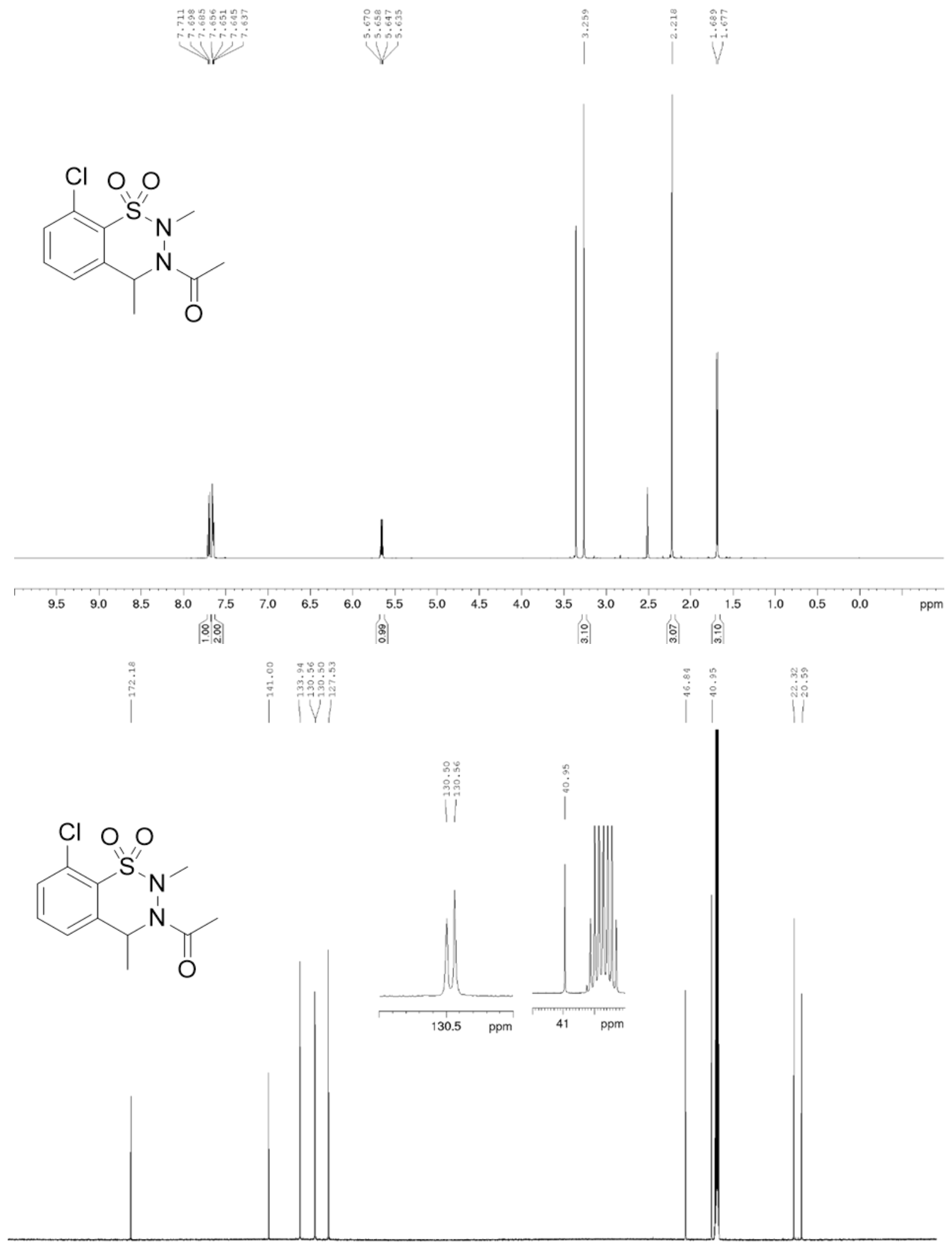

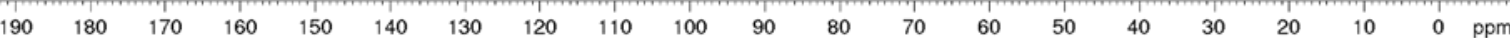
S38 
Compound 1n, ${ }^{1} \mathrm{H}$ and ${ }^{13} \mathrm{C}$ NMR $\left(400 / 100 \mathrm{MHz},\left[\mathrm{D}_{6}\right] \mathrm{DMSO}\right)$

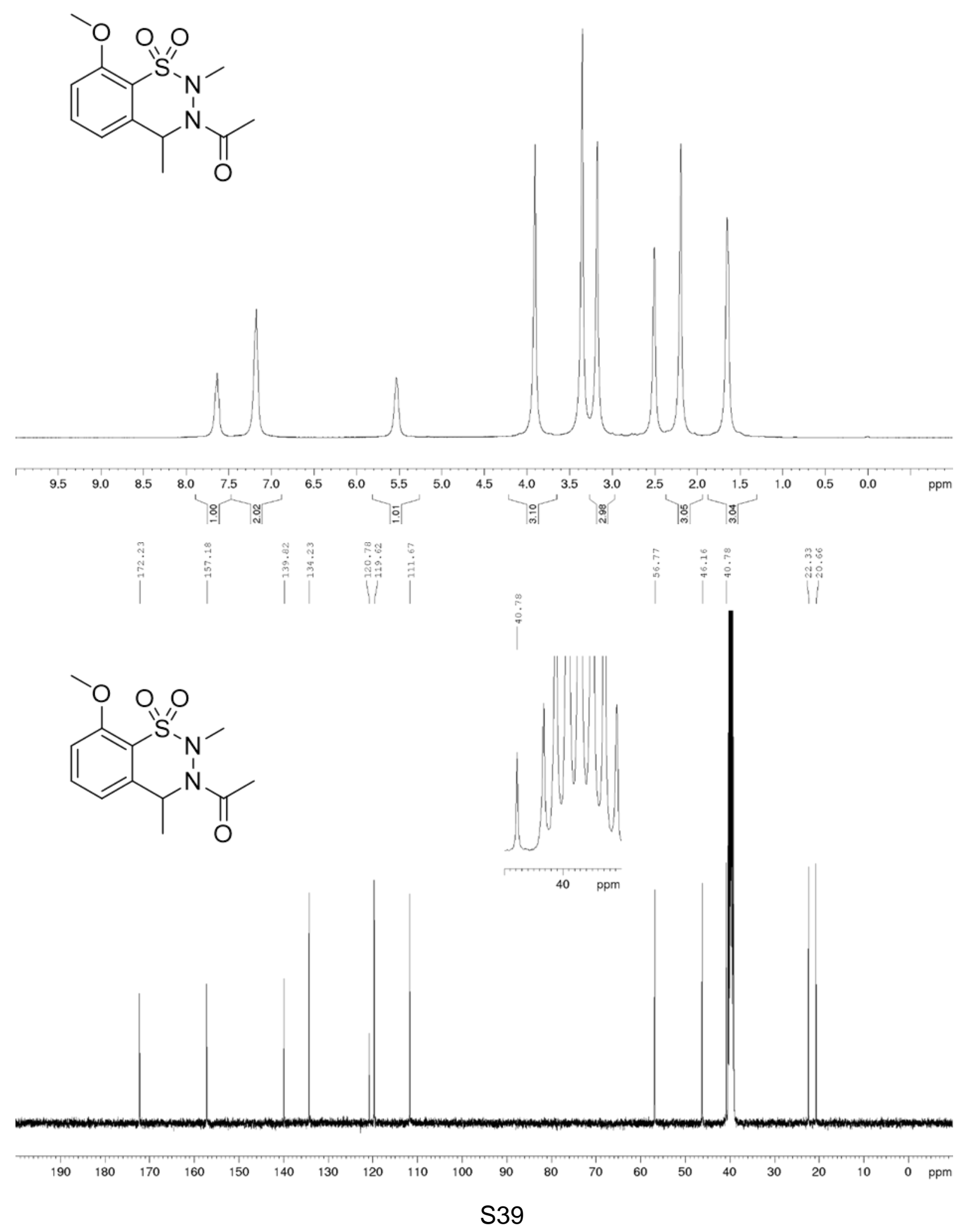


Compound 1o, ${ }^{1} \mathrm{H}$ and ${ }^{13} \mathrm{C}$ NMR $\left(600 / 150 \mathrm{MHz},\left[\mathrm{D}_{6}\right] \mathrm{DMSO}\right)$

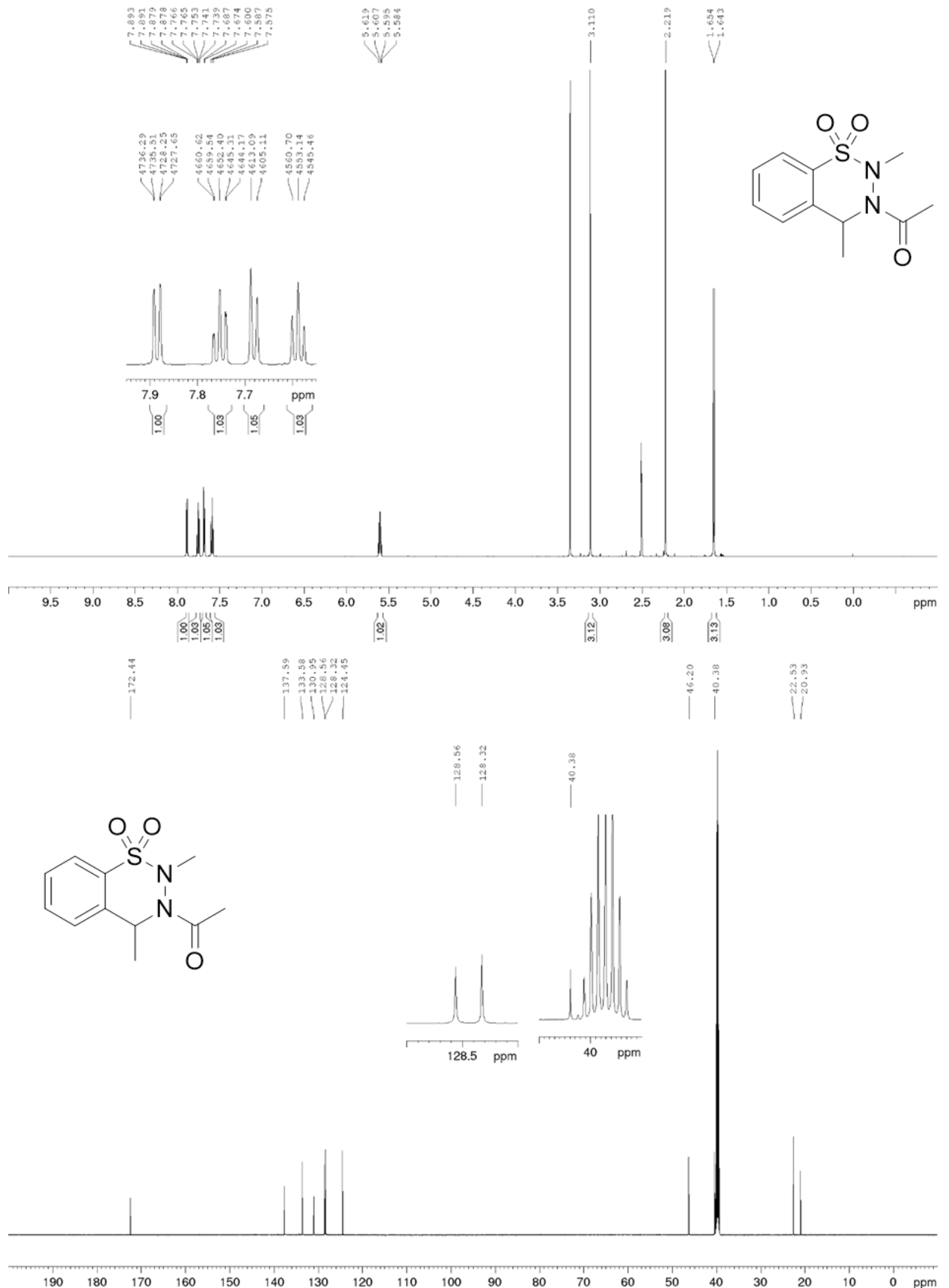


Compound 1e, ${ }^{1} \mathrm{H}$ and ${ }^{13} \mathrm{C}$ NMR $\left(600 / 150 \mathrm{MHz},\left[\mathrm{D}_{6}\right.\right.$ ]DMSO)

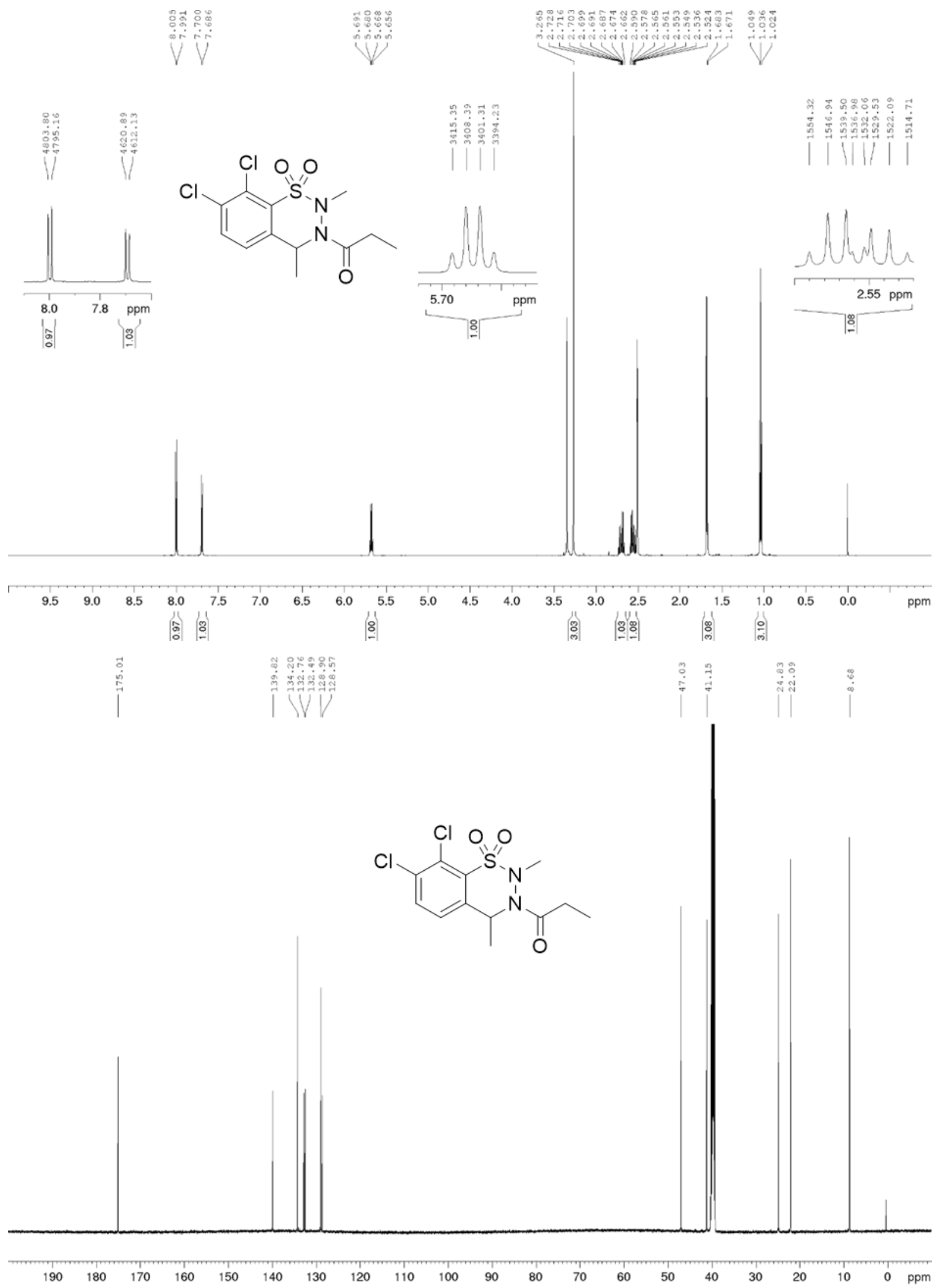


Compound 1f, ${ }^{1} \mathrm{H}$ and ${ }^{13} \mathrm{C}$ NMR (600/150 MHz, [D6]DMSO)
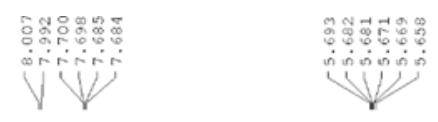

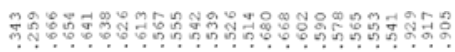

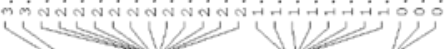<smiles>CCCC(=O)N1C(C)c2ccc(Cl)c(Cl)c2S(=O)(=O)N1C</smiles>
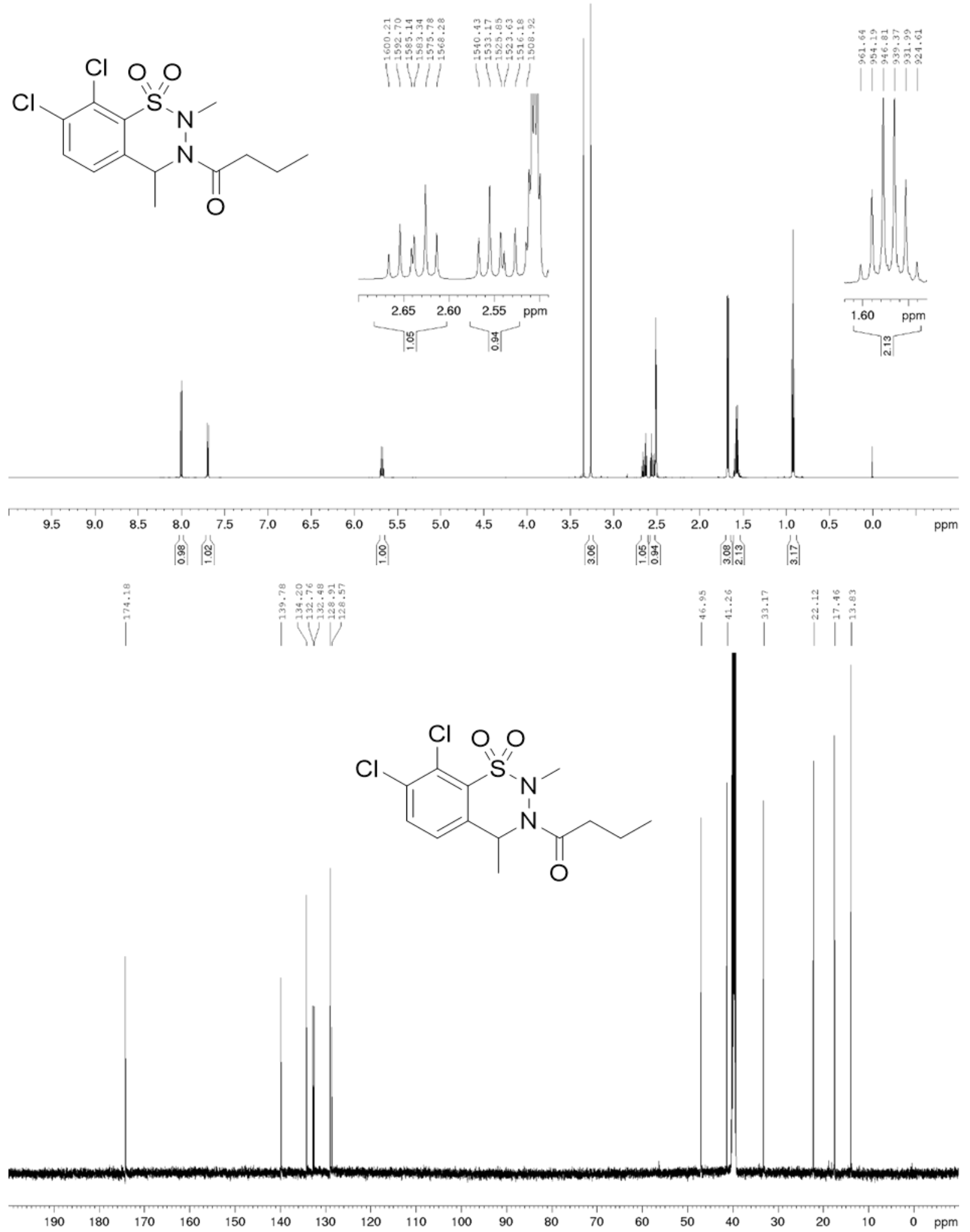
Compound 1g, ${ }^{1} \mathrm{H}$ and ${ }^{13} \mathrm{C}$ NMR $\left(600 / 150 \mathrm{MHz},\left[\mathrm{D}_{6}\right.\right.$ ]DMSO)

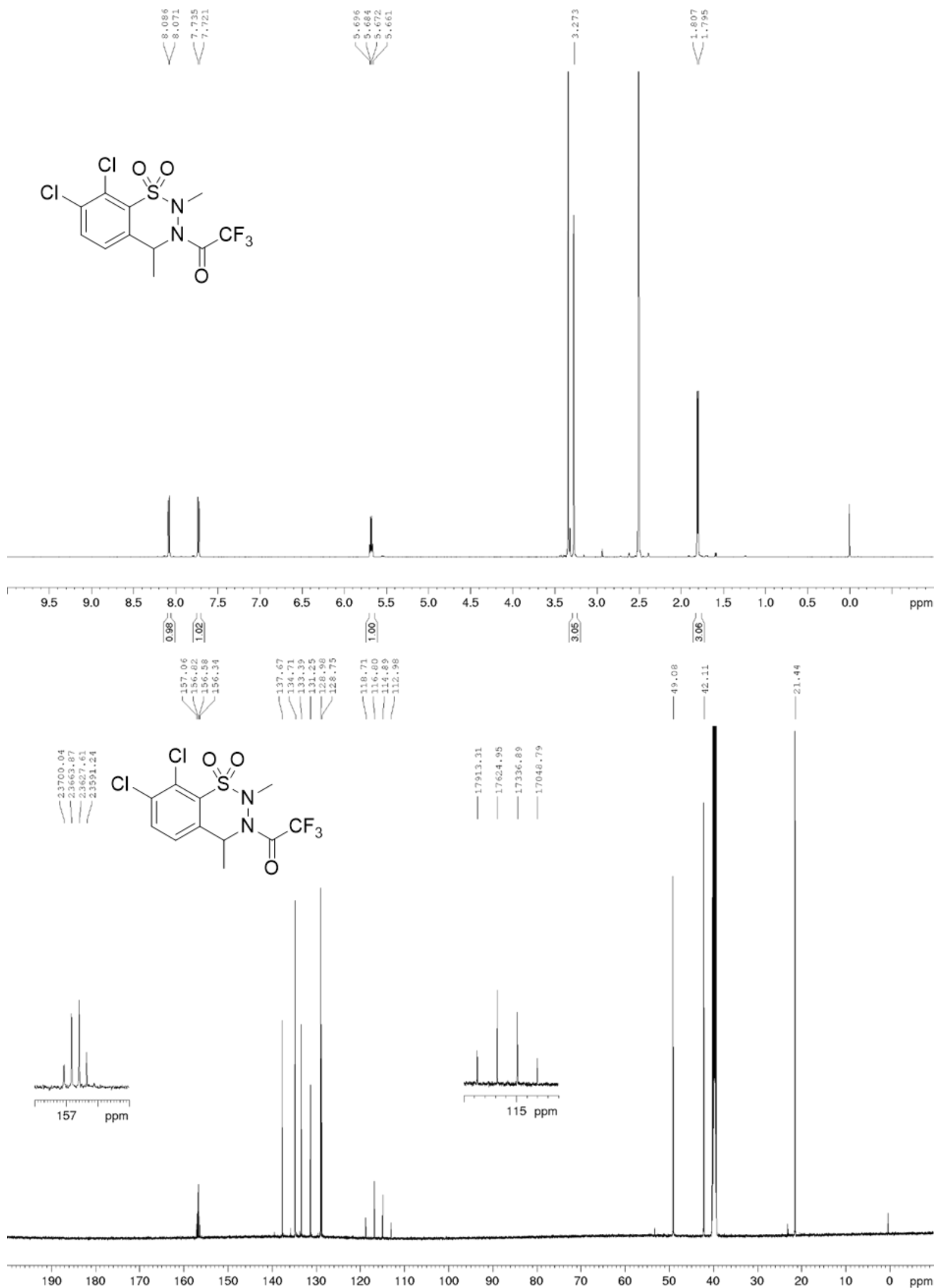

S43 
Compound Th, ${ }^{1} \mathrm{H}$ and ${ }^{13} \mathrm{C}$ NMR $\left(600 / 150 \mathrm{MHz}, \mathrm{CDCl}_{3}\right)$

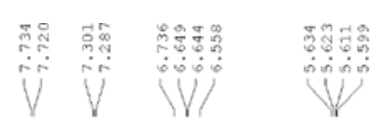

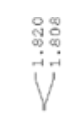
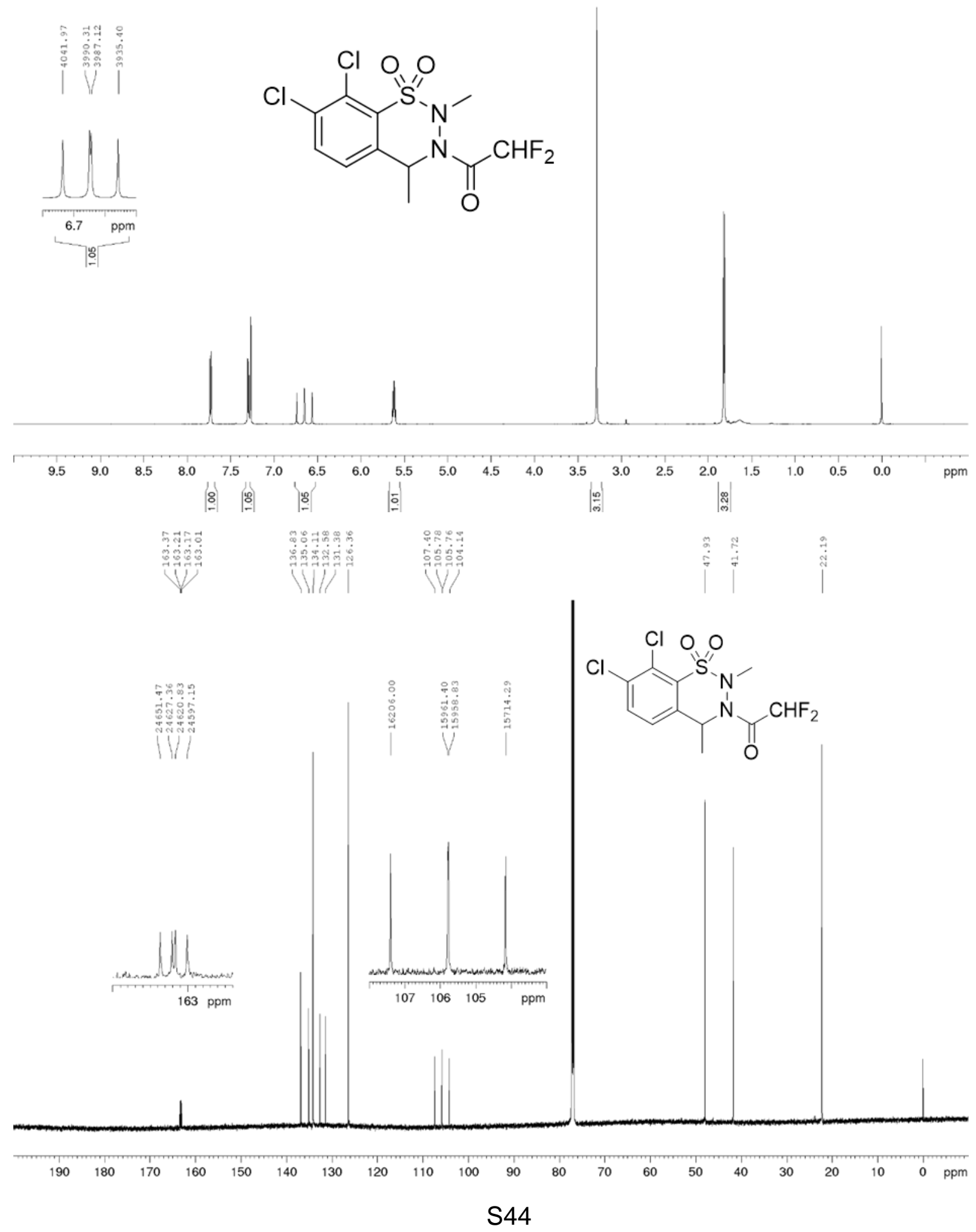
Compound 1p, ${ }^{1} \mathrm{H}$ and ${ }^{13} \mathrm{C}$ NMR $\left(600 / 150 \mathrm{MHz}, \mathrm{CDCl}_{3}\right)$
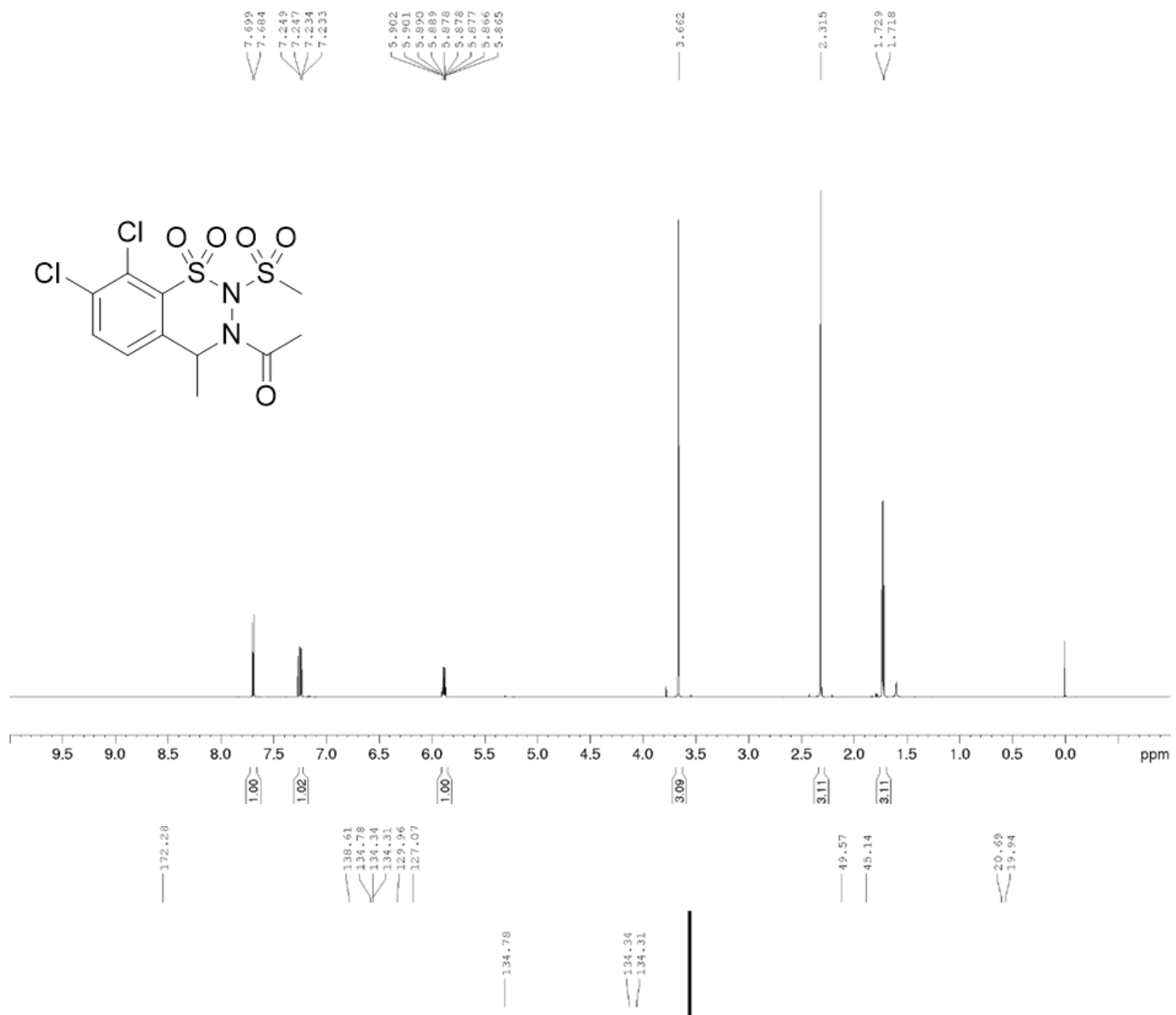<smiles>CC(=O)N1C(C)c2ccc(Cl)c(Cl)c2S(=O)(=O)N1S(C)(=O)=O</smiles>

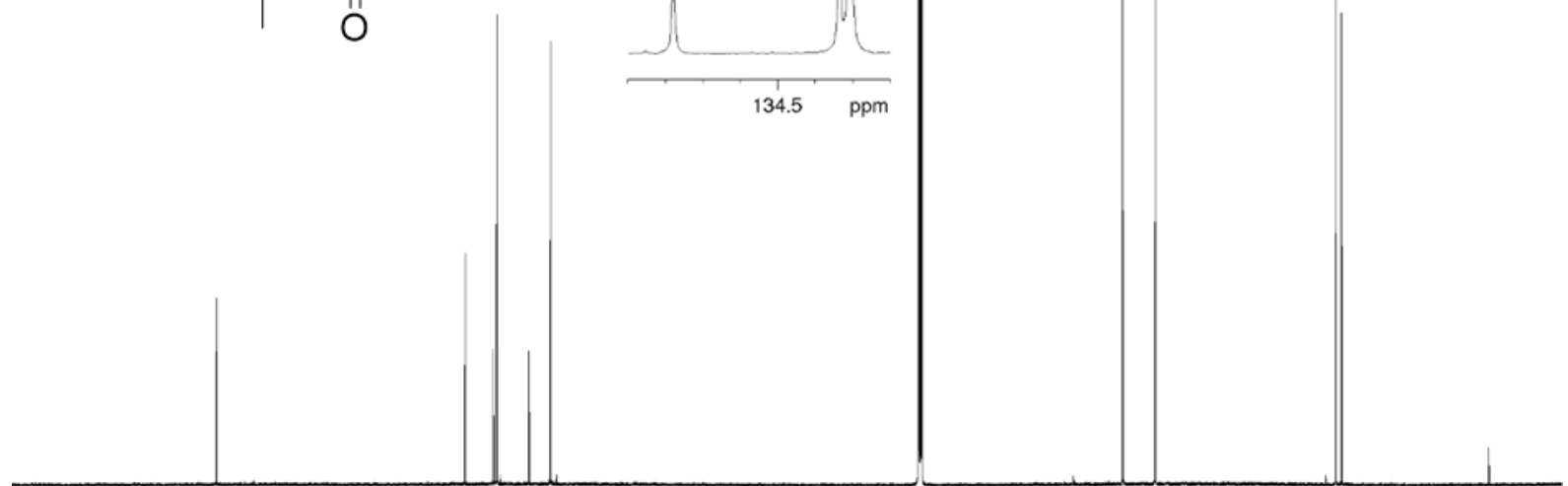

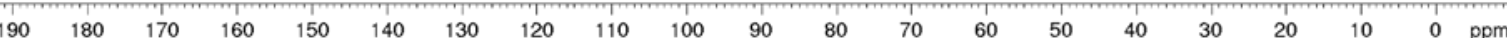


Compound 1q, ${ }^{1} \mathrm{H}$ and ${ }^{13} \mathrm{C}$ NMR $\left(600 / 150 \mathrm{MHz}, \mathrm{CDCl}_{3}\right)$
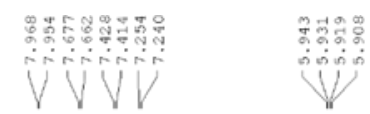

V1

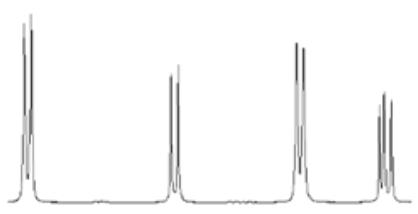<smiles>CC(=O)N1C(C)c2ccc(Cl)c(Cl)c2S(=O)(=O)N1S(=O)(=O)c1ccc(C)cc1</smiles>
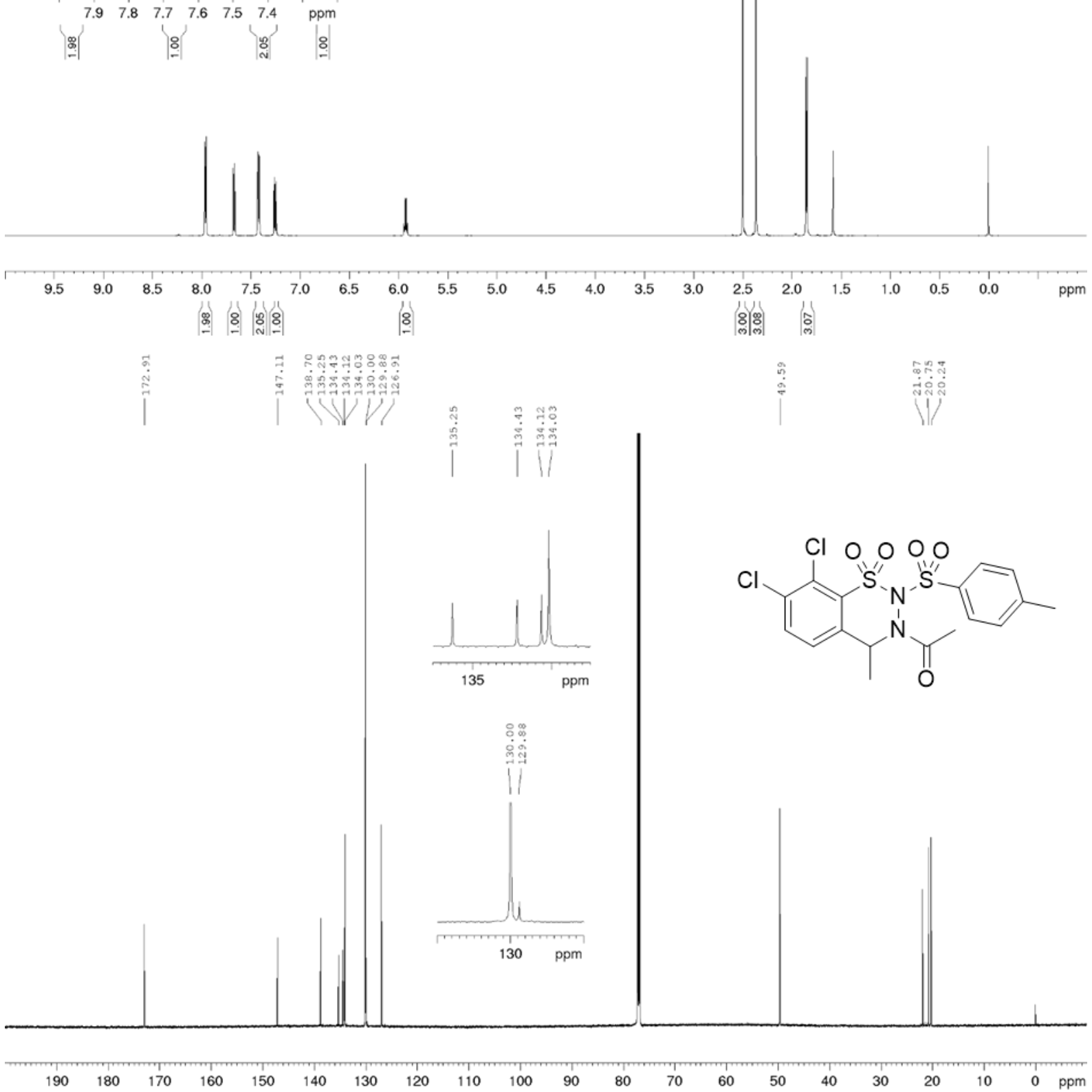
Compound 2a, ${ }^{1} \mathrm{H}$ and ${ }^{13} \mathrm{C}$ NMR $\left(600 / 150 \mathrm{MHz},\left[\mathrm{D}_{6}\right] \mathrm{DMSO}\right)$
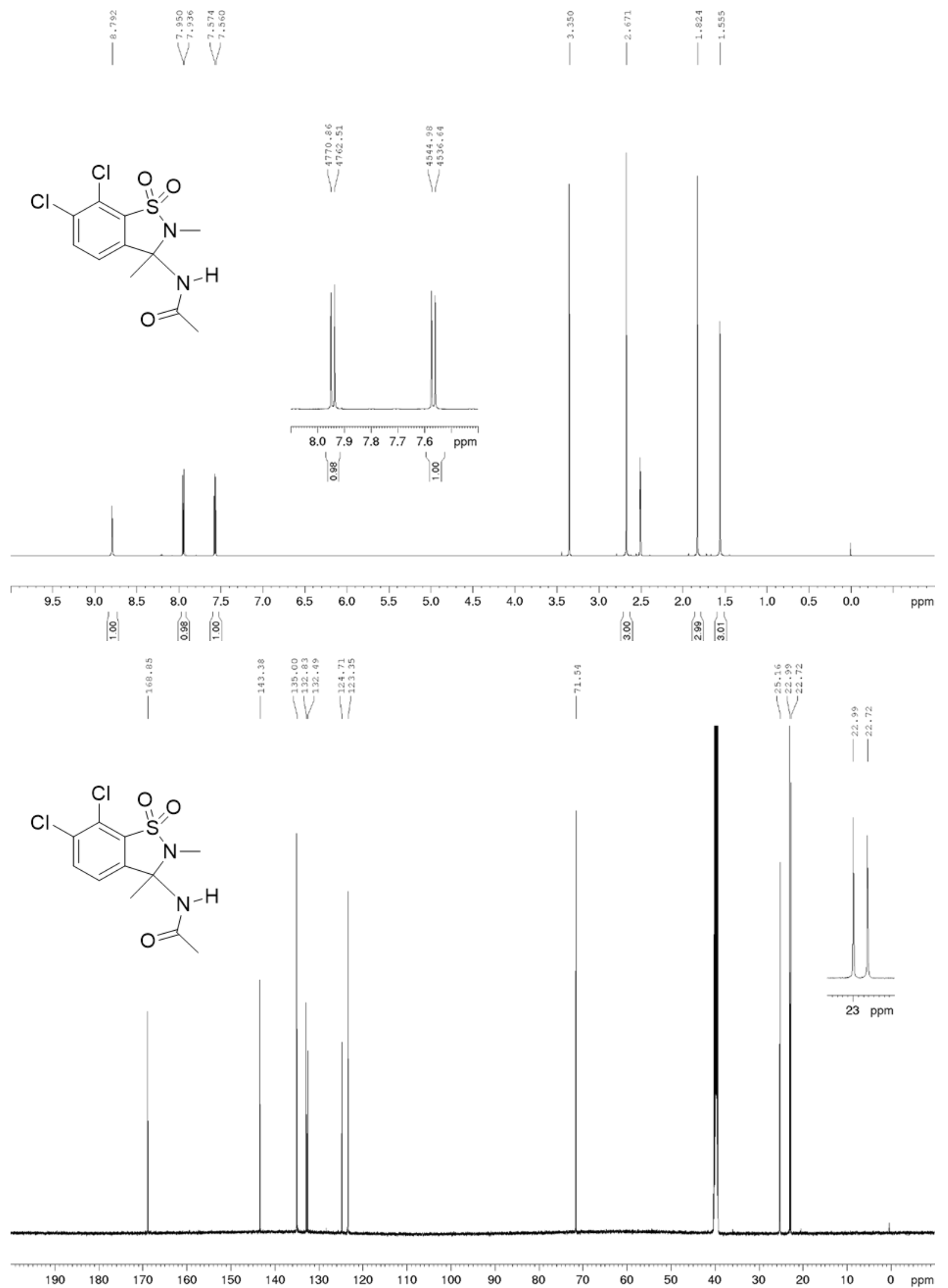
Compound $2 \mathbf{2 b},{ }^{1} \mathrm{H}$ and ${ }^{13} \mathrm{C}$ NMR $\left(600 / 150 \mathrm{MHz},\left[\mathrm{D}_{6}\right] \mathrm{DMSO}\right)$
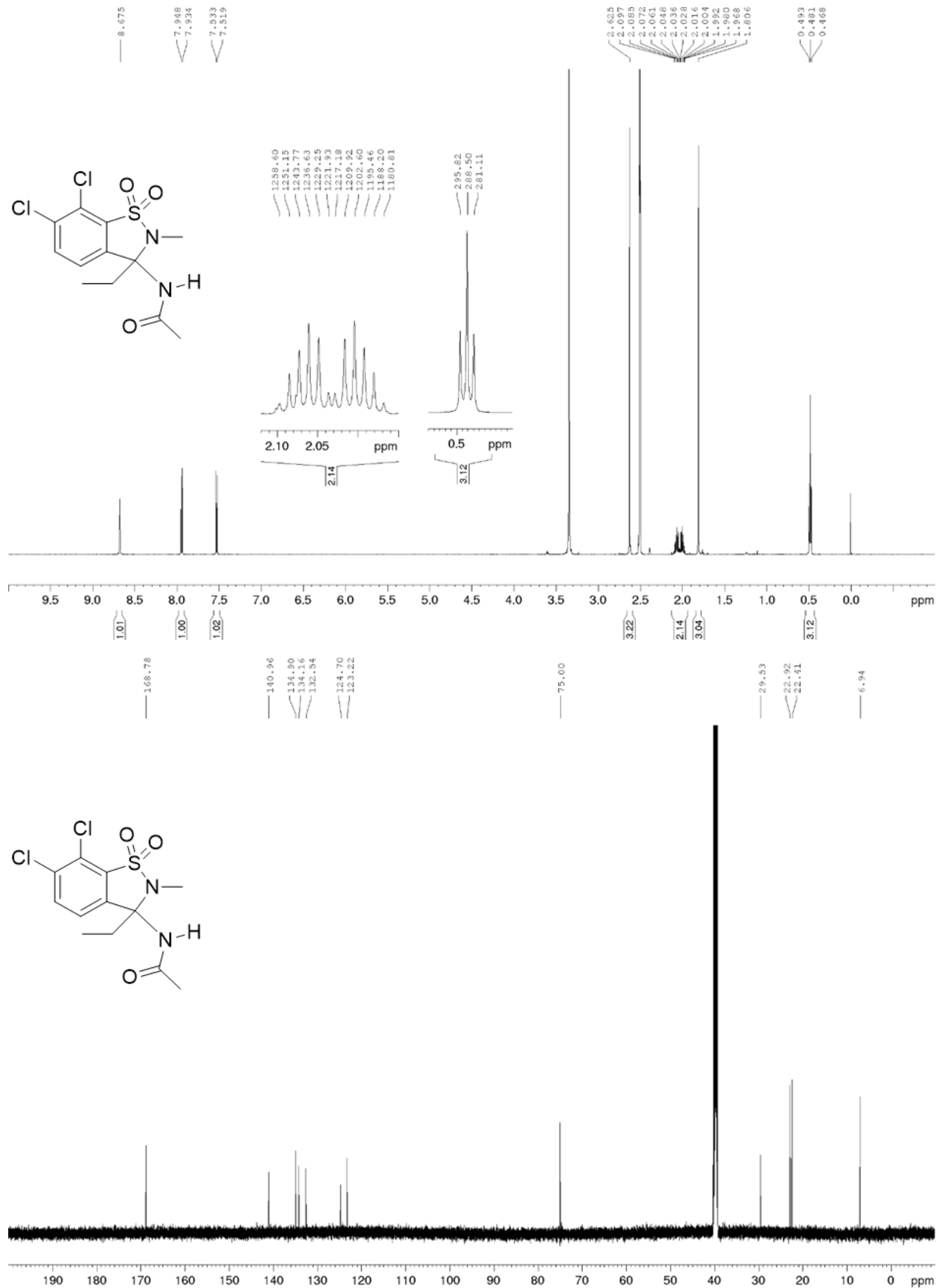
Compound 2c, ${ }^{1} \mathrm{H}$ NMR and DEPTQ $\left(600 / 150 \mathrm{MHz},\left[\mathrm{D}_{6}\right] \mathrm{DMSO}\right)$

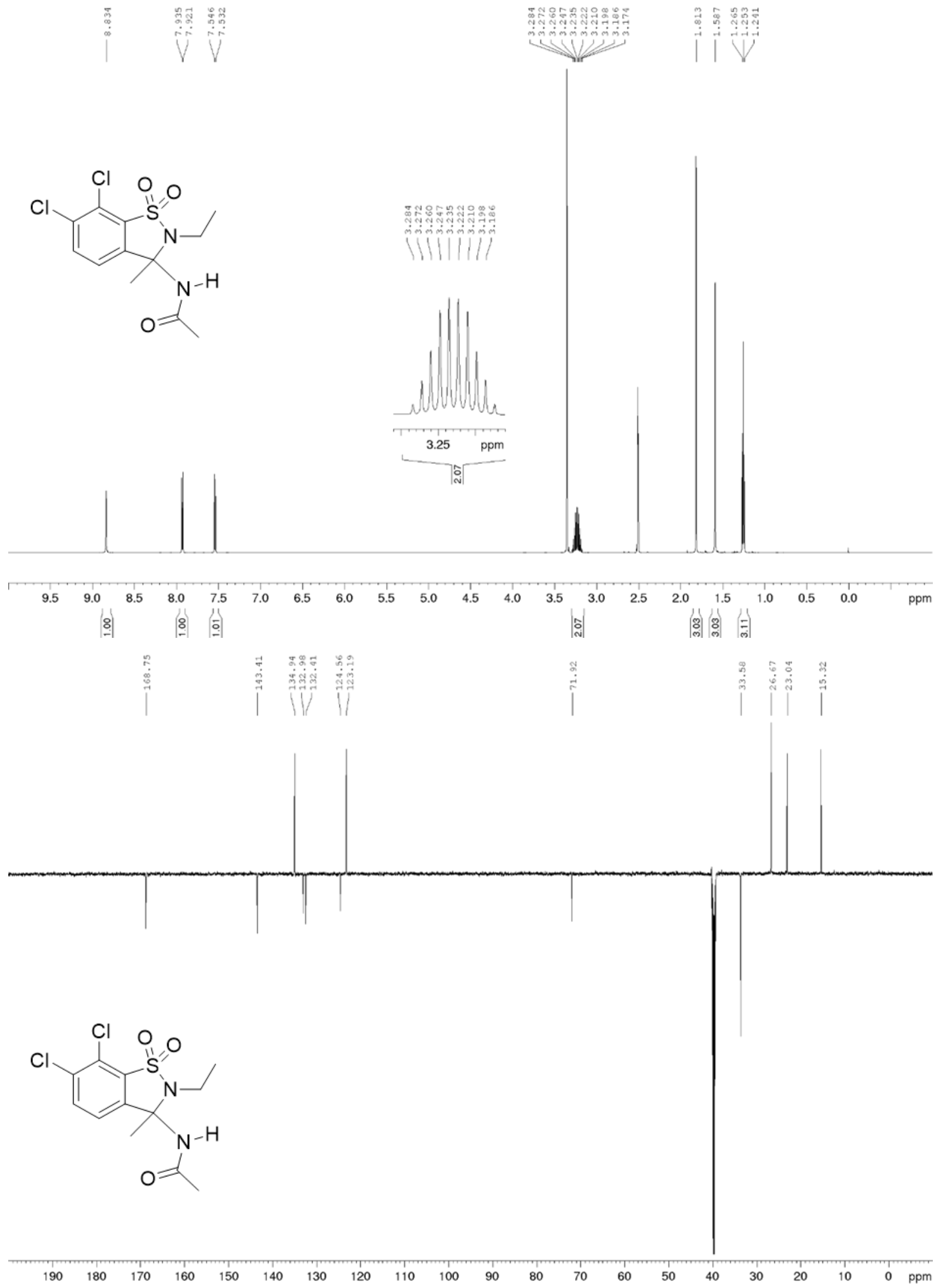


Compound 2d, ${ }^{1} \mathrm{H}$ and ${ }^{13} \mathrm{C}$ NMR $\left(600 / 150 \mathrm{MHz},\left[\mathrm{D}_{6}\right] \mathrm{DMSO}\right)$

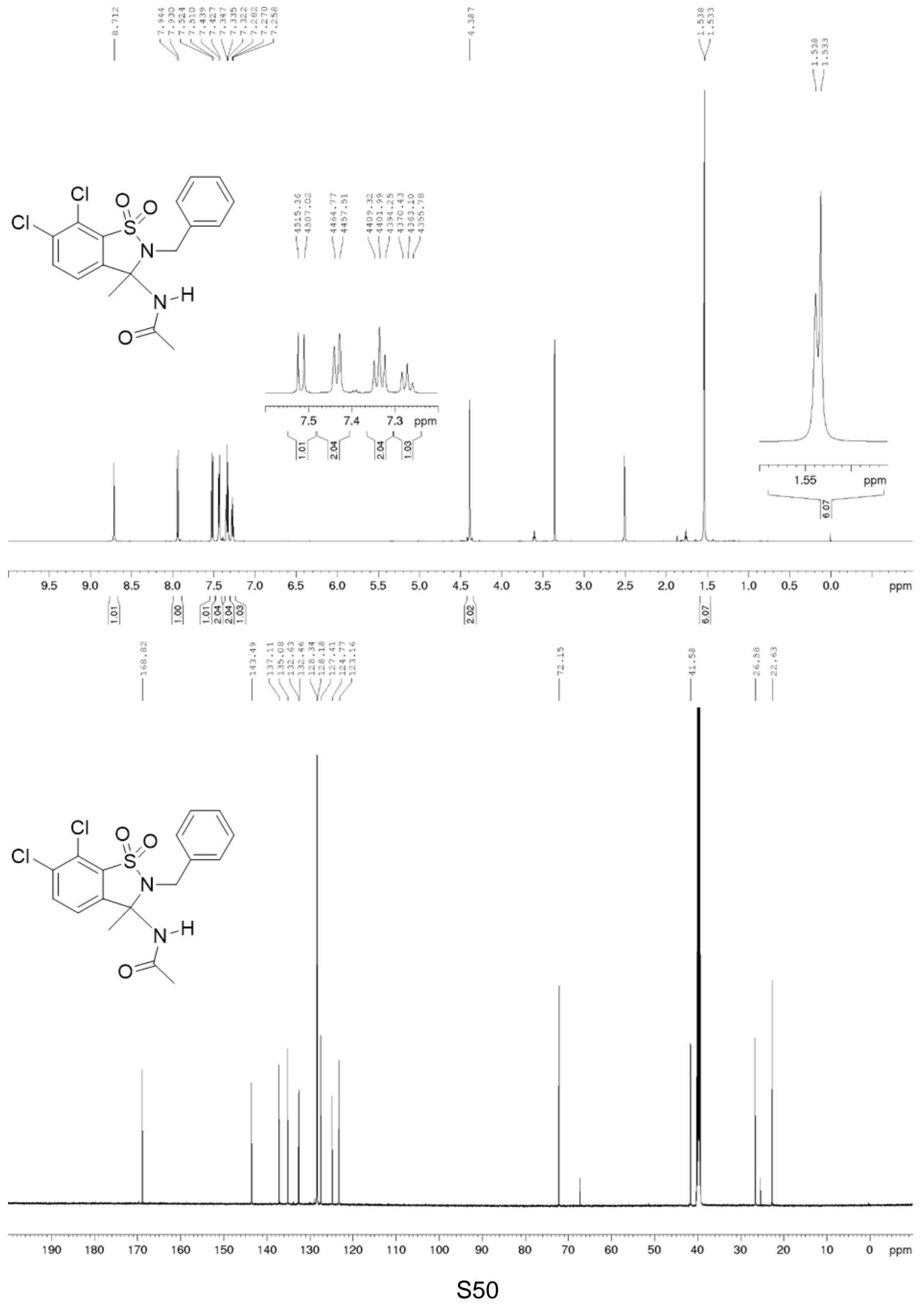


Compound $2 e,{ }^{1} \mathrm{H}$ and ${ }^{13} \mathrm{C}$ NMR $\left(600 / 150 \mathrm{MHz},\left[\mathrm{D}_{6}\right] \mathrm{DMSO}\right)$

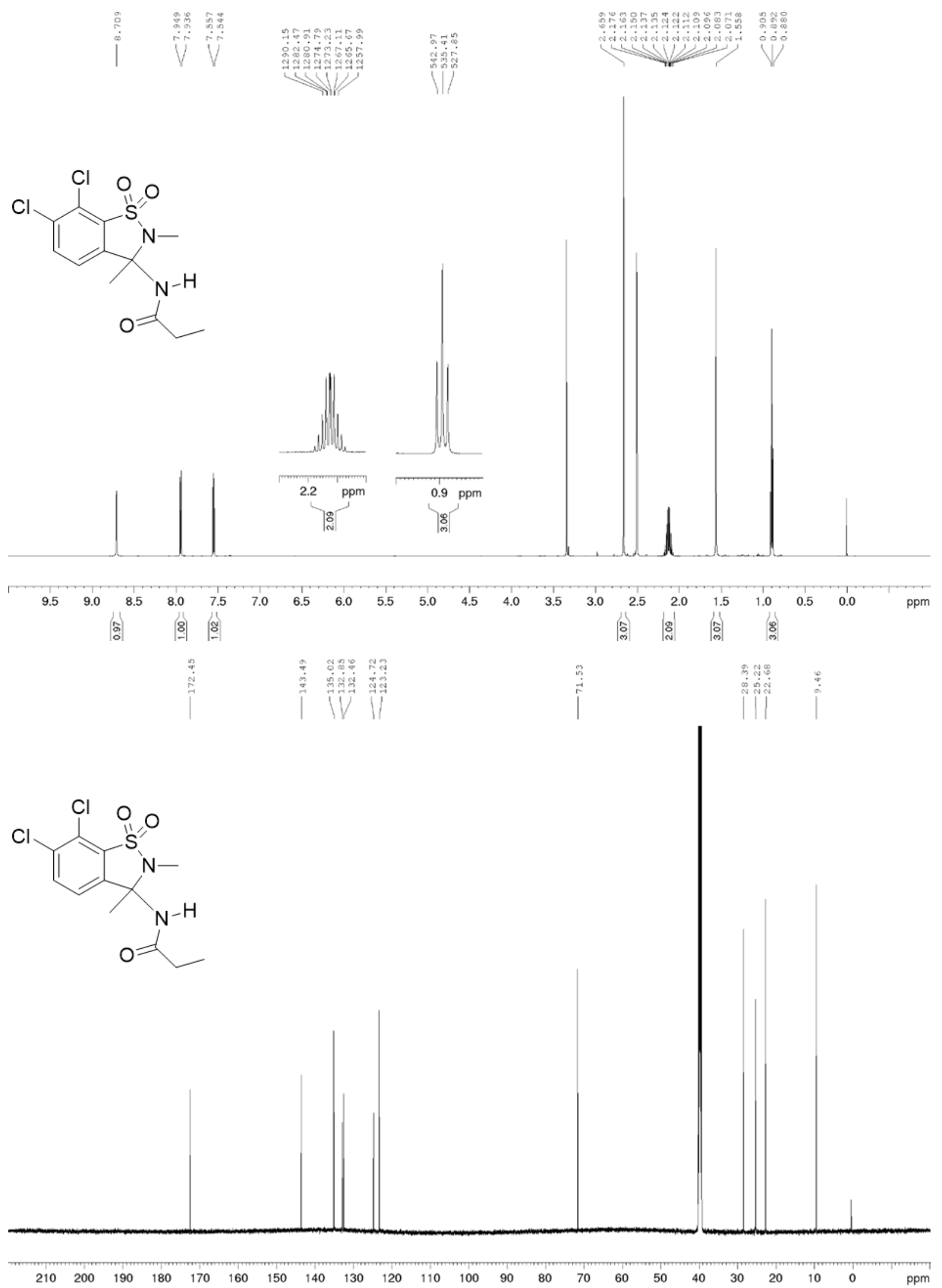


Compound 2f, ${ }^{1} \mathrm{H}$ and ${ }^{13} \mathrm{C}$ NMR $(600 / 150 \mathrm{MHz}$, [D6]DMSO)

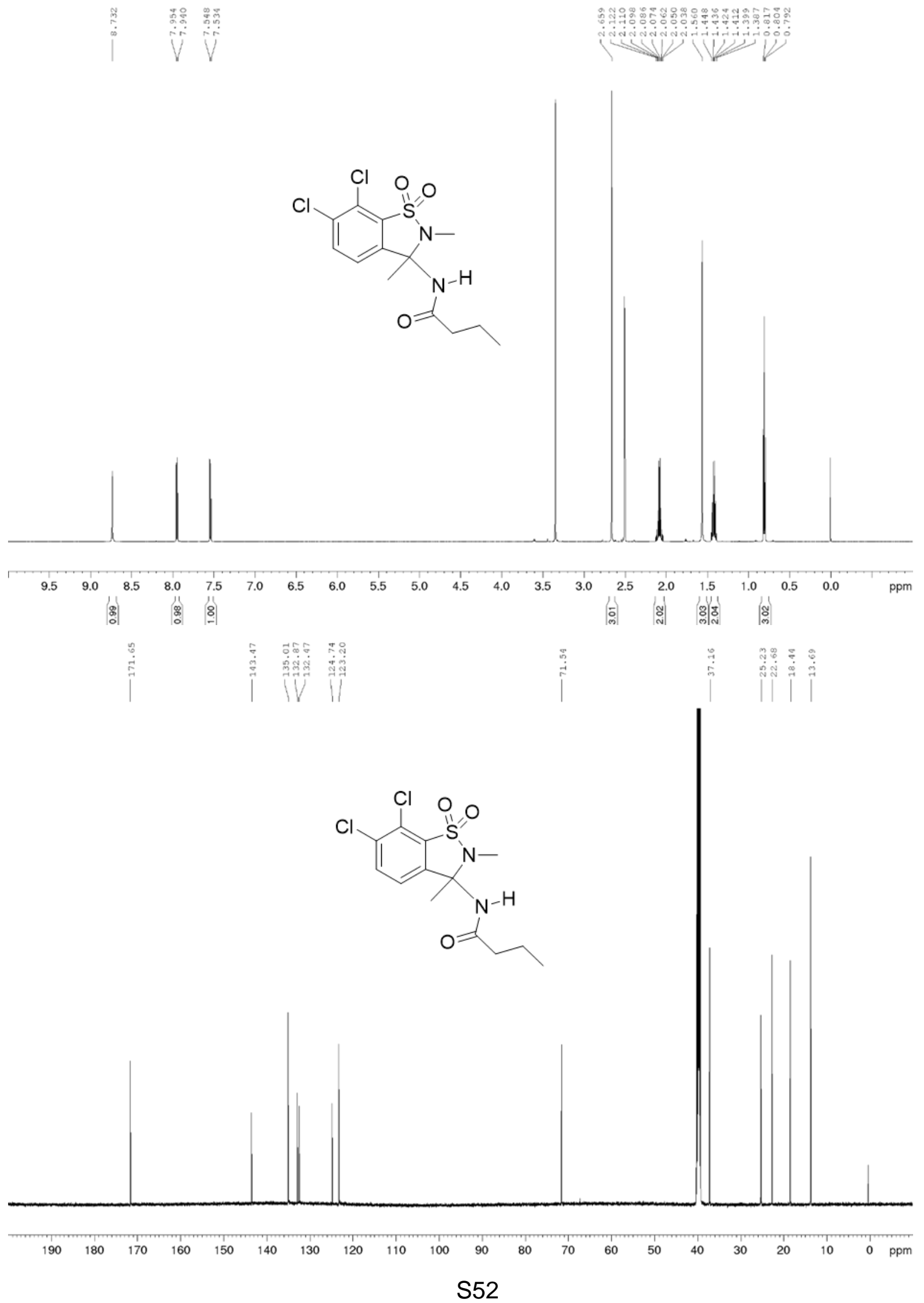


Compound 2g, ${ }^{1} \mathrm{H}$ and ${ }^{13} \mathrm{C}$ NMR $\left(600 / 150 \mathrm{MHz}\left[\mathrm{D}_{6}\right] \mathrm{DMSO}\right)$

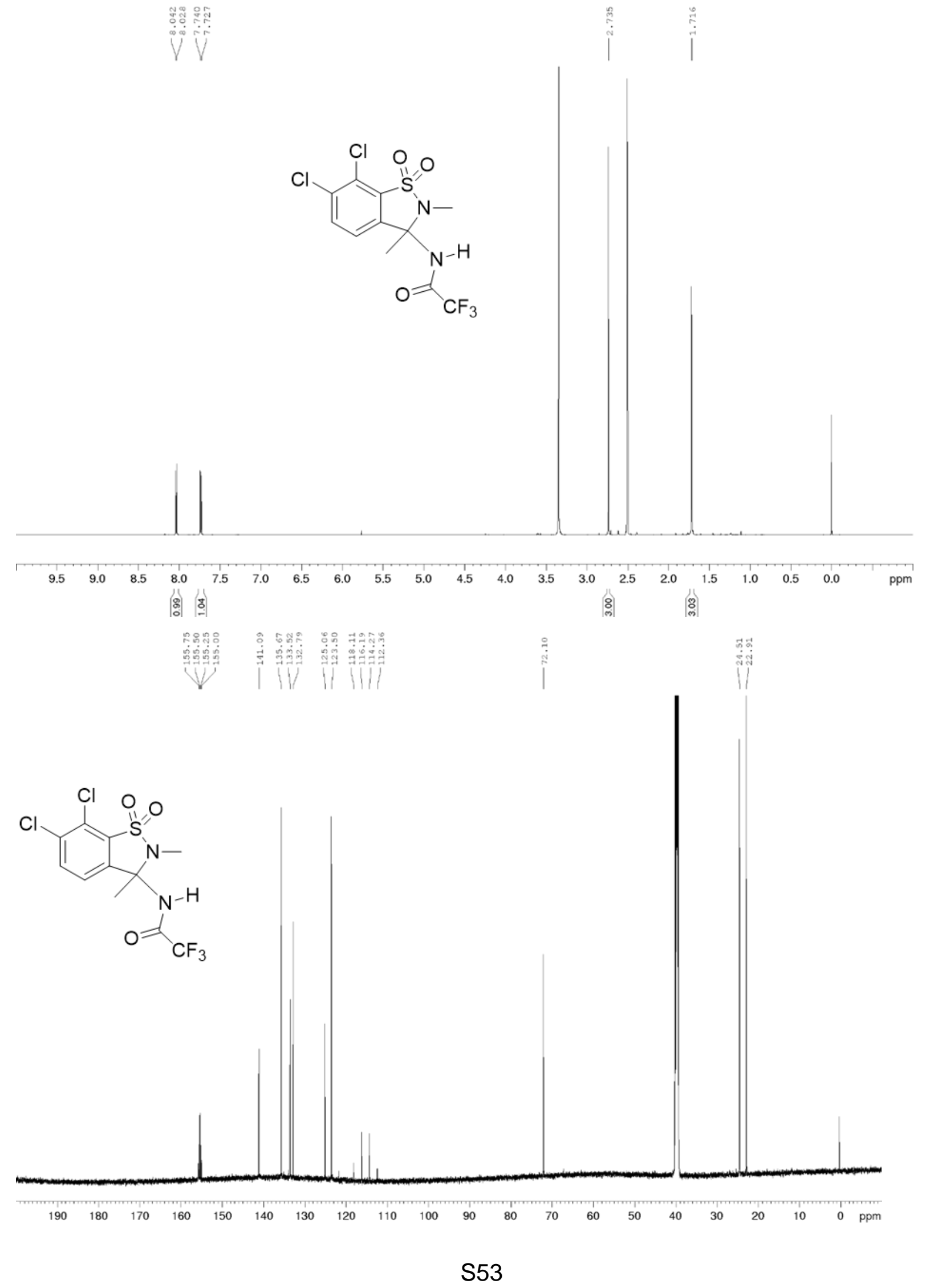


Compound $2 \mathrm{~h},{ }^{1} \mathrm{H}$ and ${ }^{13} \mathrm{C}$ NMR $\left(600 / 150 \mathrm{MHz}\left[\mathrm{D}_{6}\right] \mathrm{DMSO}\right)$

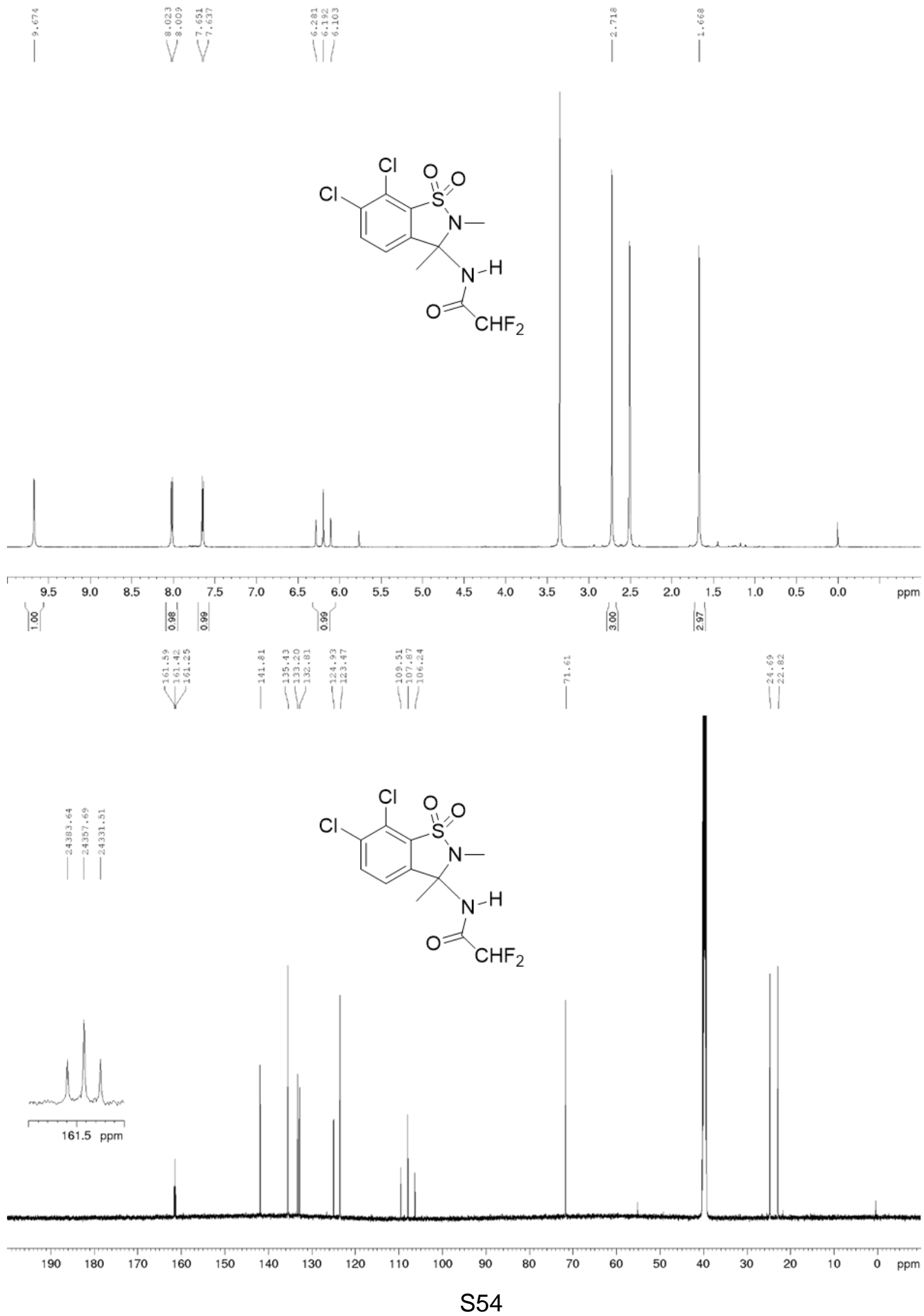


Compound 2i, ${ }^{1} \mathrm{H}$ and ${ }^{13} \mathrm{C}$ NMR $\left(600 / 150 \mathrm{MHz},\left[\mathrm{D}_{6}\right] \mathrm{DMSO}\right)$

V
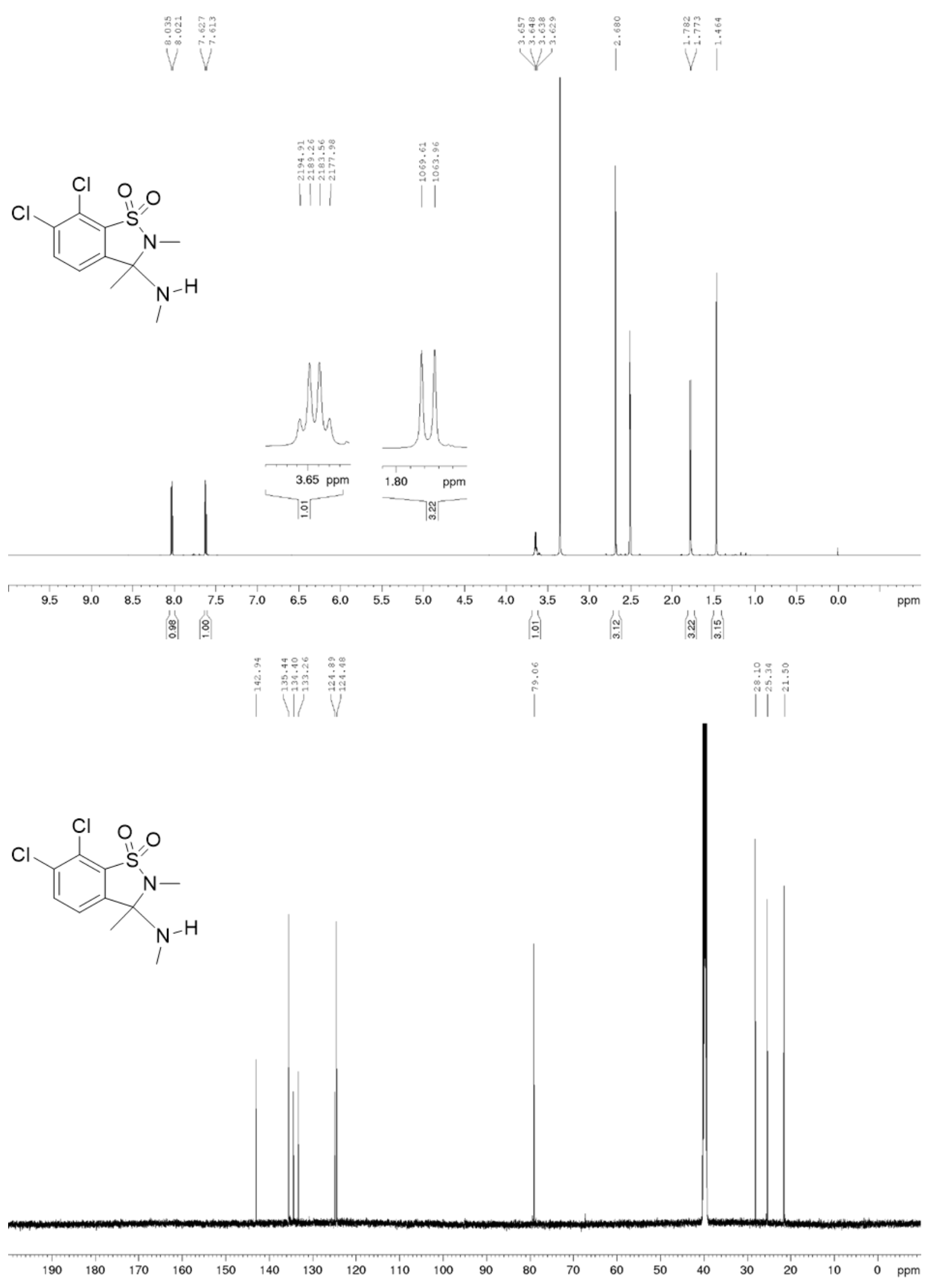
Compound 2j, ${ }^{1} \mathrm{H}$ and ${ }^{13} \mathrm{C}$ NMR $(600 / 150 \mathrm{MHz}$, [D6]DMSO)

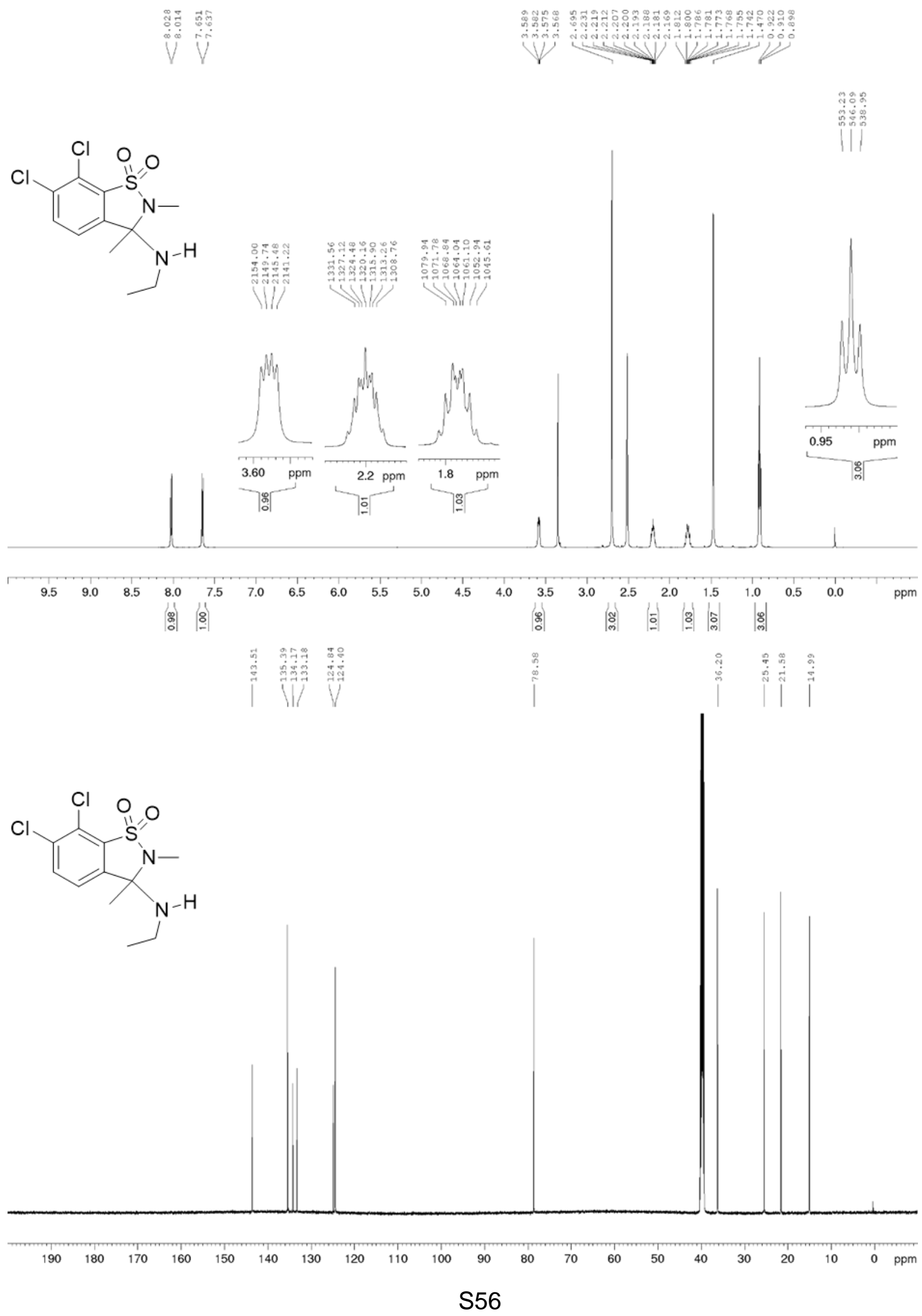


Compound 2k, ${ }^{1} \mathrm{H}$ and ${ }^{13} \mathrm{C}$ NMR $\left(600 / 150 \mathrm{MHz},\left[\mathrm{D}_{6}\right] \mathrm{DMSO}\right)$

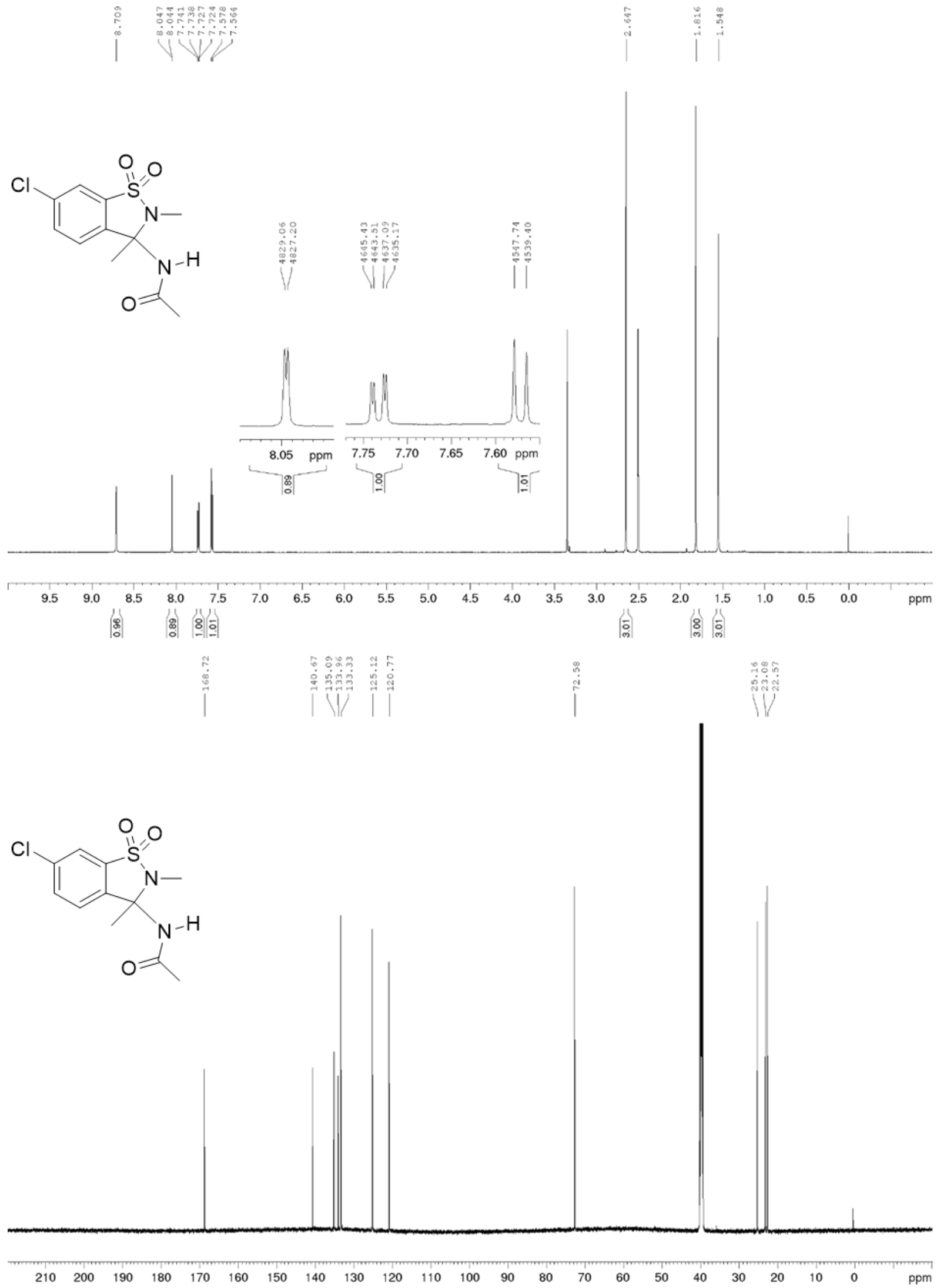


Compound 2I, ${ }^{1} \mathrm{H}$ and ${ }^{13} \mathrm{C}$ NMR $(600 / 150 \mathrm{MHz}$, [D6]DMSO)

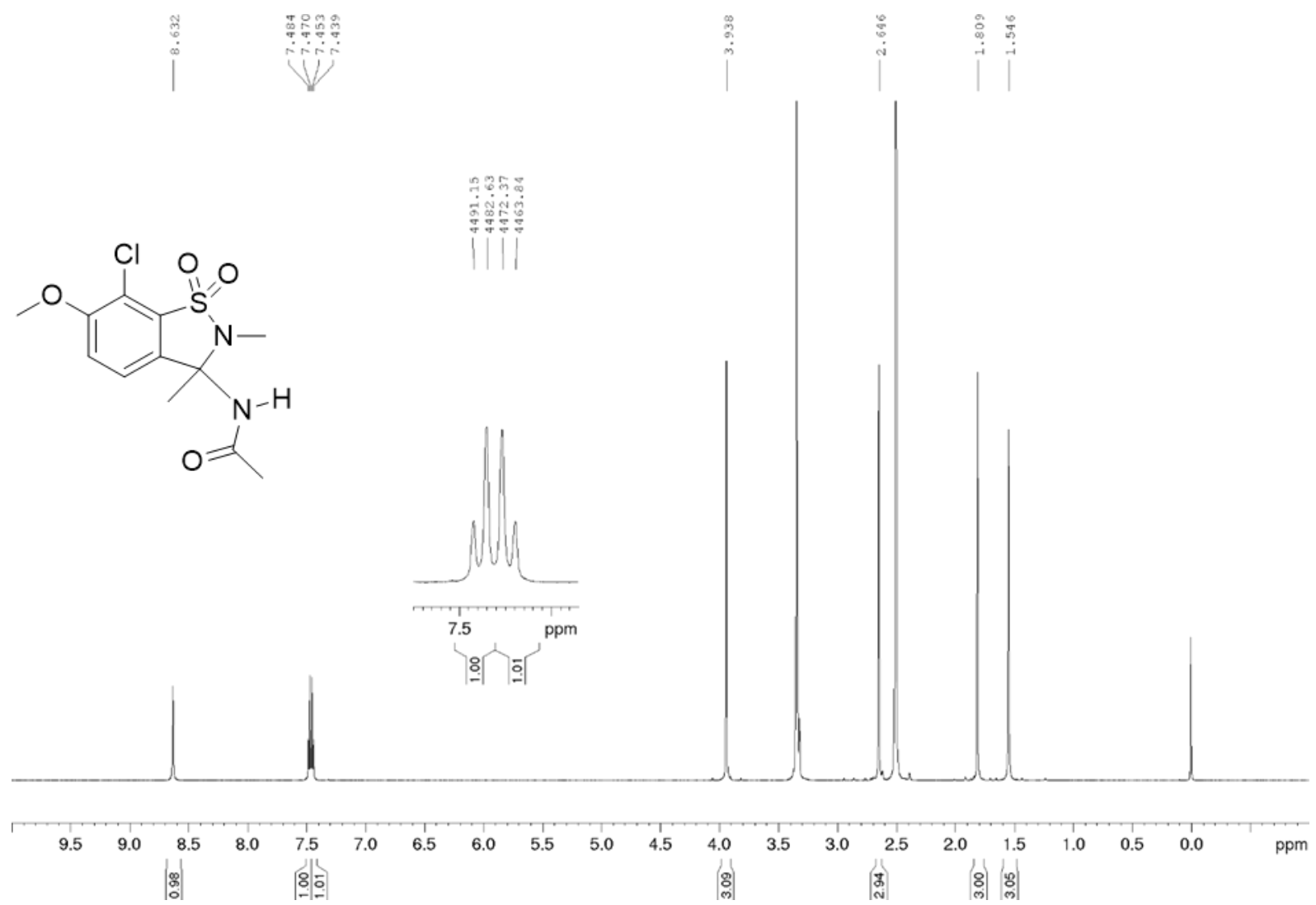
|<smiles>COc1ccc2c(c1Cl)S(=O)(=O)N(C)C2(C)NC(C)=O</smiles>

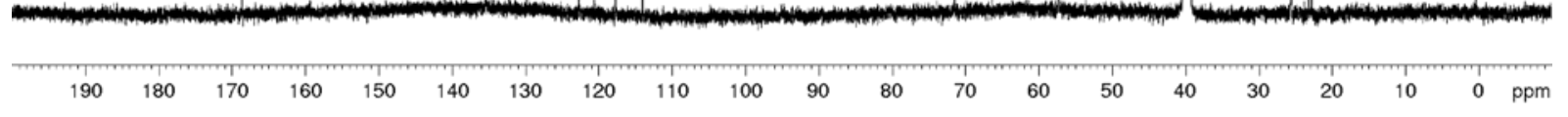
S58 
Compound $2 \mathrm{~m},{ }^{1} \mathrm{H}$ and ${ }^{13} \mathrm{C}$ NMR $\left(600 / 150 \mathrm{MHz},\left[\mathrm{D}_{6}\right] \mathrm{DMSO}\right)$
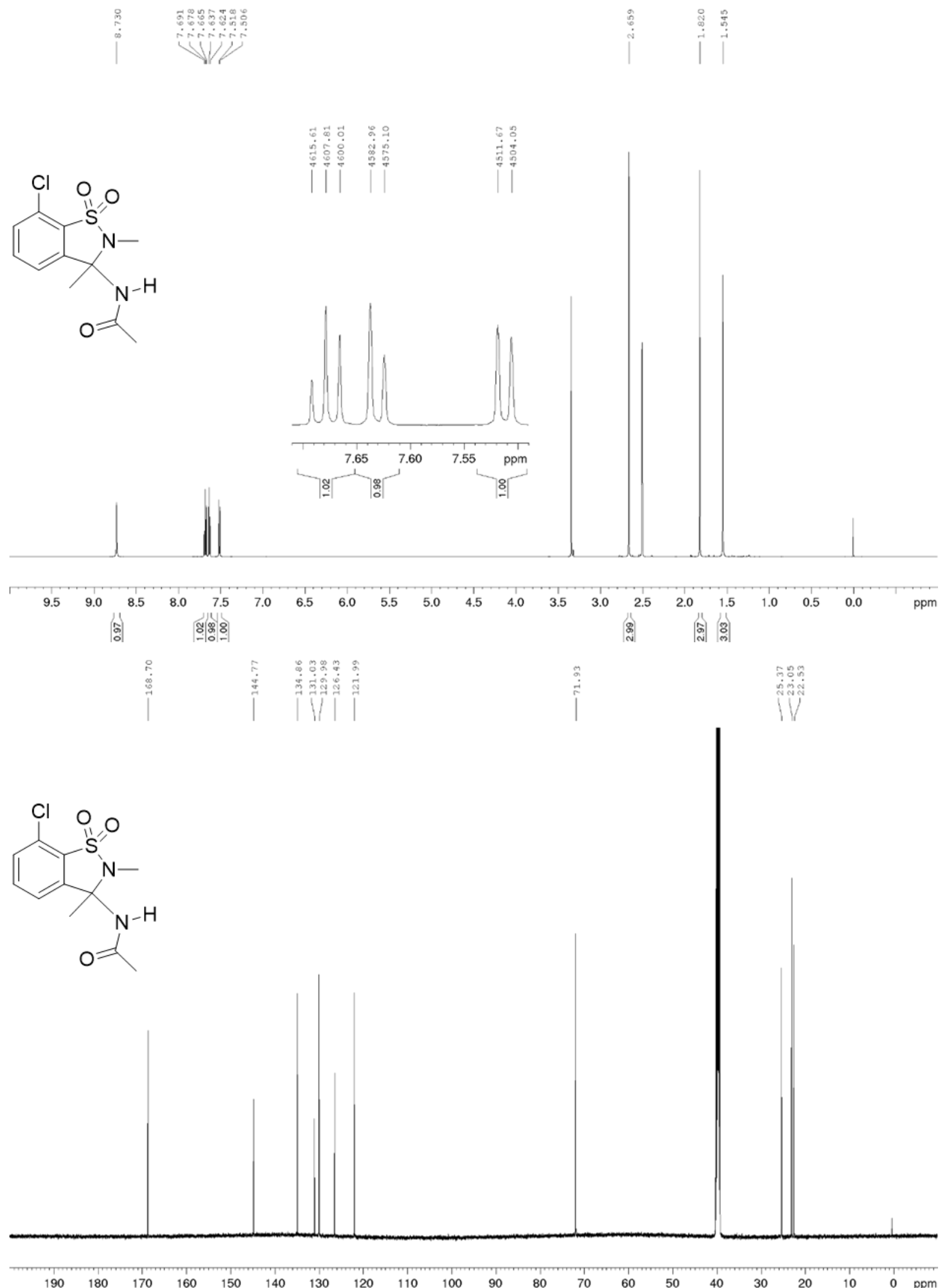
S59 
Compound 2n, ${ }^{1} \mathrm{H}$ and ${ }^{13} \mathrm{C}$ NMR $\left(600 / 150 \mathrm{MHz},\left[\mathrm{D}_{6}\right] \mathrm{DMSO}\right)$

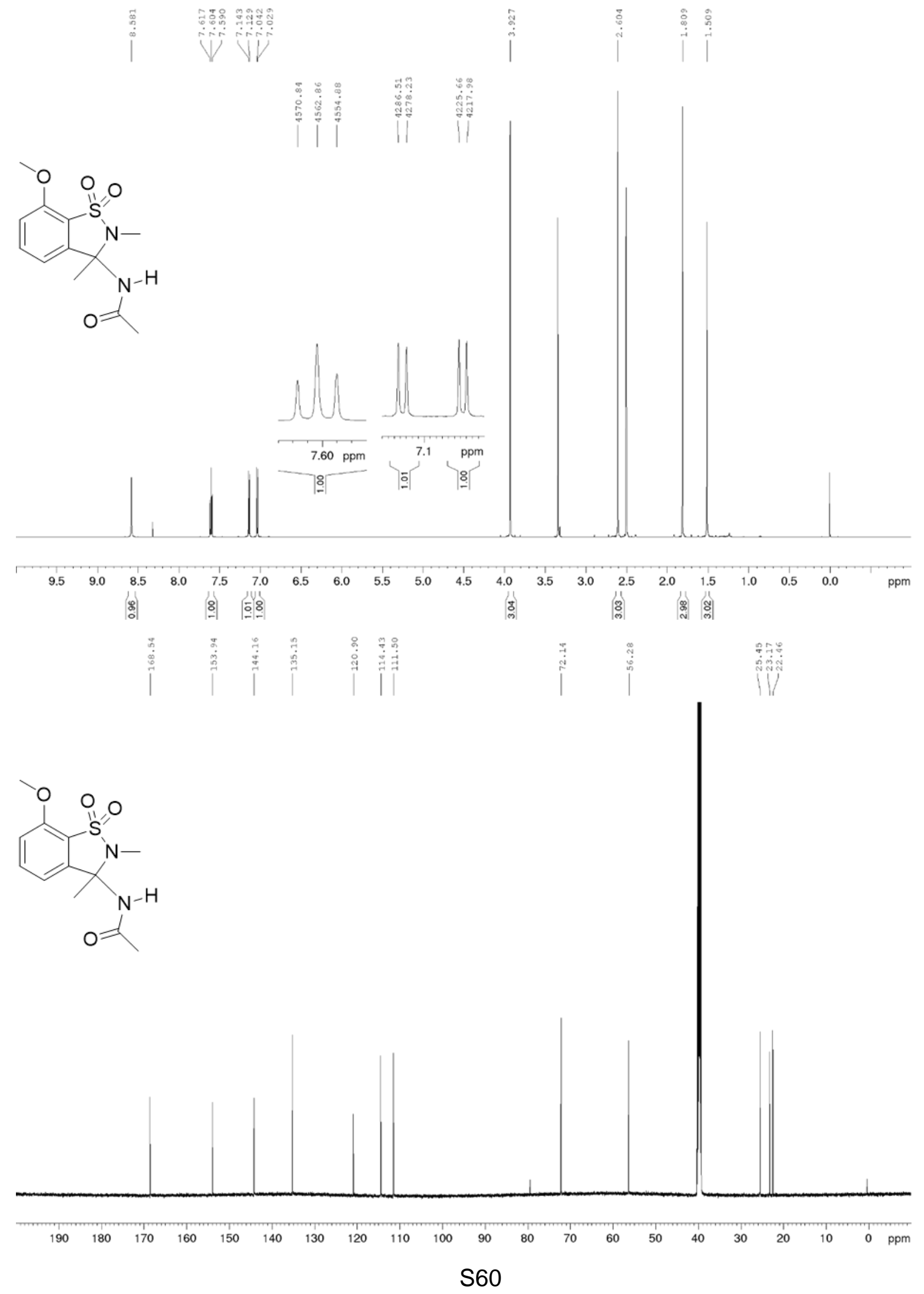


Compound 2o, ${ }^{1} \mathrm{H}$ and ${ }^{13} \mathrm{C}$ NMR $\left(600 / 150 \mathrm{MHz},\left[\mathrm{D}_{6}\right] \mathrm{DMSO}\right)$

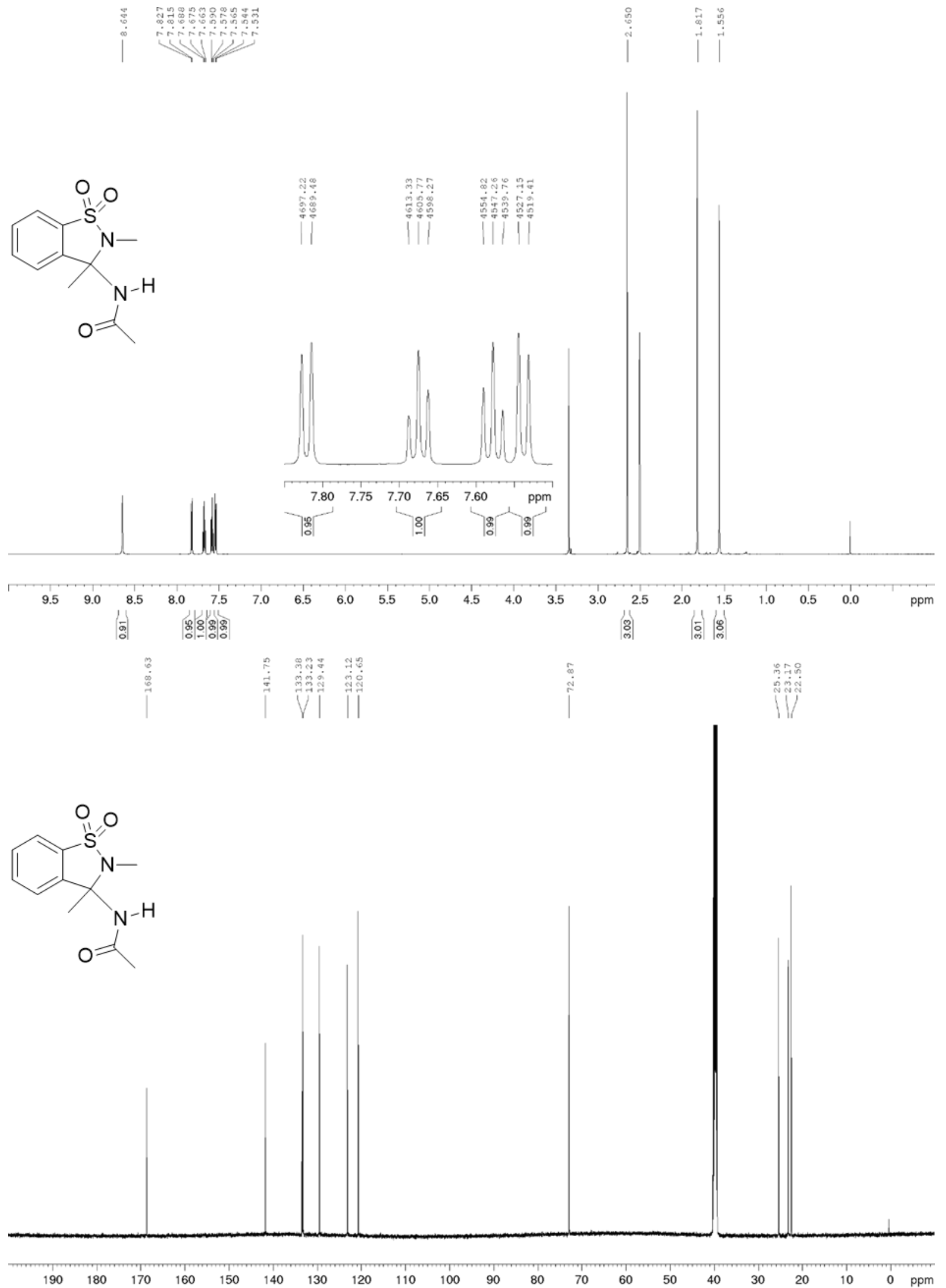


Compound iq, ${ }^{1} \mathrm{H}$ and ${ }^{13} \mathrm{C}$ NMR $(600 / 150 \mathrm{MHz}$, [De ]DMSO)
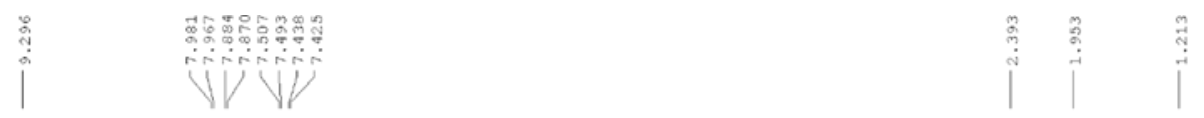<smiles>CC(=O)NC1(C)c2ccc(Cl)c(Cl)c2S(=O)(=O)N1S(=O)(=O)c1ccc(C)cc1</smiles>
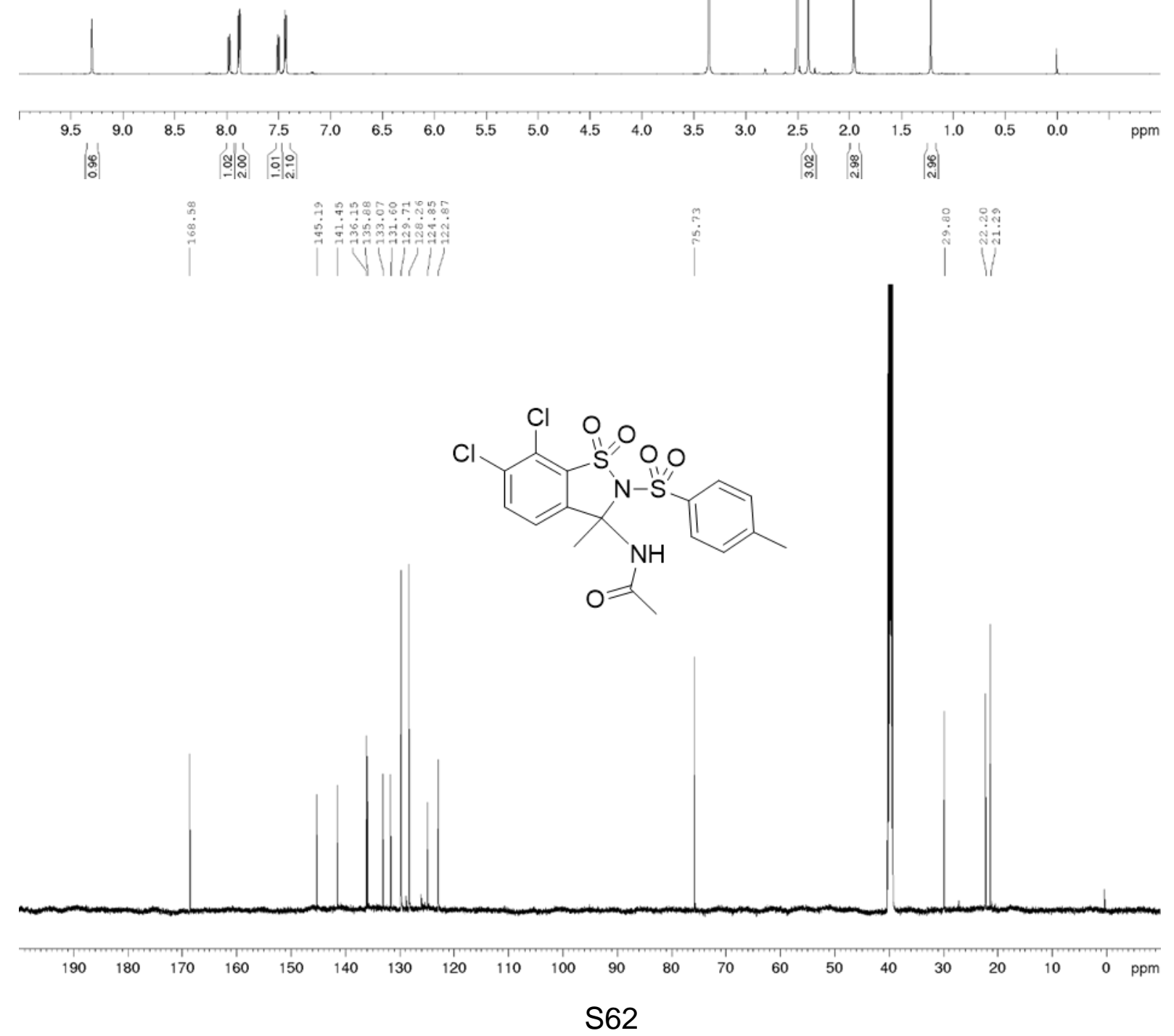
Compound 5q, ${ }^{1} \mathrm{H}$ and ${ }^{13} \mathrm{C}$ NMR $\left(600 / 150 \mathrm{MHz},\left[\mathrm{D}_{6}\right] \mathrm{DMSO}\right)$

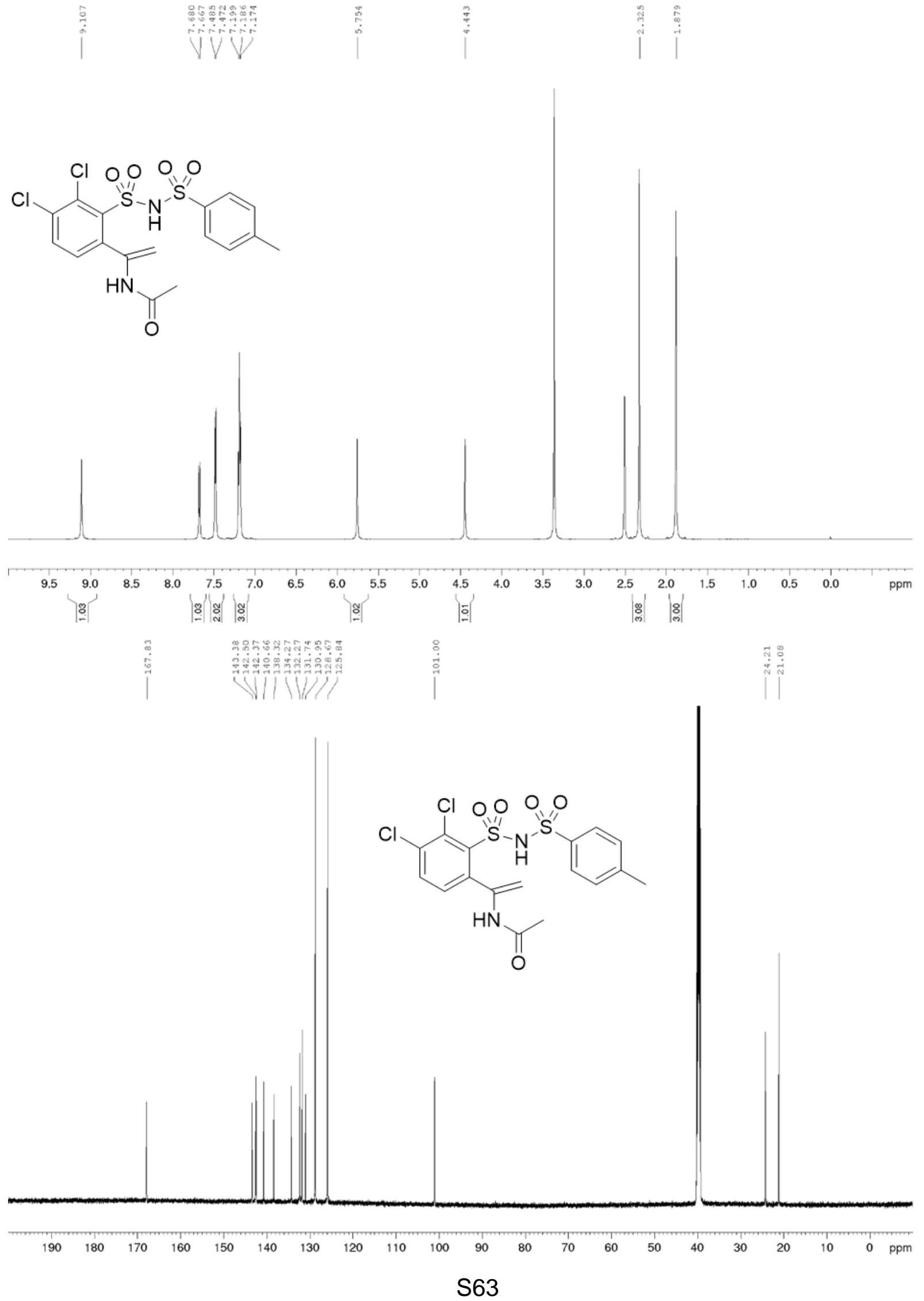


Compound 3a, ${ }^{1} \mathrm{H}$ and ${ }^{13} \mathrm{C}$ NMR $\left(600 / 150 \mathrm{MHz},\left[\mathrm{D}_{6}\right] \mathrm{DMSO}\right)$

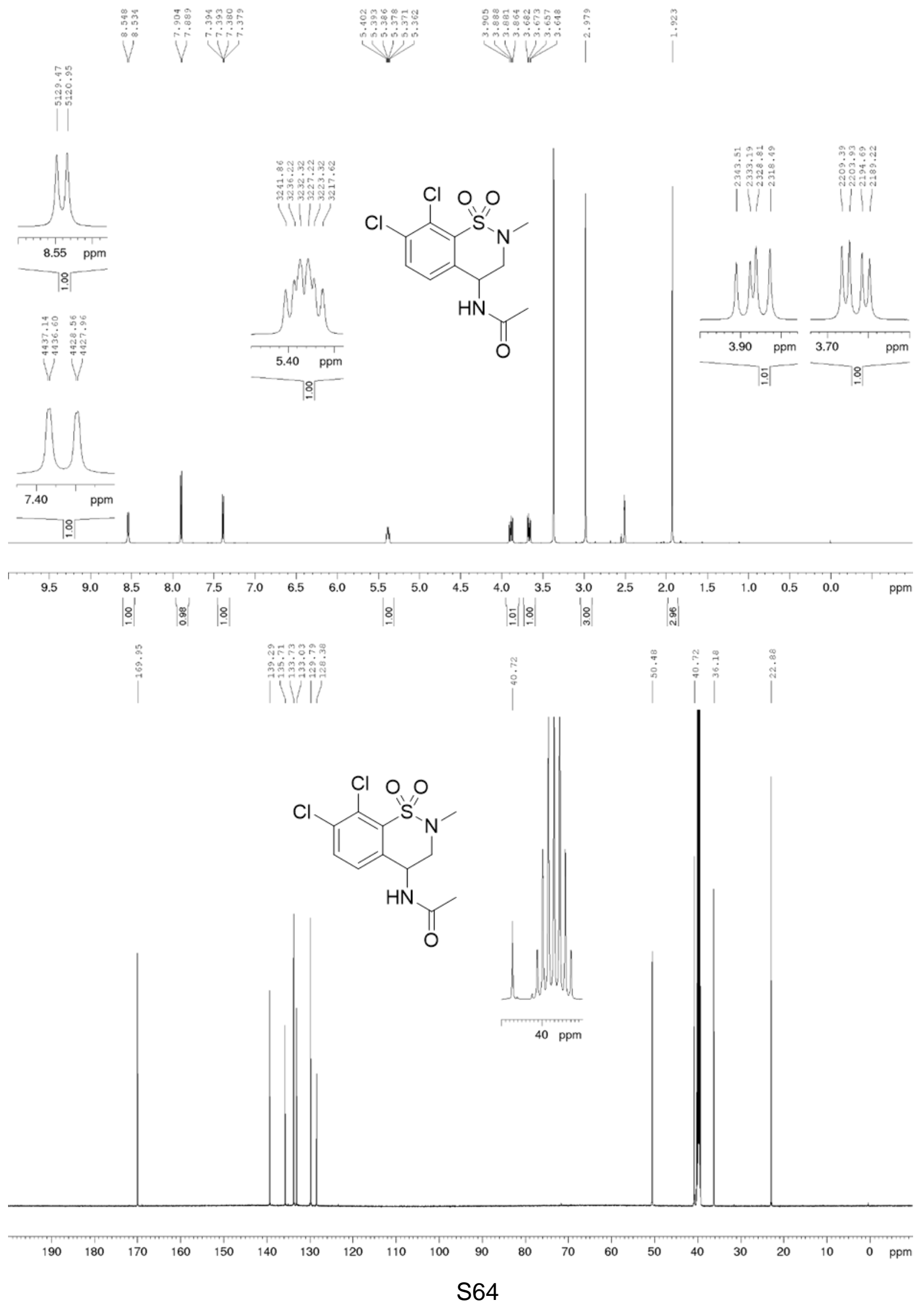


Compound trans-3b, ${ }^{1} \mathrm{H}$ NMR and DEPTQ $\left(600 / 150 \mathrm{MHz},\left[\mathrm{D}_{6}\right] \mathrm{DMSO}\right)$

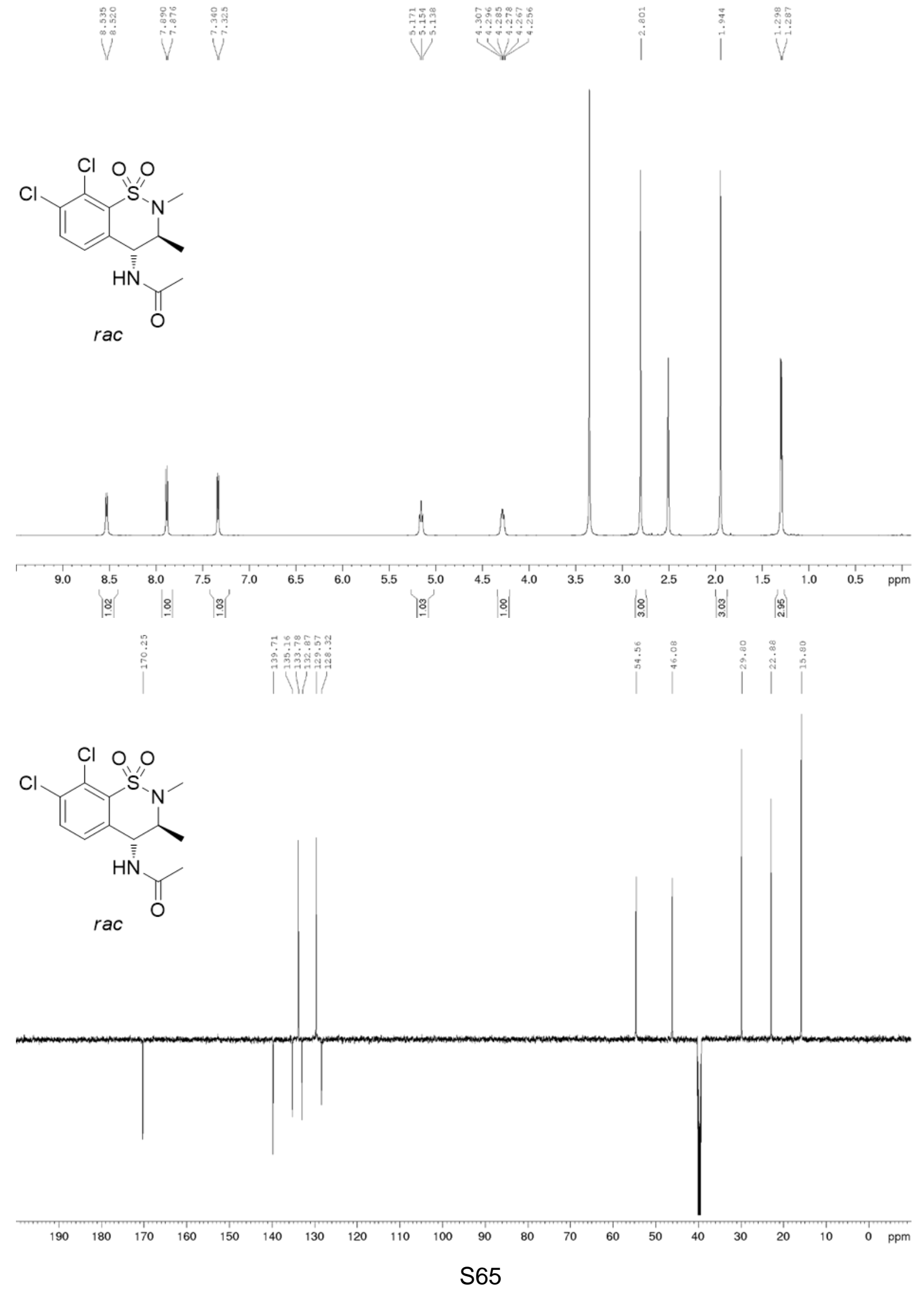


Compound 3c, ${ }^{1} \mathrm{H}$ and ${ }^{13} \mathrm{C}$ NMR $\left(600 / 150 \mathrm{MHz},\left[\mathrm{D}_{6}\right] \mathrm{DMSO}\right)$

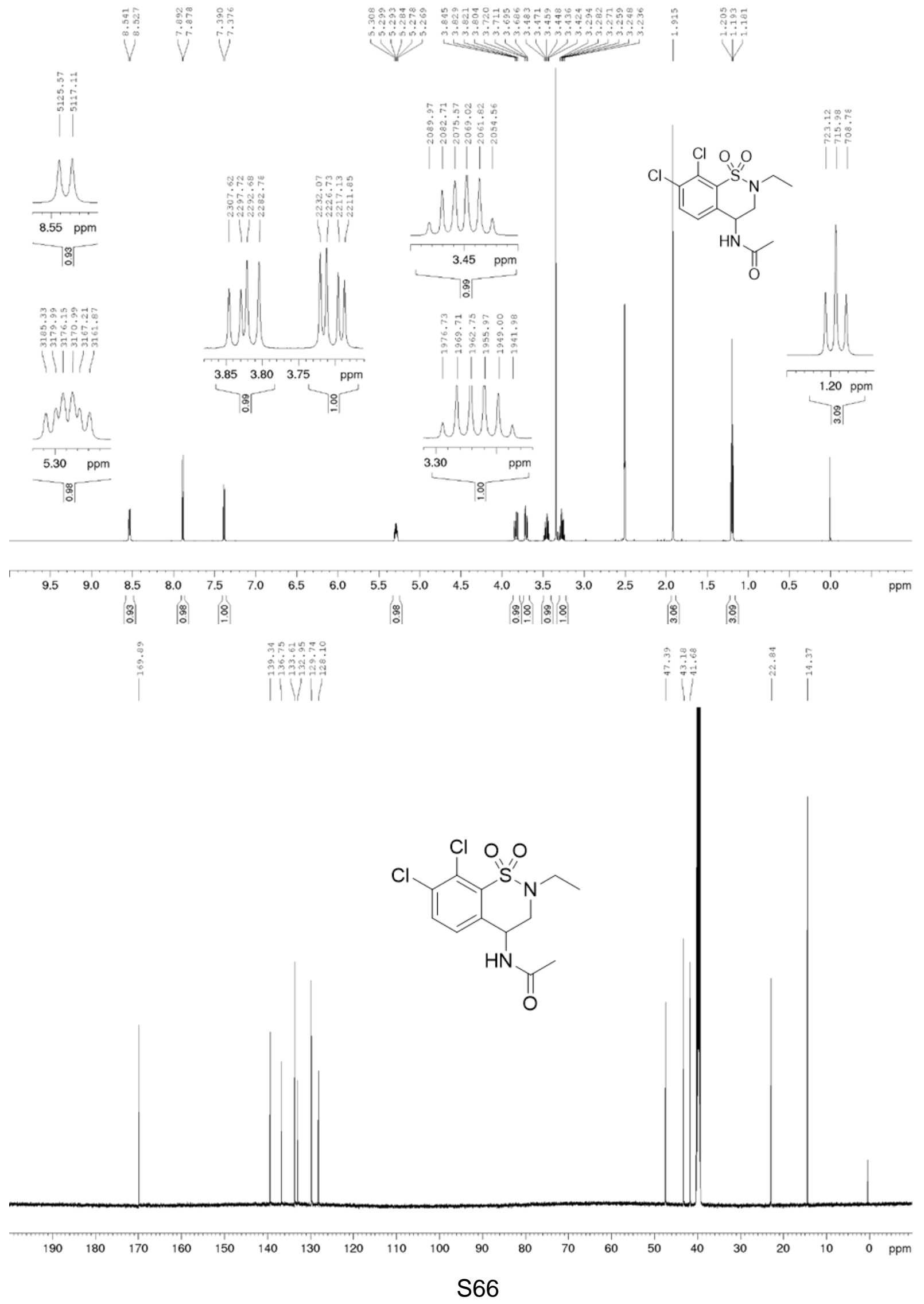


Compound 3d, ${ }^{1} \mathrm{H}$ and ${ }^{13} \mathrm{C}$ NMR $\left(600 / 150 \mathrm{MHz},\left[\mathrm{D}_{6}\right.\right.$ ]DMSO)

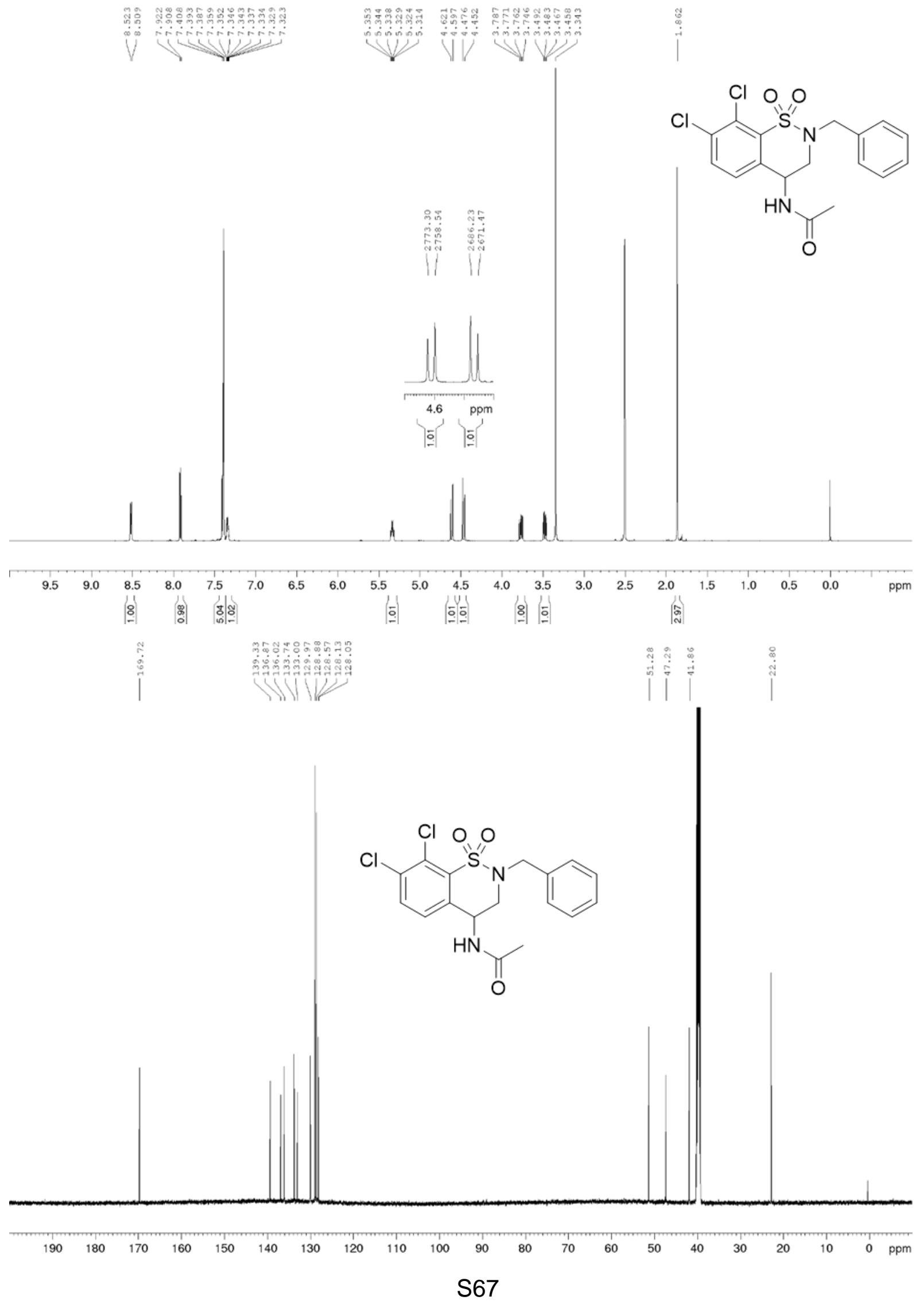


Compound 3e, ${ }^{1} \mathrm{H}$ and ${ }^{13} \mathrm{C}$ NMR $\left(600 / 150 \mathrm{MHz},\left[\mathrm{D}_{6}\right] \mathrm{DMSO}\right)$

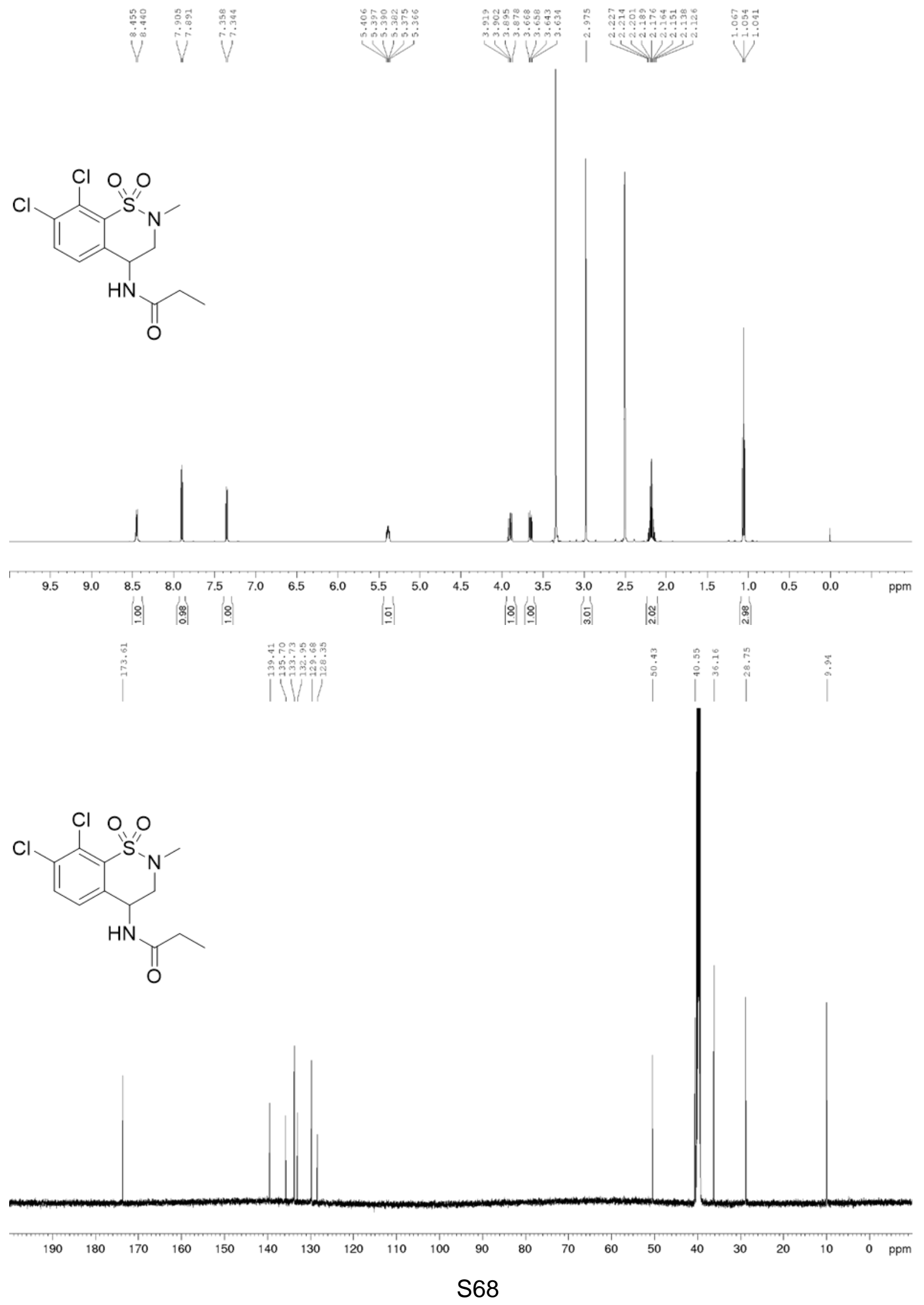


Compound 3f, ${ }^{1} \mathrm{H}$ and ${ }^{13} \mathrm{C}$ NMR $(600 / 150 \mathrm{MHz}$, [D6]DMSO)

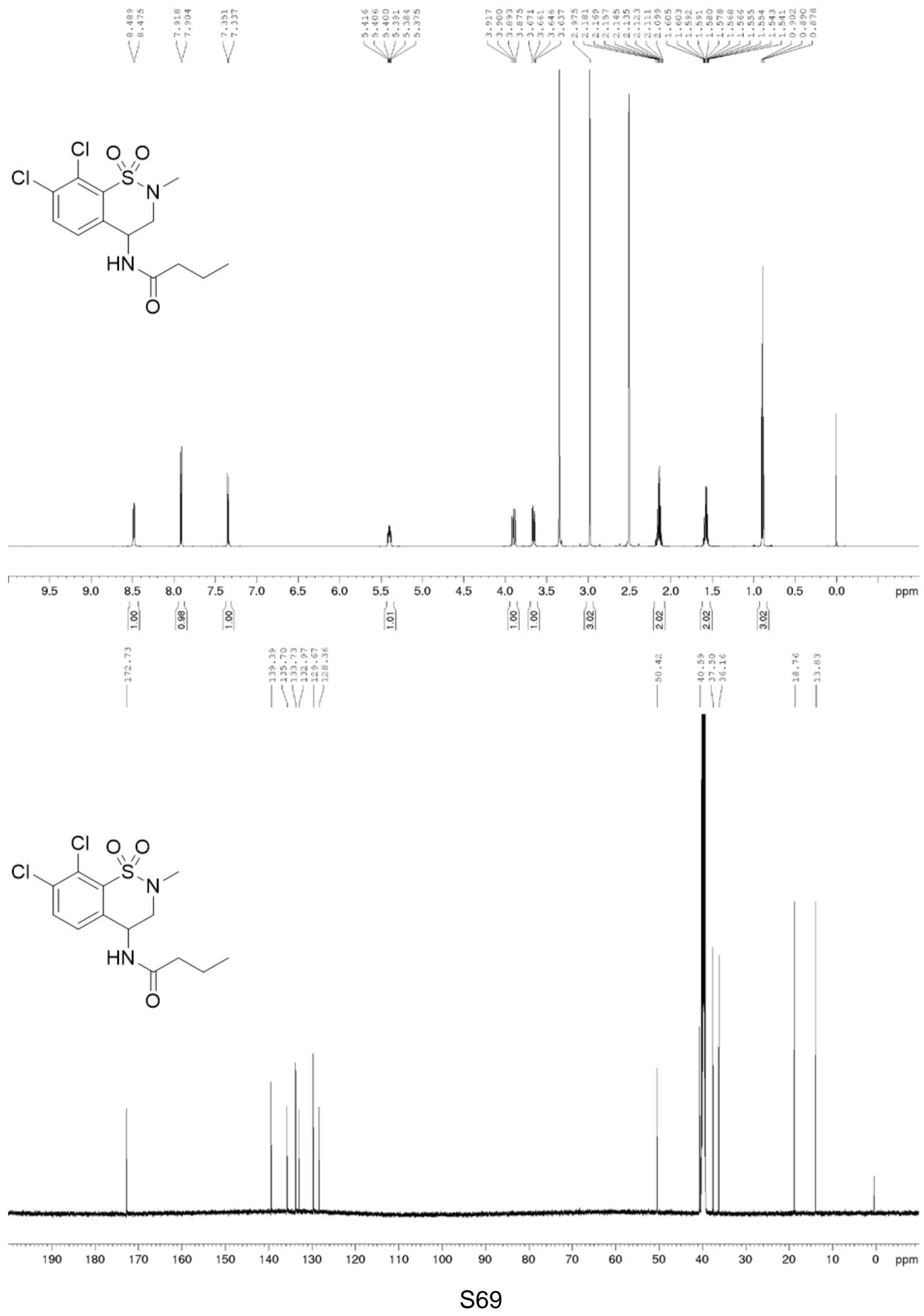




\section{Calculated total energies and $X Y Z$ coordinates}

M06-2X/6-31+G* (smd: THF) with ultrafine grid

\section{$1 a$}<smiles>CC(=O)N1C(C)c2ccc(Cl)c(Cl)c2S(=O)(=O)N1C</smiles>

$$
E=-2079.7081933
$$

$\begin{array}{llll}\text { C } & 0.000090 & -0.019833 & 1.529249\end{array}$

$\begin{array}{llll}\text { C } & 1.144447 & -0.012415 & 2.339050\end{array}$

$\begin{array}{llll}\mathrm{S} & 2.760229 & 0.138562 & 1.565958\end{array}$

$\begin{array}{llll}N & 2.341380 & 0.757175 & 0.064308\end{array}$

$\begin{array}{llll}\text { N } & 1.373019 & -0.037576 & -0.525034\end{array}$

$\begin{array}{llll}\text { C } & 1.053364 & -0.119378 & 3.735920\end{array}$

$\begin{array}{llll}\text { C } & -0.204563 & -0.173199 & 4.332850\end{array}$

$\begin{array}{llll}\text { C } & -1.351910 & -0.146627 & 3.543059\end{array}$

$\begin{array}{llll}\text { C } & -1.245820 & -0.081377 & 2.164509\end{array}$

$\begin{array}{llll}\text { Cl } & 2.475627 & -0.230919 & 4.714895\end{array}$

$\begin{array}{llll}\text { Cl } & -0.376916 & -0.290254 & 6.054228\end{array}$

\begin{tabular}{llll}
\hline & 3.545670 & 1.175093 & 2.215277
\end{tabular}

$\begin{array}{llll}0 & 3.307340 & -1.194286 & 1.374170\end{array}$

$\begin{array}{llll}\text { C } & 2.164486 & 2.217339 & -0.030406\end{array}$

$\begin{array}{llll}\text { C } & 1.689230 & -0.742363 & -1.671617\end{array}$

$\begin{array}{llll}\text { C } & 3.113877 & -0.643859 & -2.149750\end{array}$

$\begin{array}{llll}\text { C } & -0.858878 & 1.057835 & -0.620733\end{array}$

$\begin{array}{llll}0 & 0.827974 & -1.396900 & -2.239044\end{array}$

$\begin{array}{llll}\mathrm{H} & -2.326658 & -0.198640 & 4.017512\end{array}$

$\begin{array}{llll}H & -2.152398 & -0.103717 & 1.567928\end{array}$

$\begin{array}{llll}H & -0.401898 & -1.009235 & -0.291574\end{array}$

$\begin{array}{llll}H & 1.400912 & 2.598495 & 0.656969\end{array}$

$\begin{array}{llll}H & 3.120329 & 2.694976 & 0.181152\end{array}$

$\begin{array}{llll}H & 1.873740 & 2.427217 & -1.061746\end{array}$

$\begin{array}{llll}H & 3.382685 & 0.395592 & -2.361005\end{array}$

$\begin{array}{llll}H & 3.803511 & -1.016870 & -1.387565\end{array}$

$\begin{array}{llll}H & 3.205115 & -1.240368 & -3.057727\end{array}$

$\begin{array}{llll}H & -1.915949 & 0.883888 & -0.404261\end{array}$

$\begin{array}{llll}H & -0.592328 & 2.051390 & -0.252527\end{array}$

$\begin{array}{llll}H & -0.731349 & 1.032043 & -1.706952\end{array}$ 
$2 a$<smiles>CC(=O)NC1(C)c2ccc(Cl)c(Cl)c2S(=O)(=O)N1C</smiles>

\section{$E=-2079.7458281$}

\begin{tabular}{|c|c|c|c|}
\hline C & -0.007597 & 0.003330 & -0.002162 \\
\hline C & -0.003354 & 0.007734 & 1.517908 \\
\hline C & 1.257160 & -0.011985 & 2.082484 \\
\hline$S$ & 2.496211 & -0.071359 & 0.814782 \\
\hline$N$ & 1.370651 & -0.398680 & -0.368831 \\
\hline C & 1.467088 & 0.041983 & 3.455501 \\
\hline C & 0.337931 & 0.104084 & 4.273452 \\
\hline C & -0.944105 & 0.119990 & 3.715069 \\
\hline C & -1.123468 & 0.077342 & 2.337865 \\
\hline $\mathrm{Cl}$ & 3.069603 & 0.036962 & 4.097838 \\
\hline $\mathrm{Cl}$ & 0.503025 & 0.163846 & 5.998680 \\
\hline $\mathrm{O}$ & 3.411727 & -1.195541 & 0.962373 \\
\hline O & 3.103622 & 1.250245 & 0.638107 \\
\hline C & 1.824443 & -0.179617 & -1.739350 \\
\hline $\mathrm{N}$ & -0.956722 & -0.942462 & -0.542165 \\
\hline C & -1.009080 & -2.263835 & -0.177689 \\
\hline $\mathrm{O}$ & -0.339627 & -2.712969 & 0.740100 \\
\hline C & -0.371903 & 1.395665 & -0.526119 \\
\hline C & -1.943804 & -3.113809 & -1.004730 \\
\hline $\mathrm{H}$ & -1.802062 & 0.164359 & 4.378614 \\
\hline 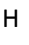 & -2.123512 & 0.082352 & 1.913534 \\
\hline y & 1.963620 & 0.881020 & -1.977791 \\
\hline H & 2.766465 & -0.712756 & -1.890281 \\
\hline $\mathrm{H}$ & 1.079786 & -0.615455 & -2.410661 \\
\hline $\mathrm{H}$ & -2.787201 & -2.542982 & -1.402014 \\
\hline $\mathrm{H}$ & -1.382107 & -3.533032 & -1.847051 \\
\hline $\mathrm{H}$ & -2.311408 & -3.938753 & -0.392355 \\
\hline $\mathrm{H}$ & -0.380338 & 1.405358 & -1.620786 \\
\hline $\mathrm{H}$ & -1.364936 & 1.677812 & -0.164782 \\
\hline H & 0.355879 & 2.130964 & -0.171912 \\
\hline $\mathrm{H}$ & -1.488611 & -0.648083 & -1.353566 \\
\hline
\end{tabular}


$3 a$<smiles>CC(=O)NC1CN(C)S(=O)(=O)c2c1ccc(Cl)c2Cl</smiles>

\section{$E=-2079.7441753$}

C

$-0.028368$

$-0.061538$

146527

S

2.801461

2.504979

09620

1.584545

C

1.486336

0.011984

1.008466

$-0.338193$

$-0.603005$

$-0.268203$

$0.035893 \quad 3.699975$

C

$-1.392656$

0.0573564 .260342

C

$-1.241883$

3.438252

2.397611

$0.033152 \quad 2.062154$

Cl

$-0.492874$

$-0.115546$

4.729838

3.548459

110612

5.979410

O

3.341015

1.191297

2.184087

O

2.366713

$-1.256582$

1.605189

$1.957524-0.253272$

$-0.859324$

$-0.972887$

$-0.613209$

$-1.848573$

$-0.613465$

$-1.478476$

$-2.690581$

$-1.746403$

$-2.013669$

$-2.045215$

0.553353

$-1.804474$

$\begin{array}{lll}-2.381244 & -0.049298 & 3.886226\end{array}$

$\begin{array}{lll}-2.124439 & -0.047567 & 1.429200\end{array}$

$\begin{array}{lll}1.525071 & 2.414629 & 0.282211\end{array}$

$3.289296 \quad 2.465540 \quad 0.022842$

$2.208054 \quad 2.064563 \quad-1.328791$

$-2.558691-1.795006 \quad-3.098752$

$-2.435005-2.715366-1.579036$

$\begin{array}{lll}-3.742791 & -1.525607 & -1.814779\end{array}$

$1.510888-0.171761-1.683129$

$1.746845 \quad-1.384669-0.414896$

$\begin{array}{lll}-0.733407 & -1.955698 & -0.394400\end{array}$

$\mathrm{H}$

$-0.230737$

0.955999

$-0.422020$ 
$4 a$<smiles>CNS(=O)(=O)c1c(C(C)=NC(C)=O)ccc(Cl)c1Cl</smiles>

\section{$E=-2079.7051622$}

\begin{tabular}{|c|c|c|c|}
\hline C & 0.051073 & 0.022270 & -0.000515 \\
\hline C & 0.054284 & 0.021142 & 1.398943 \\
\hline C & 1.271999 & -0.020860 & 2.100203 \\
\hline C & 2.466452 & -0.169688 & 1.389195 \\
\hline C & 2.458002 & -0.246313 & 0.000523 \\
\hline C & 1.259266 & -0.133555 & -0.684386 \\
\hline$S$ & -1.496732 & 0.091442 & 2.332092 \\
\hline 0 & -1.386520 & -0.866013 & 3.423723 \\
\hline $\mathrm{Cl}$ & 1.343107 & 0.176326 & 3.818404 \\
\hline $\mathrm{Cl}$ & 3.992188 & -0.252119 & 2.211225 \\
\hline C & -1.156350 & 0.258193 & -0.867097 \\
\hline$N$ & -1.550475 & 1.460634 & -0.984463 \\
\hline C & -2.590239 & 1.809144 & -1.860812 \\
\hline C & -3.932479 & 1.965216 & -1.197718 \\
\hline C & -1.677847 & -0.921098 & -1.642034 \\
\hline 0 & -1.804439 & 1.491872 & 2.595754 \\
\hline$N$ & -2.611759 & -0.469016 & 1.254513 \\
\hline C & -3.032316 & -1.870292 & 1.355196 \\
\hline $\mathrm{O}$ & -2.381778 & 2.026663 & -3.037584 \\
\hline $\mathrm{H}$ & 3.393434 & -0.364711 & -0.536724 \\
\hline $\mathrm{H}$ & 1.259856 & -0.143040 & -1.770761 \\
\hline $\mathrm{H}$ & -2.160669 & -2.520707 & 1.252057 \\
\hline $\mathrm{H}$ & -3.538188 & -2.091299 & 2.298860 \\
\hline $\mathrm{H}$ & -3.709242 & -2.062595 & 0.520197 \\
\hline $\mathrm{H}$ & -4.295695 & 0.974258 & -0.896444 \\
\hline $\mathrm{H}$ & -3.841729 & 2.578143 & -0.295874 \\
\hline $\mathrm{H}$ & -4.644270 & 2.414157 & -1.892140 \\
\hline $\mathrm{H}$ & -2.720582 & -0.776876 & -1.939351 \\
\hline $\mathrm{H}$ & -1.078376 & -1.044041 & -2.552726 \\
\hline $\mathrm{H}$ & -1.587775 & -1.838972 & -1.056535 \\
\hline $\mathrm{H}$ & -3.365876 & 0.209412 & 1.151355 \\
\hline
\end{tabular}


$5 a$<smiles>C=C(NC(C)=O)c1ccc(Cl)c(Cl)c1S(=O)(=O)NC</smiles>

\section{$E=-2079.7148145$}

C

$\begin{array}{lll}-0.006744 & -0.002027 & 0.015107\end{array}$

$\begin{array}{llll}\text { C } & 1.240812 & -0.008368 & 2.087621\end{array}$

$\begin{array}{llll}\text { C } & 2.431502 & 0.148238 & 1.359498\end{array}$

$\begin{array}{llll}\text { C } & 2.384472 & 0.165996 & -0.041326\end{array}$

$\begin{array}{llll}\text { C } & 1.172820 & 0.057508 & -0.711108\end{array}$

$\begin{array}{llll}\text { C } & -1.313238 & 0.062193 & 2.107641\end{array}$

C $\quad-2.289261 \quad-0.828356 \quad 1.913634$

S $\quad 1.313514 \quad-0.311240 \quad 3.893241$

$\begin{array}{llll}\mathrm{N} & 0.064402 & -1.283609 & 4.286348\end{array}$

$\begin{array}{llll}C & 0.010959 & -2.634584 & 3.728212\end{array}$

$\begin{array}{llll}\mathrm{Cl} & 3.959865 & 0.408293 & 2.130528\end{array}$

$\begin{array}{llll}\mathrm{Cl} & 3.831022 & 0.338916 & -0.984590\end{array}$

O $2.513549 \quad-1.101706 \quad 4.119560$

$\begin{array}{llll}\text { O } & 1.115072 & 0.959322 & 4.581348\end{array}$

$\begin{array}{llll}\mathrm{N} & -1.437538 & 1.194388 & 2.947644\end{array}$

$\begin{array}{llll}\text { C } & -2.020416 & 1.222985 & 4.186373\end{array}$

$\begin{array}{llll}\text { O } & -2.513166 & 0.221252 & 4.699019\end{array}$

$\begin{array}{llll}\text { C } & -1.956793 & 2.547039 & 4.897802\end{array}$

$\begin{array}{llll}\text { H } & 1.155778 & 0.065714 & -1.796059\end{array}$

$\begin{array}{llll}\text { H } & -0.960732 & -0.010306 & -0.502935\end{array}$

$\begin{array}{llll}H & -0.038538 & -2.637504 & 2.631914\end{array}$

$\begin{array}{llll}\text { H } & 0.883734 & -3.200769 & 4.056698\end{array}$

$\begin{array}{llll}\text { H } & -0.890252 & -3.107041 & 4.122959\end{array}$

$\begin{array}{llll}\text { H } & -2.903555 & 2.716916 & 5.415055\end{array}$

$\begin{array}{llll}\text { H } & -1.161465 & 2.490681 & 5.648638\end{array}$

$\begin{array}{llll}\text { H } & -1.748555 & 3.379956 & 4.223022\end{array}$

$\begin{array}{llll}\text { H } & -3.256963 & -0.723114 & 2.391021\end{array}$

$\begin{array}{llll}\text { H } & -2.113675 & -1.695658 & 1.285078\end{array}$

$\begin{array}{llll}\text { H } & -0.914494 & 2.020761 & 2.674337\end{array}$

$\begin{array}{llll}\text { H } & -0.837874 & -0.814821 & 4.395092\end{array}$ 
<smiles>CC(=O)N1C(C)c2ccc(Cl)c(Cl)c2S(=O)(=O)N1C</smiles>

\section{$E=-2079.1997389$}

\begin{tabular}{|c|c|c|c|}
\hline C & -0.938962 & 2.348753 & 0.159922 \\
\hline C & 0.031347 & 1.271298 & 0.061767 \\
\hline C & -0.567407 & -0.063916 & -0.008676 \\
\hline C & -1.960216 & -0.244046 & -0.104158 \\
\hline C & -2.824876 & 0.830670 & -0.050099 \\
\hline C & -2.283184 & 2.135108 & 0.103789 \\
\hline$S$ & 0.536452 & -1.416574 & 0.027914 \\
\hline$N$ & 1.886218 & -0.717590 & 0.822087 \\
\hline$N$ & 2.276662 & 0.420787 & 0.112428 \\
\hline C & 1.377020 & 1.534318 & 0.103720 \\
\hline C & 1.967271 & 2.896086 & 0.345695 \\
\hline 0 & 0.091067 & -2.507677 & 0.895782 \\
\hline $\mathrm{O}$ & 1.024702 & -1.759487 & -1.309911 \\
\hline C & 1.691125 & -0.470248 & 2.255800 \\
\hline C & 3.386943 & 0.325235 & -0.695913 \\
\hline $\mathrm{O}$ & 3.676594 & 1.231819 & -1.468644 \\
\hline $\mathrm{Cl}$ & -2.617279 & -1.842538 & -0.354211 \\
\hline $\mathrm{Cl}$ & -4.556987 & 0.661178 & -0.215656 \\
\hline C & 4.238285 & -0.913127 & -0.540564 \\
\hline $\mathrm{H}$ & -2.968523 & 2.976827 & 0.161889 \\
\hline $\mathrm{H}$ & -0.573495 & 3.366507 & 0.253622 \\
\hline $\mathrm{H}$ & 0.887368 & 0.249540 & 2.455302 \\
\hline $\mathrm{H}$ & 1.467015 & -1.420631 & 2.740059 \\
\hline $\mathrm{H}$ & 2.635881 & -0.079465 & 2.641859 \\
\hline $\mathrm{H}$ & 4.472642 & -1.108961 & 0.509573 \\
\hline $\mathrm{H}$ & 3.709118 & -1.787019 & -0.930499 \\
\hline H & 5.159021 & -0.759548 & -1.105730 \\
\hline $\mathrm{H}$ & 1.491001 & 3.404717 & 1.197005 \\
\hline $\mathrm{H}$ & 3.032148 & 2.802925 & 0.574216 \\
\hline $\mathrm{H}$ & 1.883965 & 3.562990 & -0.523289 \\
\hline
\end{tabular}




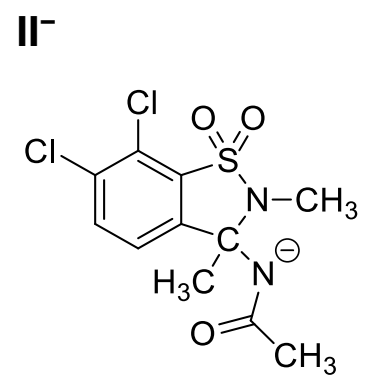

\section{$E=-2079.2467176$}

\begin{tabular}{|c|c|c|c|}
\hline C & -1.755323 & -0.212508 & 0.785915 \\
\hline C & -0.312557 & -0.659415 & 0.582195 \\
\hline C & 0.541442 & 0.315563 & 0.099156 \\
\hline$S$ & -0.355118 & 1.820720 & -0.185627 \\
\hline $\mathrm{N}$ & -1.816846 & 1.071100 & -0.010607 \\
\hline C & 1.905484 & 0.106027 & -0.075248 \\
\hline C & 2.408988 & -1.152542 & 0.248667 \\
\hline C & 1.560663 & -2.149952 & 0.742025 \\
\hline C & 0.205320 & -1.907762 & 0.917814 \\
\hline $\mathrm{Cl}$ & 2.925657 & 1.377236 & -0.660660 \\
\hline $\mathrm{Cl}$ & 4.100698 & -1.509641 & 0.052066 \\
\hline $\mathrm{O}$ & -0.196514 & 2.337025 & -1.545173 \\
\hline $\mathrm{O}$ & -0.070601 & 2.781662 & 0.891142 \\
\hline C & -2.950602 & 1.960501 & 0.207704 \\
\hline$N$ & -2.771775 & -1.132101 & 0.372091 \\
\hline C & -2.605517 & -1.690693 & -0.831825 \\
\hline O & -1.652313 & -1.527226 & -1.628830 \\
\hline C & -1.953703 & 0.084374 & 2.279660 \\
\hline C & -3.721210 & -2.641983 & -1.251600 \\
\hline $\mathrm{H}$ & 1.982257 & -3.121452 & 0.982609 \\
\hline $\mathrm{H}$ & -0.450650 & -2.689339 & 1.291035 \\
\hline $\mathrm{H}$ & -2.904967 & 2.485450 & 1.170597 \\
\hline $\mathrm{H}$ & -2.998258 & 2.694831 & -0.601505 \\
\hline $\mathrm{H}$ & -3.853266 & 1.346341 & 0.168976 \\
\hline $\mathrm{H}$ & -4.493579 & -2.744756 & -0.485324 \\
\hline H & -4.179151 & -2.273414 & -2.176629 \\
\hline $\mathrm{H}$ & -3.295390 & -3.627708 & -1.471226 \\
\hline $\mathrm{H}$ & -2.978954 & 0.426604 & 2.452675 \\
\hline $\mathrm{H}$ & -1.804806 & -0.836772 & 2.851922 \\
\hline $\mathrm{H}$ & -1.251509 & 0.845222 & 2.638741 \\
\hline
\end{tabular}




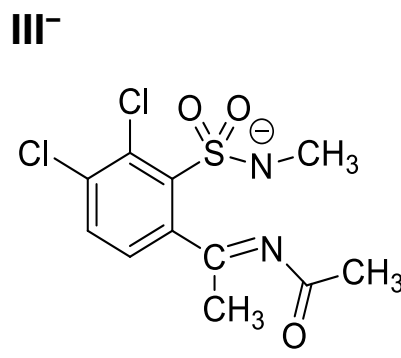

\section{$E=-2079.2208202$}

$\begin{array}{llll}\text { C } & 1.974512 & -0.011257 & -0.171359 \\ \text { C } & 2.573882 & -1.251161 & 0.081773 \\ \text { C } & 1.809945 & -2.343657 & 0.474854 \\ \text { C } & 0.433882 & -2.205841 & 0.576308 \\ \text { C } & -0.178898 & -0.975634 & 0.329986 \\ \text { C } & 0.592767 & 0.149099 & 0.007835 \\ \text { Cl } & 4.293040 & -1.480884 & -0.093833 \\ \text { C } & -1.683548 & -0.975034 & 0.349908 \\ \text { N } & -2.268504 & -1.078459 & -0.776133 \\ \mathrm{C} & -3.659968 & -1.152519 & -0.874720 \\ \mathrm{C} & -4.321763 & 0.175468 & -1.130183 \\ \mathrm{~S} & -0.270355 & 1.785288 & -0.122581 \\ \mathrm{~N} & -1.536710 & 1.675668 & 0.789315 \\ \mathrm{C} & -1.270907 & 1.936796 & 2.201859 \\ \mathrm{Cl} & 2.973125 & 1.277525 & -0.775258 \\ \mathrm{C} & -2.376173 & -1.082641 & 1.682841 \\ \mathrm{O} & 0.729732 & 2.758579 & 0.363409 \\ \mathrm{O} & -0.640376 & 1.873983 & -1.545516 \\ \mathrm{O} & -4.255119 & -2.215289 & -0.850256 \\ \mathrm{H} & -2.291562 & -3.296728 & 0.669020 \\ \mathrm{H} & -0.174820 & -3.068976 & 0.833476 \\ \mathrm{H} & -0.528554 & 1.250676 & 2.647418 \\ \mathrm{H} & -0.915319 & 2.959320 & 2.387544 \\ \mathrm{H} & -2.209876 & 1.800606 & 2.749041 \\ \mathrm{H} & -3.982568 & 0.910482 & -0.392096 \\ \mathrm{H} & -4.008942 & 0.544723 & -2.113371 \\ \mathrm{H} & -5.407365 & 0.066540 & -1.104191 \\ \mathrm{H} & -3.345587 & -0.574546 & 1.660291 \\ \mathrm{H} & -2.551095 & -2.142238 & 1.909757 \\ \mathrm{H} & -0.657744 & 2.480966\end{array}$




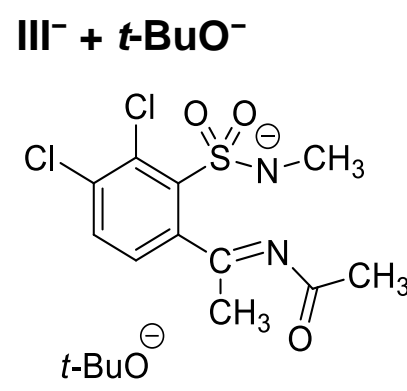

\section{$E=-2312.2586146$}

\begin{tabular}{|c|c|c|c|}
\hline C & 0.000000 & 0.000000 & 0.000000 \\
\hline C & 0.000000 & 0.000000 & 1.402951 \\
\hline$C$ & 1.230813 & 0.000000 & 2.073928 \\
\hline C & 2.421304 & -0.119687 & 1.348228 \\
\hline C & 2.398605 & -0.251267 & -0.034874 \\
\hline C & 1.189653 & -0.180029 & -0.714442 \\
\hline$S$ & -1.618816 & -0.174889 & 2.292903 \\
\hline $\mathrm{O}$ & -1.277873 & -1.055977 & 3.432982 \\
\hline $\mathrm{Cl}$ & 1.329830 & 0.224930 & 3.797975 \\
\hline $\mathrm{Cl}$ & 3.975268 & -0.125826 & 2.145893 \\
\hline C & -1.214244 & 0.316785 & -0.829552 \\
\hline $\mathrm{N}$ & -1.667537 & 1.505114 & -0.735171 \\
\hline C & -2.747461 & 1.964986 & -1.483901 \\
\hline C & -4.095661 & 1.723067 & -0.852472 \\
\hline C & -1.637239 & -0.705351 & -1.839673 \\
\hline 0 & -2.028767 & 1.198709 & 2.634393 \\
\hline $\mathrm{N}$ & -2.610986 & -0.828175 & 1.274707 \\
\hline C & -2.440947 & -2.270591 & 1.128137 \\
\hline 0 & -2.597399 & 2.598873 & -2.514808 \\
\hline 0 & 0.964632 & -1.086270 & -3.669843 \\
\hline C & 1.398565 & -2.372708 & -3.802263 \\
\hline C & 2.820690 & -2.553934 & -3.213280 \\
\hline C & 0.463027 & -3.366159 & -3.065682 \\
\hline C & 1.453925 & -2.797394 & -5.290484 \\
\hline $\mathrm{H}$ & 3.330782 & -0.381542 & -0.577120 \\
\hline $\mathrm{H}$ & 1.168582 & -0.295529 & -1.808509 \\
\hline $\mathrm{H}$ & -1.440116 & -2.561550 & 0.759153 \\
\hline $\mathrm{H}$ & -2.612899 & -2.822009 & 2.063281 \\
\hline $\mathrm{H}$ & -3.171924 & -2.620508 & 0.391142 \\
\hline $\mathrm{H}$ & -4.252249 & 0.647165 & -0.719405 \\
\hline $\mathrm{H}$ & -4.120576 & 2.173099 & 0.145217 \\
\hline $\mathrm{H}$ & -4.882578 & 2.149460 & -1.477409 \\
\hline $\mathrm{H}$ & -2.558118 & -0.411822 & -2.353143 \\
\hline H & -0.823988 & -0.830746 & -2.582726 \\
\hline
\end{tabular}




$\begin{array}{llll}\mathrm{H} & -1.786932 & -1.672902 & -1.351978 \\ \mathrm{H} & 3.217508 & -3.572936 & -3.336875 \\ \mathrm{H} & 3.509886 & -1.852079 & -3.701155 \\ \mathrm{H} & 2.808997 & -2.322955 & -2.140095 \\ \mathrm{H} & 0.785875 & -4.414155 & -3.155254 \\ \mathrm{H} & 0.418151 & -3.111976 & -1.997360 \\ \mathrm{H} & -0.554976 & -3.284481 & -3.470819 \\ \mathrm{H} & 1.791376 & -3.834574 & -5.437282 \\ \mathrm{H} & 0.457684 & -2.691597 & -5.739648 \\ \mathrm{H} & 2.137431 & -2.132318 & -5.834556\end{array}$

\section{$\mathrm{IV}^{2-}+t-\mathrm{BuOH}$}<smiles>C=C(NC(C)=O)c1ccc(Cl)c(Cl)c1S(=O)(=O)OC</smiles>

\section{$E=-2312.2910731$}

C

C

C

C

C

c

C
C

C

(n)

a

$\circ$$$
\text { a }
$$

$\begin{array}{lll}-0.006208 & -0.015598 & -0.002726 \\ -0.003136 & -0.004534 & 1.397389 \\ 1.217584 & 0.005155 & 2.087571 \\ 2.406708 & -0.178793 & 1.354612 \\ 2.374888 & -0.210188 & -0.041576 \\ 1.169952 & -0.092929 & -0.728041 \\ -1.351316 & -0.117483 & 2.056446 \\ -1.600807 & -1.299588 & 2.671097 \\ 1.292764 & 0.438445 & 3.901766 \\ 0.050317 & 1.276478 & 4.306748 \\ -1.031076 & 0.670133 & 5.065417 \\ 3.939366 & -0.456725 & 2.136960 \\ 3.832957 & -0.404002 & -0.987562 \\ 1.447073 & -0.869143 & 4.591669 \\ 2.484759 & 1.314595 & 3.908083 \\ -2.165027 & 0.980986 & 1.828854 \\ -3.447826 & 0.978713 & 2.234736 \\ -4.077860 & 0.051843 & 2.788854 \\ -4.187972 & 2.291742 & 1.999549 \\ -1.372569 & 3.233895 & 0.361335\end{array}$




\begin{tabular}{|c|c|c|c|}
\hline C & -0.591392 & 4.153815 & 1.112520 \\
\hline C & -1.306239 & 4.513942 & 2.418694 \\
\hline C & 0.776798 & 3.547644 & 1.429122 \\
\hline C & -0.436017 & 5.391499 & 0.233945 \\
\hline $\mathrm{H}$ & 1.163382 & -0.101450 & -1.813482 \\
\hline $\mathrm{H}$ & -0.960884 & 0.022997 & -0.520588 \\
\hline $\mathrm{H}$ & -0.847658 & -0.381317 & 5.323027 \\
\hline $\mathrm{H}$ & -1.161193 & 1.225174 & 6.006298 \\
\hline $\mathrm{H}$ & -1.978780 & 0.722255 & 4.514125 \\
\hline $\mathrm{H}$ & -5.208793 & 2.081808 & 1.664114 \\
\hline $\mathrm{H}$ & -4.259373 & 2.830260 & 2.953496 \\
\hline $\mathrm{H}$ & -3.681305 & 2.935215 & 1.277074 \\
\hline $\mathrm{H}$ & -2.556202 & -1.520385 & 3.125784 \\
\hline $\mathrm{H}$ & -0.799996 & -2.031498 & 2.745516 \\
\hline $\mathrm{H}$ & -1.569055 & 2.424807 & 0.915911 \\
\hline $\mathrm{H}$ & -0.719974 & 5.236435 & 2.999771 \\
\hline $\mathrm{H}$ & -2.289700 & 4.952101 & 2.209983 \\
\hline $\mathrm{H}$ & -1.442732 & 3.617272 & 3.035328 \\
\hline $\mathrm{H}$ & 1.432085 & 4.279009 & 1.918631 \\
\hline $\mathrm{H}$ & 0.665423 & 2.695330 & 2.107485 \\
\hline H & 1.262581 & 3.204242 & 0.507174 \\
\hline $\mathrm{H}$ & 0.153437 & 6.163019 & 0.742468 \\
\hline $\mathrm{H}$ & 0.069618 & 5.130516 & -0.703231 \\
\hline $\mathrm{H}$ & -1.419058 & 5.810199 & -0.011833 \\
\hline
\end{tabular}

$\mathbf{V}^{-}$<smiles>C=C(NC(C)=O)c1ccc(Cl)c(Cl)c1S(=O)(=O)NC</smiles>

\section{$E=-2079.2256846$}

$\begin{array}{llll}\text { C } & -0.547124 & -0.048543 & 0.070327 \\ C & -1.934466 & -0.151709 & -0.124605 \\ C & -2.740448 & 0.993965 & -0.100681 \\ C & -2.178381 & 2.251042 & 0.073365 \\ C & -0.801787 & 2.364416 & 0.175278 \\ C & 0.027683 & 1.236764 & 0.166401\end{array}$




$\begin{array}{llll}\mathrm{Cl} & -2.710340 & -1.667329 & -0.481587 \\ \mathrm{Cl} & -4.468865 & 0.898601 & -0.306739 \\ \mathrm{C} & 1.501888 & 1.509244 & 0.190862 \\ \mathrm{C} & 2.027754 & 2.329002 & 1.114474 \\ \mathrm{~S} & 0.541644 & -1.562117 & 0.226902 \\ \mathrm{O} & 0.925652 & -1.811313 & -1.183562 \\ \mathrm{~N} & 2.201115 & 0.921323 & -0.876178 \\ \mathrm{C} & 3.546790 & 0.633617 & -0.871211 \\ \mathrm{O} & 4.352819 & 1.193549 & -0.142072 \\ \mathrm{C} & 3.934584 & -0.444052 & -1.853725 \\ \mathrm{~N} & 1.793719 & -1.172678 & 1.056615 \\ \mathrm{C} & 1.551768 & -0.982059 & 2.481560 \\ \mathrm{O} & -0.334227 & -2.565929 & 0.861766 \\ \mathrm{H} & -2.813081 & 3.131210 & 0.087262 \\ \mathrm{H} & -0.350255 & 3.349261 & 0.242909 \\ \mathrm{H} & 0.794900 & -0.205420 & 2.692567 \\ \mathrm{H} & 1.232628 & -1.900562 & 2.995111 \\ \mathrm{H} & 2.489739 & -0.645458 & 2.934921 \\ \mathrm{H} & 5.019495 & -0.464530 & -1.964704 \\ \mathrm{H} & 3.580133 & -1.406275 & -1.467256 \\ \mathrm{H} & 3.463802 & -0.283941 & -2.829007 \\ \mathrm{H} & 3.075346 & 2.594347 & 1.120555 \\ \mathrm{H} & 1.384668 & 2.711413 & 1.901069 \\ \mathrm{H} & 1.655450 & 0.261110 & -1.426706\end{array}$

$\mathrm{VI}^{-}$<smiles>CC(=O)NC1Cc2ccc(Cl)c(Cl)c2S(=O)(=O)N(C)C1</smiles>

\section{$E=-2079.2316795$}

$\begin{array}{llll}\text { C } & 1.753746 & 0.144467 & -0.466960 \\ \text { C } & 0.482244 & 0.615648 & -0.241411 \\ \text { C } & -0.699675 & -0.236307 & -0.121804 \\ \text { S } & -0.417660 & -1.967889 & -0.277121 \\ \text { N } & 1.159780 & -2.131299 & 0.266813 \\ \text { C } & 2.085504 & -1.319262 & -0.544309\end{array}$




$\begin{array}{llll}\text { C } & -1.988182 & 0.308355 & -0.003083 \\ \text { C } & -2.182595 & 1.675481 & 0.105864 \\ \text { C } & -1.049957 & 2.525730 & 0.082363 \\ \text { C } & 0.211248 & 2.033496 & -0.084541 \\ \text { Cl } & -3.389924 & -0.731958 & -0.052256 \\ \text { Cl } & -3.768834 & 2.398730 & 0.248306 \\ \text { O } & -1.254481 & -2.746202 & 0.636092 \\ \text { O } & -0.397816 & -2.353185 & -1.692219 \\ \text { C } & 1.303919 & -1.925073 & 1.713797 \\ \mathrm{~N} & 2.831554 & 1.055651 & -0.661581 \\ \mathrm{C} & 3.936731 & 1.116223 & 0.125038 \\ \mathrm{C} & 5.015693 & 2.074151 & -0.333108 \\ \mathrm{O} & 4.081331 & 0.425432 & 1.134287 \\ \mathrm{H} & -1.201938 & 3.596083 & 0.198107 \\ \mathrm{H} & 1.053072 & 2.718990 & -0.093602 \\ \mathrm{H} & 1.056794 & -0.901371 & 2.024415 \\ \mathrm{H} & 0.665703 & -2.634261 & 2.241616 \\ \mathrm{H} & 2.347933 & -2.125077 & 1.965831 \\ \mathrm{H} & 5.882310 & 1.496523 & -0.671331 \\ \mathrm{H} & 4.691782 & 2.730519 & -1.145326 \\ \mathrm{H} & 5.330935 & 2.681273 & 0.519610 \\ \mathrm{H} & 3.087395 & -1.501002 & -0.141394 \\ \mathrm{H} & 2.061959 & -1.720113 & -1.567245 \\ \mathrm{H} & 2.821808 & 1.653248 & -1.482603 \\ & & & \\ & & & \\ & & & \end{array}$

$\mathrm{VII}^{-}$<smiles>CC(=O)N[C@@H]1CN(C)S(=O)(=O)c2c1ccc(Cl)c2Cl</smiles>

\section{$E=-2079.2436737$}

$\begin{array}{llll}\text { C } & 1.982904 & -0.308673 & 0.060434 \\ \mathrm{C} & 0.623929 & 0.396849 & 0.116976 \\ \mathrm{C} & -0.623083 & -0.230272 & -0.029549 \\ \mathrm{~S} & -0.700344 & -2.020824 & -0.229841 \\ \mathrm{~N} & 0.820631 & -2.512972 & 0.186693 \\ \mathrm{C} & 1.857856 & -1.722499 & -0.499365\end{array}$




\begin{tabular}{llll} 
C & -1.817594 & 0.510779 & -0.046509 \\
C & -1.768581 & 1.887039 & 0.154020 \\
C & -0.541452 & 2.520094 & 0.348889 \\
C & 0.629527 & 1.784463 & 0.316535 \\
Cl & -3.345239 & -0.258578 & -0.343621 \\
Cl & -3.215831 & 2.850802 & 0.162718 \\
O & -1.616468 & -2.587691 & 0.753010 \\
O & -0.905531 & -2.298988 & -1.647232 \\
C & 1.042176 & -2.726138 & 1.624813 \\
N & 2.936325 & 0.410692 & -0.761157 \\
$\mathrm{C}$ & 3.771504 & 1.196486 & -0.092230 \\
$\mathrm{C}$ & 4.829099 & 1.890596 & -0.940644 \\
$\mathrm{O}$ & 3.777254 & 1.434866 & 1.148949 \\
$\mathrm{H}$ & -0.516903 & 3.593294 & 0.511902 \\
$\mathrm{H}$ & 1.585173 & 2.280246 & 0.455165 \\
$\mathrm{H}$ & 2.339128 & -0.362793 & 1.101900 \\
$\mathrm{H}$ & 0.869526 & -1.825119 & 2.227048 \\
$\mathrm{H}$ & 0.392276 & -3.526263 & 1.977562 \\
$\mathrm{H}$ & 2.084247 & -3.034431 & 1.739084 \\
$\mathrm{H}$ & 4.740257 & 2.976450 & -0.819692 \\
$\mathrm{H}$ & 5.825494 & 1.605475 & -0.583605 \\
$\mathrm{H}$ & 4.740242 & 1.637325 & -2.000305 \\
$\mathrm{H}$ & 2.806710 & -2.254354 & -0.386339 \\
$\mathrm{H}$ & 1.619098 & -1.686336 & -1.566697 \\
& & & \\
\hline & & & \\
& & &
\end{tabular}

\section{$\mathrm{VIII}^{2-}+t-\mathrm{BuOH}$}<smiles></smiles>

\section{$E=-2312.2910731$}

$\begin{array}{llll}C & -0.006208 & -0.015598 & -0.002726 \\ C & -0.003136 & -0.004534 & 1.397389 \\ C & 1.217584 & 0.005155 & 2.087571 \\ C & 2.406708 & -0.178793 & 1.354612 \\ C & 2.374888 & -0.210188 & -0.041576 \\ C & 1.169952 & -0.092929 & -0.728041\end{array}$




\begin{tabular}{|c|c|c|c|}
\hline & -1.351316 & -0.117483 & 2.056446 \\
\hline C & -1.600807 & -1.299588 & 2.671097 \\
\hline 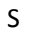 & 1.292764 & 0.438445 & 3.901766 \\
\hline$N$ & 0.050317 & 1.276478 & 4.306748 \\
\hline$C$ & -1.031076 & 0.670133 & 5.065417 \\
\hline $\mathrm{Cl}$ & 3.939366 & -0.456725 & 2.136960 \\
\hline $\mathrm{Cl}$ & 3.832957 & -0.404002 & -0.987562 \\
\hline & 1.447073 & -0.869143 & 4.591669 \\
\hline 0 & 2.484759 & 1.314595 & 3.908083 \\
\hline $\mathrm{N}$ & -2.165027 & 0.980986 & 1.828854 \\
\hline$C$ & -3.447826 & 0.978713 & 2.234736 \\
\hline o & -4.077860 & 0.051843 & 2.788854 \\
\hline C & -4.187972 & 2.291742 & 1.999549 \\
\hline O & -1.372569 & 3.233895 & 0.361335 \\
\hline C & -0.591392 & 4.153815 & 1.112520 \\
\hline C & -1.306239 & 4.513942 & 2.418694 \\
\hline$C$ & 0.776798 & 3.547644 & 1.429122 \\
\hline C & -0.436017 & 5.391499 & 0.233945 \\
\hline $\mathrm{H}$ & 1.163382 & -0.101450 & -1.813482 \\
\hline $\mathrm{H}$ & -0.960884 & 0.022997 & -0.520588 \\
\hline $\mathrm{H}$ & -0.847658 & -0.381317 & 5.323027 \\
\hline $\mathrm{H}$ & -1.161193 & 1.225174 & 6.006298 \\
\hline 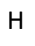 & -1.978780 & 0.722255 & 4.514125 \\
\hline $\mathrm{H}$ & -5.208793 & 2.081808 & 1.664114 \\
\hline H & -4.259373 & 2.830260 & 2.953496 \\
\hline $\mathrm{H}$ & -3.681305 & 2.935215 & 1.277074 \\
\hline $\mathrm{H}$ & -2.556202 & -1.520385 & 3.125784 \\
\hline $\mathrm{H}$ & -0.799996 & -2.031498 & 2.745516 \\
\hline $\mathrm{H}$ & -1.569055 & 2.424807 & 0.915911 \\
\hline $\mathrm{H}$ & -0.719974 & 5.236435 & 2.999771 \\
\hline H & -2.289700 & 4.952101 & 2.209983 \\
\hline $\mathrm{H}$ & -1.442732 & 3.617272 & 3.035328 \\
\hline $\mathrm{H}$ & 1.432085 & 4.279009 & 1.918631 \\
\hline $\mathrm{H}$ & 0.665423 & 2.695330 & 2.107485 \\
\hline H & 1.262581 & 3.204242 & 0.507174 \\
\hline $\mathrm{H}$ & 0.153437 & 6.163019 & 0.742468 \\
\hline H & 0.069618 & 5.130516 & -0.703231 \\
\hline $\mathrm{H}$ & -1.419058 & 5.810199 & -0.011833 \\
\hline
\end{tabular}




\section{TS1}

\section{$E=-2079.1681609$}

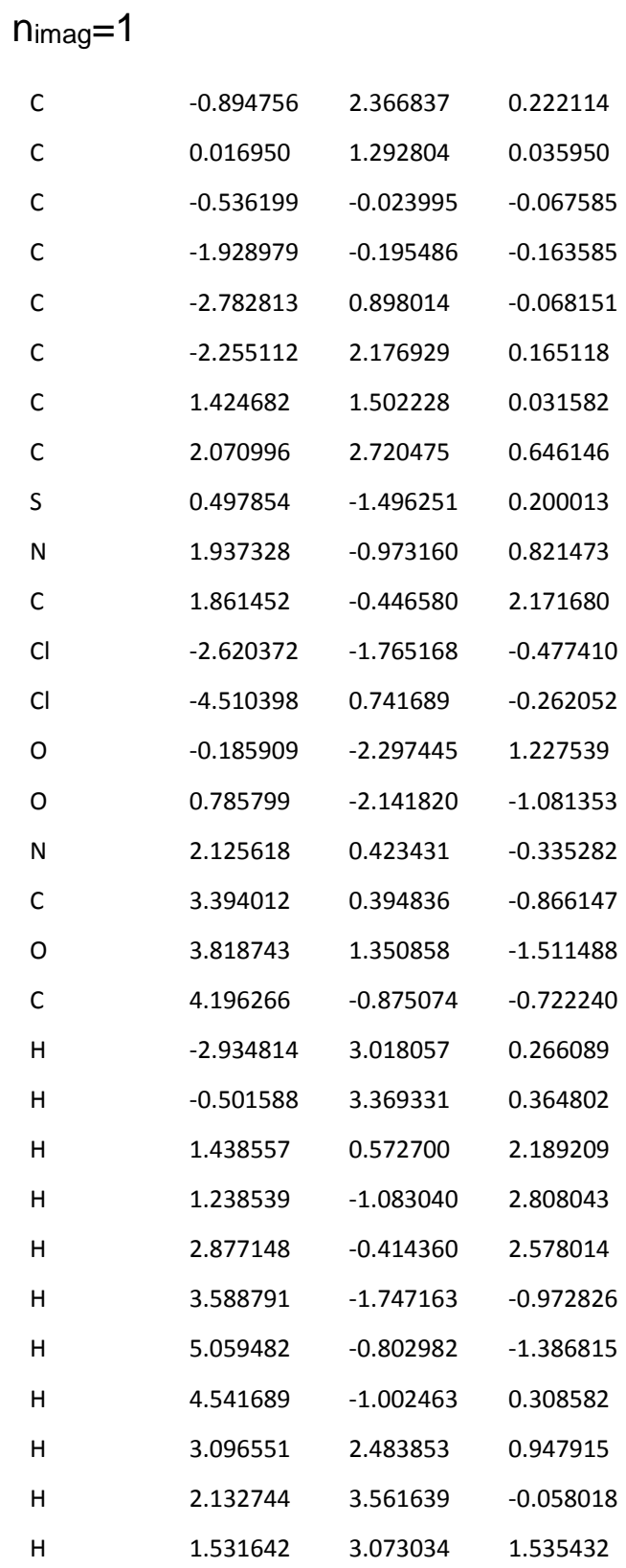




\section{TS2}

\section{$E=-2079,2152398$}

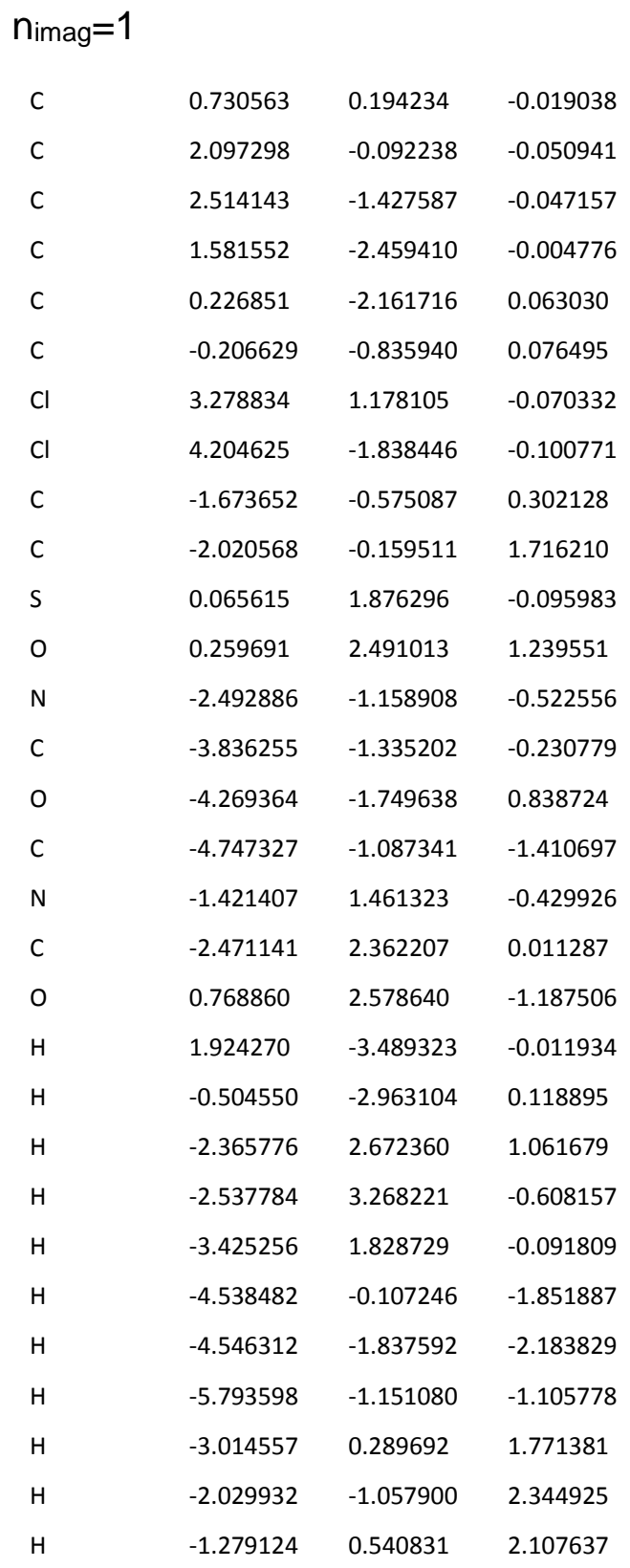




\section{TS3}

$$
E=-2312.2737222
$$

nimag $=1$

$\begin{array}{llll}\text { C } & -1.775077 & 2.333922 & -0.558666\end{array}$

$\begin{array}{llll}\text { C } & -0.405193 & 2.462875 & -0.752647\end{array}$

$\begin{array}{llll}\text { C } & 0.403592 & 1.341692 & -0.645599\end{array}$

$\begin{array}{llll}\text { C } & -0.140834 & 0.096387 & -0.311852\end{array}$

$\begin{array}{llll}\text { C } & -1.508687 & -0.020230 & -0.019920\end{array}$

$\begin{array}{llll}\text { C } & -2.333232 & 1.101894 & -0.202446\end{array}$

$\begin{array}{llll}\text { C } & 0.821808 & -1.062556 & -0.413440\end{array}$

$\begin{array}{llll}\text { C } & 1.975762 & -1.037979 & 0.469227\end{array}$

S $\quad-2.162279 \quad-1.611272 \quad 0.682767$

$\begin{array}{llll}0 & -3.163827 & -1.152514 & 1.674690\end{array}$

$\begin{array}{llll}\mathrm{Cl} & -4.066915 & 0.996607 & -0.073947\end{array}$

$\begin{array}{llll}\mathrm{Cl} & -2.767539 & 3.757283 & -0.775720\end{array}$

N $\quad 0.652846 \quad-1.812496 \quad-1.452691$

$\begin{array}{llll}\mathrm{C} & 1.431614 & -2.928771 & -1.692774\end{array}$

$\begin{array}{llll}\text { O } & 2.267265 & -2.974894 & -2.586728\end{array}$

$\begin{array}{llll}\text { C } & 1.064853 & -4.148814 & -0.875231\end{array}$

$\begin{array}{llll}0 & -2.691984 & -2.340244 & -0.485194\end{array}$

$\begin{array}{llll}\mathrm{N} & -0.956100 & -2.364309 & 1.323061\end{array}$

$\begin{array}{llll}\text { C } & -0.563460 & -1.853040 & 2.631000\end{array}$

$\begin{array}{llll}\text { O } & 3.597564 & 0.830278 & -0.510359\end{array}$

$\begin{array}{llll}\text { C } & 4.276989 & 1.520388 & 0.482771\end{array}$

$\begin{array}{llll}C & 5.288195 & 2.476194 & -0.169262\end{array}$

$\begin{array}{llll}\text { C } & 3.300636 & 2.348732 & 1.343258\end{array}$

$\begin{array}{llll}\text { C } & 5.043520 & 0.547992 & 1.402424\end{array}$

$\begin{array}{llll}\text { H } & 0.013776 & 3.430314 & -1.013236\end{array}$

$\begin{array}{llll}\text { H } & 1.476908 & 1.412981 & -0.829095\end{array}$

$\begin{array}{llll}\text { H } & -0.315141 & -0.776584 & 2.629636\end{array}$

$\begin{array}{llll}H & -1.333117 & -2.005094 & 3.401669\end{array}$

$\begin{array}{llll}H & 0.339606 & -2.389671 & 2.942341\end{array}$

$\begin{array}{llll}H & 1.006754 & -3.893855 & 0.187059\end{array}$

$\begin{array}{llll}\text { H } & 0.063889 & -4.482623 & -1.171815\end{array}$

$\begin{array}{llll}\text { H } & 1.786724 & -4.950144 & -1.047615\end{array}$

$\begin{array}{llll}H & 2.540008 & -1.977332 & 0.481312\end{array}$

$\begin{array}{llll}\mathrm{H} & 2.793436 & -0.113448 & -0.002943\end{array}$

$\begin{array}{llll}\mathrm{H} & 1.725705 & -0.709352 & 1.481949\end{array}$

$\begin{array}{llll}\text { H } & 5.854574 & 3.055778 & 0.573383\end{array}$

$\begin{array}{llll}H & 5.998271 & 1.905261 & -0.780226\end{array}$

$\begin{array}{llll}\mathrm{H} & 4.763289 & 3.177585 & -0.829805\end{array}$ 


$\begin{array}{llll}\mathrm{H} & 3.810865 & 2.893643 & 2.149550 \\ \mathrm{H} & 2.774674 & 3.079377 & 0.715126 \\ \mathrm{H} & 2.546017 & 1.693423 & 1.796678 \\ \mathrm{H} & 5.623277 & 1.072283 & 2.174491 \\ \mathrm{H} & 4.346943 & -0.134390 & 1.905522 \\ \mathrm{H} & 5.733456 & -0.059390 & 0.803188\end{array}$

\section{TS4}

\section{$E=-2079.1863185$}

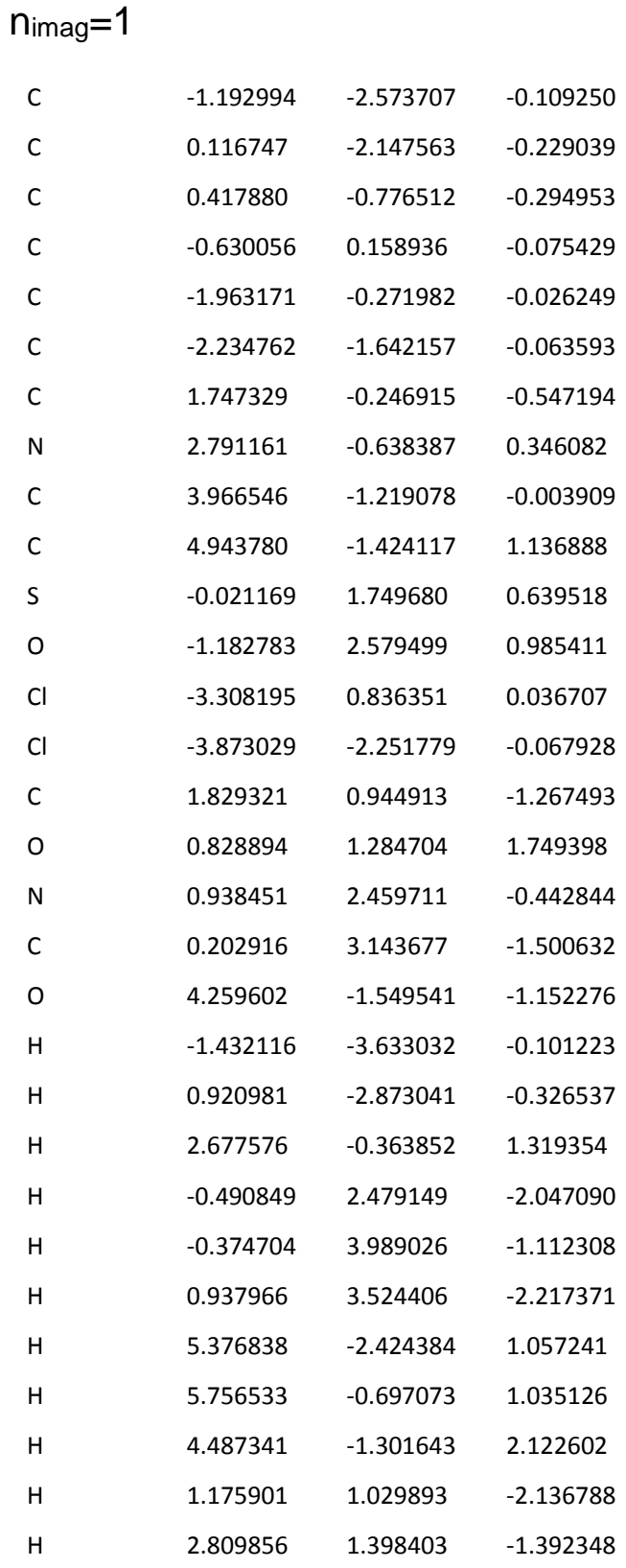




\section{TS5}

\section{$E=-2078.6533059$}

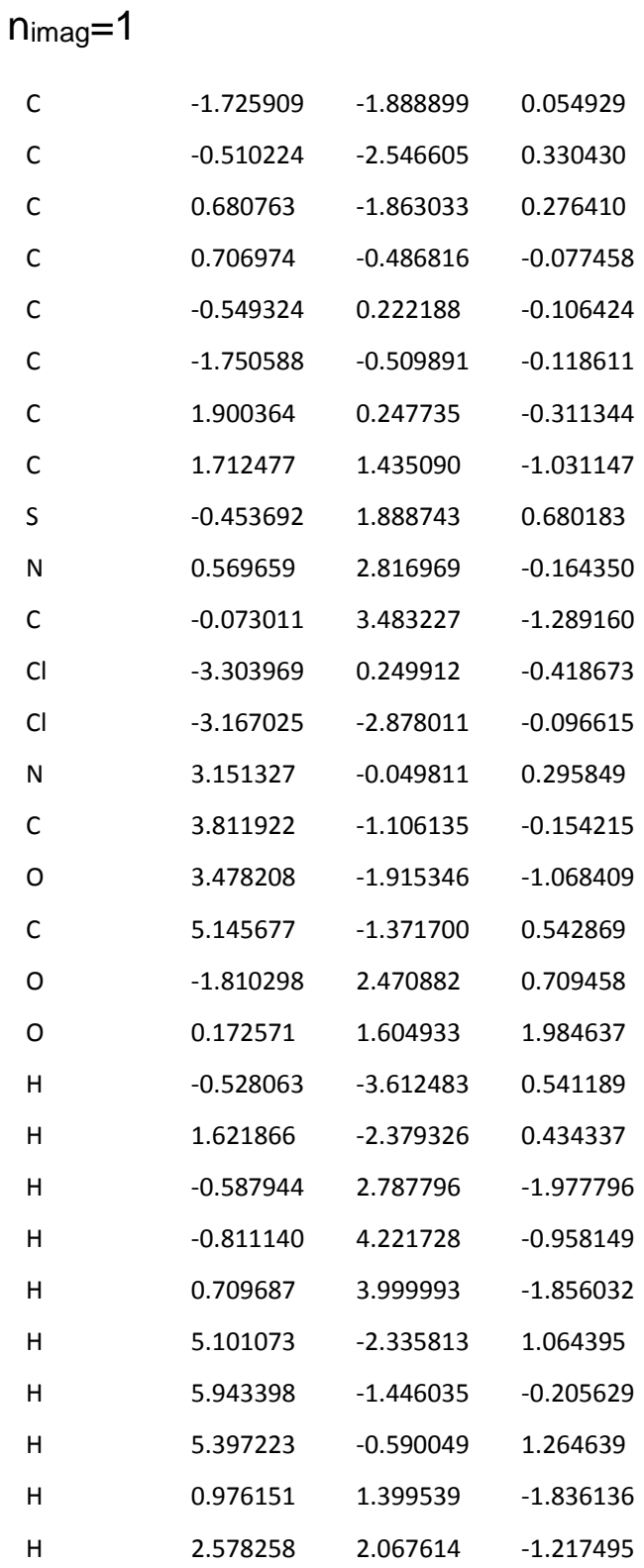


Frequency calculation for gas-phase acidity was performed at M06-2X/6-31+G* using fine grid:

\section{$1 a$}<smiles>CC(=O)N1C(C)c2ccc(Cl)c(Cl)c2S(=O)(=O)N1C</smiles>

\section{$E=-2079.6818009$}

\begin{tabular}{lll}
0.013346 & -0.029703 & -0.006280 \\
0.013098 & 0.007869 & 1.515184 \\
1.162812 & -0.019608 & 2.318090 \\
2.779499 & 0.123104 & 1.538127 \\
2.337985 & 0.796246 & 0.055192 \\
1.367108 & 0.025257 & -0.557114 \\
1.080708 & -0.136163 & 3.714972 \\
-0.175738 & -0.166817 & 4.321975 \\
-1.325410 & -0.102423 & 3.538889 \\
-1.228028 & -0.025851 & 2.160021 \\
2.502391 & -0.285783 & 4.679079 \\
-0.342200 & -0.297332 & 6.038551 \\
3.571109 & 1.139358 & 2.203287 \\
3.293669 & -1.207101 & 1.283778 \\
\hline 2.125399 & 2.250798 & 0.041032 \\
1.692096 & -0.730799 & -1.676018 \\
\hline 3.129765 & -0.681119 & -2.125899 \\
-0.900374 & 1.015949 & -0.661040 \\
0.826281 & -1.382358 & -2.229760 \\
-2.295331 & -0.134154 & 4.024067 \\
-2.139045 & -0.018489 & 1.570519 \\
-0.356508 & -1.019717 & -0.295718 \\
1.313453 & 2.566885 & 0.708419 \\
3.051121 & 2.735613 & 0.347564 \\
1.885285 & 2.522017 & -0.989271 \\
3.448975 & 0.347278 & -2.315776 \\
3.779587 & -1.090653 & -1.347301 \\
3.210347 & -1.273878 & -3.036583 \\
-1.950314 & 0.839038 & -0.414979 \\
-0.642342 & 2.032135 & -0.351441 \\
-0.795314 & 0.931895 & -1.746111 \\
\hline
\end{tabular}


<smiles>CC(=O)N1C(C)c2ccc(Cl)c(Cl)c2S(=O)(=O)N1C</smiles>

\section{$E=-2079.1307768$}

\begin{tabular}{llll} 
C & 0.002314 & 0.009011 & -0.012224 \\
C & 0.005078 & 0.001038 & 1.359275 \\
C & 1.200054 & 0.014535 & 2.206109 \\
S & 2.743479 & 0.072464 & 1.382870 \\
N & 2.278778 & 0.810372 & -0.103414 \\
N & 1.261824 & 0.051934 & -0.689447 \\
C & 1.112388 & -0.018188 & 3.608685 \\
C & -0.112917 & 0.000861 & 4.248822 \\
C & -1.290562 & 0.046216 & 3.461402 \\
C & -1.242866 & 0.048010 & 2.099619 \\
Cl & 2.560559 & -0.142037 & 4.571935 \\
Cl & -0.276754 & -0.083176 & 5.988387 \\
O & 3.688075 & 1.005596 & 1.993709 \\
O & 3.216938 & -1.254834 & 0.998333 \\
C & 1.932722 & 2.228980 & 0.020424 \\
C & 1.603398 & -0.800009 & -1.717566 \\
C & 3.008708 & -0.667658 & -2.267809 \\
C & -1.233518 & 0.129346 & -0.857166 \\
O & 0.790928 & -1.588808 & -2.179489 \\
H & -2.249833 & 0.063394 & 3.972162 \\
H & -2.172668 & 0.054767 & 1.540257 \\
H & 1.026400 & 2.386021 & 0.619294 \\
H & 2.777587 & 2.740401 & 0.481468 \\
H & 1.772472 & 2.608775 & -0.992609 \\
H & 3.264251 & 0.375239 & -2.473247 \\
H & 3.725931 & -1.044290 & -1.533581 \\
\hline & 3.057941 & -1.260943 & -3.182206 \\
H & -1.890803 & 0.942300 & -0.510519 \\
H & -0.952823 & 0.345223 & -1.891133 \\
\hline & -0.795128 & -0.880124 \\
\hline
\end{tabular}


<smiles>CC(=O)N1C(C)c2ccc(Cl)c(Cl)c2S(=O)(=O)N1C</smiles>

\section{$E=-2079.0950682$}

C

C

C

C

C

$\mathrm{Cl}$

$\mathrm{Cl}$

O

O

C

C

C

C

O

H

H

$\mathrm{H}$

H

$\mathrm{H}$

$\mathrm{H}$

H

H

H

H

$\mathrm{H}$

H

\begin{tabular}{|c|c|c|}
\hline 0.019765 & -0.058017 & -0.006569 \\
\hline 0.026905 & 0.024384 & 1.516251 \\
\hline 1.185124 & -0.035368 & 2.315425 \\
\hline .778932 & 0.035252 & 1.534073 \\
\hline 2.354075 & 0.768600 & 0.051811 \\
\hline .369363 & 0.028455 & -0.584374 \\
\hline 1.070461 & -0.100875 & 3.715275 \\
\hline-0.198857 & -0.033008 & 4.285765 \\
\hline-1.391273 & 0.048207 & 3.578246 \\
\hline-1.203988 & 0.055402 & 2.183818 \\
\hline 2.518154 & -0.321992 & 4.676798 \\
\hline-0.279340 & -0.064970 & 6.076442 \\
\hline 3.667320 & 1.000159 & 2.165792 \\
\hline 3.265275 & -1.295418 & 1.197570 \\
\hline 2.086028 & 2.209562 & 0.128684 \\
\hline 1.698535 & -0.690599 & -1.709881 \\
\hline 3.142784 & -0.626679 & -2.151778 \\
\hline-0.912940 & 0.950347 & -0.691536 \\
\hline 0.848832 & -1.336741 & -2.305616 \\
\hline-2.094542 & 0.064250 & 1.551706 \\
\hline-0.326117 & -1.062239 & -0.274179 \\
\hline 1.236404 & 2.445424 & 0.782532 \\
\hline 2.981232 & 2.700149 & 0.509344 \\
\hline 1.875960 & 2.549048 & -0.888984 \\
\hline 3.458343 & 0.406744 & -2.319901 \\
\hline 3.788308 & -1.044412 & -1.374287 \\
\hline 3.229783 & -1.203363 & -3.073062 \\
\hline-1.956688 & 0.753399 & -0.438203 \\
\hline-0.6805 & 1.976515 & -0.392428 \\
\hline .7 & 0.852597 & 775 \\
\hline
\end{tabular}


<smiles>CC(=O)N1C(C)c2ccc(Cl)c(Cl)c2S(=O)(=O)N1C</smiles>

\section{$E=-2079.0943152$}

C

S

N

C

C

C

C

Cl

$\mathrm{Cl}$

O

O

C

C

C

C

O

H

$\mathrm{H}$

$\mathrm{H}$

$\mathrm{H}$

$\mathrm{H}$

$\mathrm{H}$

$\mathrm{H}$

$\mathrm{H}$

$\mathrm{H}$

$\mathrm{H}$

H

\begin{tabular}{|c|c|c|}
\hline 0.000000 & 0.000000 & 0.000000 \\
\hline 0.000000 & 0.000000 & 1.527863 \\
\hline 1.149884 & 0.000000 & 2.341123 \\
\hline 2.781337 & 0.171128 & 1.560831 \\
\hline 2.341044 & 0.825003 & 0.043938 \\
\hline 1.358424 & 0.052028 & -0.565670 \\
\hline 1.059969 & -0.137709 & 3.742246 \\
\hline-0.203239 & -0.218322 & 4.341883 \\
\hline-1.351180 & -0.189375 & 3.548672 \\
\hline-1.245386 & -0.091332 & 2.168843 \\
\hline 2.486265 & -0.246995 & 4.727996 \\
\hline-0.387075 & -0.371483 & 6.069107 \\
\hline 3.567122 & 1.211629 & 2.222611 \\
\hline 3.319285 & -1.167434 & 1.325989 \\
\hline 2.199368 & 2.291871 & -0.041586 \\
\hline 1.688178 & -0.752886 & -1.657248 \\
\hline 3.122864 & -0.716522 & -2.132971 \\
\hline-0.920238 & 1.067637 & -0.629319 \\
\hline 0.821721 & -1.440650 & -2.182094 \\
\hline-2.324466 & -0.263318 & 4.022395 \\
\hline-0.377805 & -0.977801 & -0.317624 \\
\hline 1.384032 & 2.686006 & 0.577383 \\
\hline 3.138456 & 2.746378 & 0.273444 \\
\hline 2.011961 & 2.530261 & -1.091274 \\
\hline 3.455599 & 0.304467 & -2.345141 \\
\hline 3.788774 & -1.125147 & -1.366605 \\
\hline 3.185304 & -1.322021 & -3.038538 \\
\hline-1.967565 & 0.893381 & -0.364717 \\
\hline-0.655206 & 2.080490 & -0.312730 \\
\hline-0.840641 & 1.001686 & -1.718377 \\
\hline
\end{tabular}


<smiles>CC(=O)N1C([O-])c2ccc(Cl)c(Cl)c2S(=O)(=O)N1C</smiles>

\section{$E=-2079.0648397$}

\begin{tabular}{|c|c|c|c|}
\hline C & 0.000000 & 0.000000 & 0.000000 \\
\hline C & 0.000000 & 0.000000 & 1.527863 \\
\hline C & 1.149884 & 0.000000 & 2.341123 \\
\hline$S$ & 2.781337 & 0.171128 & 1.560831 \\
\hline $\mathrm{N}$ & 2.341044 & 0.825003 & 0.043938 \\
\hline $\mathrm{N}$ & 1.358424 & 0.052028 & -0.565670 \\
\hline C & 1.059969 & -0.137709 & 3.742246 \\
\hline C & -0.203239 & -0.218322 & 4.341883 \\
\hline C & -1.351180 & -0.189375 & 3.548672 \\
\hline C & -1.245386 & -0.091332 & 2.168843 \\
\hline $\mathrm{Cl}$ & 2.486265 & -0.246995 & 4.727996 \\
\hline $\mathrm{Cl}$ & -0.387075 & -0.371483 & 6.069107 \\
\hline 0 & 3.567122 & 1.211629 & 2.222611 \\
\hline $\mathrm{O}$ & 3.319285 & -1.167434 & 1.325989 \\
\hline C & 2.199368 & 2.291871 & -0.041586 \\
\hline C & 1.688178 & -0.752886 & -1.657248 \\
\hline C & 3.122864 & -0.716522 & -2.132971 \\
\hline C & -0.920238 & 1.067637 & -0.629319 \\
\hline 0 & 0.821721 & -1.440650 & -2.182094 \\
\hline $\mathrm{H}$ & -2.324466 & -0.263318 & 4.022395 \\
\hline $\mathrm{H}$ & -2.153356 & -0.109475 & 1.575022 \\
\hline $\mathrm{H}$ & -0.377805 & -0.977801 & -0.317624 \\
\hline $\mathrm{H}$ & 1.384032 & 2.686006 & 0.577383 \\
\hline $\mathrm{H}$ & 3.138456 & 2.746378 & 0.273444 \\
\hline $\mathrm{H}$ & 2.011961 & 2.530261 & -1.091274 \\
\hline $\mathrm{H}$ & 3.455599 & 0.304467 & -2.345141 \\
\hline $\mathrm{H}$ & 3.788774 & -1.125147 & -1.366605 \\
\hline $\mathrm{H}$ & 3.185304 & -1.322021 & -3.038538 \\
\hline $\mathrm{H}$ & -0.655206 & 2.080490 & -0.312730 \\
\hline $\mathrm{H}$ & -0.840641 & 1.001686 & -1.718377 \\
\hline
\end{tabular}


<smiles>CC(=O)N1C(C)c2ccc(Cl)c(Cl)c2S(=O)(=O)N1C</smiles>

\section{$E=-2079.0986081$}

0.011018

$-0.141636$

0.097617

$-0.0819681 .608176$

1.107569

$0.017625 \quad 2.474644$

2.763196

$0.254299 \quad 1.780256$

2.393973

$0.648595 \quad 0.221796$

1.512375

$-0.285132-0.316476$

0.937355

$\begin{array}{ll}-0.023234 & 3.867759\end{array}$

$-0.348311$

$-0.113859 \quad 4.397876$

$-1.450850$

$-0.181160 \quad 3.548721$

$\begin{array}{lll}-1.266118 & -0.170395 & 2.177647\end{array}$

$2.301532 \quad 0.008880 \quad 4.932825$

$\begin{array}{lll}-0.623539 & -0.156371 & 6.116905\end{array}$

$\begin{array}{lll}3.343534 & 1.451633 & 2.381295\end{array}$

$\begin{array}{lll}3.462744 & -1.012118 \quad 1.868937\end{array}$

$2.282095 \quad 2.074968 \quad-0.112782$

1.699498

$-0.657890$

$-1.743670$

2.886898

$-0.297816-2.333210$

$-0.659371$

0.997483

$-0.588337$

0.693852

$-1.251592$

$-2.219598$

$-2.444293$

$-0.259698 \quad 3.978619$

$-2.129174$

$-0.254442$

1.522909

$-0.335346-1.078272$

$-0.233394$

1.549683

2.605264

0.508822

3.258615

2.544947

0.010094

1.996437

2.100954

$-1.166544$

3.700253

0.143692

$-1.772422$

3.059235

$-0.622229-3.352456$

$-1.739056$

0.839953

$-0.484724$

$-0.422195$

1.980813

$-0.172732$

$\mathrm{H}$

$\begin{array}{lll}-0.417619 & 0.972219 & -1.654559\end{array}$ 
<smiles>[CH2-]N1N(C(C)=O)C(C)c2ccc(Cl)c(Cl)c2S1(=O)=O</smiles>

\section{$E=-2079.076127$}

C

C

\section{C}

\section{S}

$$
\text { N }
$$

N

$$
\text { C }
$$

c

\section{c}$$
\mathrm{H}
$$

\subsection{3}

0.009428

1.150076

2.780444

2.349430

1.403124

1.033784

$-0.232035$

$-1.370631$

$-1.244403$

2.437840

$-0.436440$

3.590699

3.283500

2.106492

1.671945

3.076388

$-0.823335$

0.809948

$-2.348403$

$-2.142153$

$-0.365086$

1.411571

3.071998

3.288340

3.797731

3.148777

$-1.876758$

$-0.454562$

$-0.745306$
$-0.093208$

$-0.029388$

1.558364

$-0.038635 \quad 2.374901$

$0.083595 \quad 1.563460$

$0.781347 \quad 0.062498$

$-0.103552-0.473525$

$-0.124368 \quad 3.768999$

$-0.147300 \quad 4.355333$

$-0.101940 \quad 3.557459$

$-0.052430 \quad 2.179285$

$-0.250886 \quad 4.774991$

$-0.248085 \quad 6.083512$

$1.047087 \quad 2.292218$

$-1.282924 \quad 1.396665$

$2.201593 \quad-0.078672$

$-0.717421-1.665428$

$-0.543512-2.186793$

$0.978600 \quad-0.646862$

$-1.365178 \quad-2.254022$

$-0.124623 \quad 4.027697$

$-0.052385 \quad 1.568614$

$-1.075312 \quad-0.236165$

$2.562721 \quad 0.704873$

$2.699875 \quad 0.048715$

$0.522804-2.312081$

$-0.940911-1.467192$

$-1.072504 \quad-3.138067$

$0.880237-0.362131$

$1.976804-0.410120$

$0.831134-1.727432$ 


$\begin{array}{lrrr}\mathrm{H}_{2} \mathrm{O} & & & \\ \mathrm{E}=-75.7523279 & & \\ \mathrm{O} & 0.002039 & 0.000000 & 0.001553 \\ \mathrm{H} & -0.000718 & -0.000000 & 0.967620 \\ \mathrm{H} & 0.932611 & -0.000000 & -0.257926 \\ & & & \\ & & & \\ \mathrm{OH}^{-} & & & \\ \mathrm{E}=-75.7523279 & & \\ \mathrm{O} & 0.000000 & 0.000000 & 0.001647 \\ \mathrm{H} & 0.000000 & 0.000000 & 0.973227 \\ & & & \\ \mathrm{MeOH} & & & \\ \mathrm{E}=-115.6609312 & & \\ \mathrm{C} & 0.000070 & -0.000000 & 0.002523 \\ \mathrm{O} & 0.001313 & 0.000000 & 1.422005 \\ \mathrm{H} & 0.906690 & -0.000000 & 1.751224 \\ \mathrm{H} & -0.523712 & -0.890210 & -0.358823 \\ \mathrm{H} & -0.523712 & 0.890210 & -0.358823 \\ \mathrm{H} & 1.013002 & -0.000000 & -0.413393\end{array}$

\begin{tabular}{|c|c|c|c|}
\hline \multicolumn{4}{|c|}{$\begin{array}{l}\mathrm{MeO}^{-} \\
E=-115.0458769\end{array}$} \\
\hline c & -0.000043 & 0.000139 & -0.002304 \\
\hline 0 & 0.000023 & -0.000141 & 1.334566 \\
\hline H & 1.025462 & -0.000020 & -0.491240 \\
\hline H & -0.513063 & -0.887773 & -0.491136 \\
\hline$H$ & -0.512768 & 0.888438 & -0.490941 \\
\hline
\end{tabular}

\section{$t-\mathrm{BuOH}$}

$\begin{array}{cccc}E=-233.560657 & & \\ \text { C } & -0.003353 & -0.000001 & 0.007977 \\ C & 0.002976 & -0.000001 & 1.531408 \\ \text { O } & 1.386275 & -0.000001 & 1.894183 \\ C & -0.672060 & -1.258353 & 2.077316 \\ C & -0.672059 & 1.258353 & 2.077315\end{array}$




$\begin{array}{llll}\mathrm{H} & -1.029051 & -0.000001 & -0.374258 \\ \mathrm{H} & 0.514134 & -0.887355 & -0.369439 \\ \mathrm{H} & -1.723062 & -1.306180 & 1.772269 \\ \mathrm{H} & 0.514135 & 0.887352 & -0.369440 \\ \mathrm{H} & -0.640916 & -1.271011 & 3.174206 \\ \mathrm{H} & -0.157988 & -2.151099 & 1.707949 \\ \mathrm{H} & -0.640915 & 1.271012 & 3.174205 \\ \mathrm{H} & -0.157985 & 2.151098 & 1.707947 \\ \mathrm{H} & -1.723060 & 1.306181 & 1.772268 \\ \mathrm{H} & 1.453945 & -0.000001 & 2.860597\end{array}$

\section{$t-\mathrm{BuO}^{-}$}

\section{$E=-232.9543475$}

$\begin{array}{llll}\text { C } & -0.004841 & -0.000215 & 0.010064\end{array}$

$\begin{array}{llll}C & -0.004309 & 0.000163 & 1.570567\end{array}$

$\begin{array}{llll}0 & 1.240000 & 0.000475 & 2.069534\end{array}$

$\begin{array}{llll}\text { C } & -0.813246 & -1.253841 & 2.026479\end{array}$

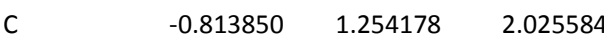

$\begin{array}{llll}\text { H } & -1.012918 & 0.000134 & -0.438166\end{array}$

$\begin{array}{llll}\text { H } & 0.539057 & -0.887201 & -0.340144\end{array}$

$\begin{array}{llll}\text { H } & -1.844463 & -1.288405 & 1.636007\end{array}$

$\begin{array}{llll}\text { H } & 0.539715 & 0.886314 & -0.340341\end{array}$

$\begin{array}{llll}H & -0.848187 & -1.267403 & 3.123649\end{array}$

$\begin{array}{llll}\text { H } & -0.278327 & -2.154131 & 1.697019\end{array}$

$\begin{array}{llll}\text { H } & -0.849835 & 1.268299 & 3.122741\end{array}$

$\begin{array}{llll}\text { H } & -0.278951 & 2.154584 & 1.696361\end{array}$

$\begin{array}{llll}\text { H } & -1.844713 & 1.288242 & 1.634155\end{array}$

\section{$\mathrm{AcOH}$}

\section{$E=-228.9820965$}

$\begin{array}{llll}\text { C } & 0.001312 & 0.000000 & 0.001645 \\ \text { C } & 0.002190 & 0.000000 & 1.517701 \\ \text { O } & 1.210085 & -0.000000 & 2.130911 \\ \text { O } & -1.001584 & 0.000000 & 2.173663 \\ \text { H } & 0.998068 & -0.000000 & -0.445678 \\ \text { H } & -0.544271 & -0.881952 & -0.341892 \\ \text { H } & -0.544271 & 0.881952 & -0.341892 \\ \text { H } & 1.931393 & -0.000000 & 1.486280\end{array}$




$\mathrm{AcO}^{-}$
$\begin{array}{llll}\mathrm{E}=-228.4341997 & & \\ \mathrm{C} & 0.000674 & -0.000000 & 0.001819 \\ \mathrm{C} & 0.000438 & 0.000000 & 1.559417 \\ \mathrm{O} & 1.124940 & 0.000000 & 2.115411 \\ \mathrm{O} & -1.139083 & -0.000000 & 2.086382 \\ \mathrm{H} & 1.017902 & 0.000000 & -0.401840 \\ \mathrm{H} & -0.539935 & -0.882032 & -0.362440 \\ \mathrm{H} & -0.539935 & 0.882032 & -0.362440\end{array}$


trans-3b and cis-3b were calculated at B3LYP[7]/6-31G (pcm ${ }^{[8,9]: ~ D M S O) ~}$<smiles>CC(=O)NC1c2ccc(Cl)c(Cl)c2S(=O)(=O)N(C)[C@@H]1C</smiles>

trans-3b

$E=-2118.9937322$

C

C

C

$\mathrm{O}$

C

C

C

$\mathrm{H}$

H

$\mathrm{H}$

$\mathrm{H}$

\begin{tabular}{|c|c|c|}
\hline 0.037409 & 0.183665 & -0.034060 \\
\hline 0.042526 & 0.068245 & 1.368455 \\
\hline 1.300529 & -0.009517 & 1.989493 \\
\hline 2.497230 & 0.093328 & 1.270276 \\
\hline 2.446429 & 0.210038 & -0.116728 \\
\hline 1.215716 & 0.244415 & -0.771027 \\
\hline 1.391194 & -0.213322 & 3.870205 \\
\hline-0.332555 & -0.586533 & 4.322782 \\
\hline-1.253517 & 0.342849 & 3.607924 \\
\hline-1.315018 & 0.014176 & 2.090277 \\
\hline-2.280750 & 0.878727 & 1.413367 \\
\hline-3.472652 & 0.403459 & 0.925809 \\
\hline-3.776954 & -0.814854 & 0.995123 \\
\hline 2.284808 & -1.517814 & 4.290996 \\
\hline 1.774774 & 1.218641 & 4.564386 \\
\hline-0.638691 & -2.032967 & 4.393522 \\
\hline 4.095437 & 0.092751 & 2.114758 \\
\hline 3.953676 & 0.323451 & -1.113312 \\
\hline-4.399245 & 1.433740 & 0.324352 \\
\hline 1.182871 & 0.328822 & -1.849549 \\
\hline-0.912275 & 0.238370 & -0.549216 \\
\hline-1.722787 & -0.998627 & 1.975532 \\
\hline-0.844538 & -2.498445 & 3.422201 \\
\hline 0.206089 & -2.539327 & 4.859714 \\
\hline-1.509772 & -2.144225 & 5.042683 \\
\hline-4.833361 & 1.033173 & -0.594946 \\
\hline-5.222398 & 1.630508 & 1.020787 \\
\hline-3.899374 & 2.379683 & 0.103576 \\
\hline-2.629309 & 0.296567 & 4.285088 \\
\hline-0.834581 & 1.347059 & 3.747570 \\
\hline-2.080852 & 1.868564 & 1.352938 \\
\hline-2.508839 & 0.409942 & 5.36566 \\
\hline
\end{tabular}




$\begin{array}{llll}H & -3.250097 & 1.118008 & 3.919635 \\ H & -3.152068 & -0.642644 & 4.081462\end{array}$<smiles>CC(=O)NC1c2ccc(Cl)c(Cl)c2S(=O)(=O)N(C)C1C</smiles>

\section{cis-3b}

\section{$E=-2118.9920237$}

$0.103790 \quad 0.478742 \quad-0.053957$

C

C

$0.086540 \quad 0.185029 \quad 1.320200$

$1.326881-0.066854 \quad 1.931538$

$\begin{array}{llll}\text { C } & 2.533060 & -0.011725 & 1.223691\end{array}$

$\begin{array}{llll}\text { C } & 2.505390 & 0.283555 & -0.138537\end{array}$

$\begin{array}{llll}\text { C } & 1.291771 & 0.526848 & -0.778239\end{array}$

S $\quad 1.399437 \quad-0.469045 \quad 3.785364$

$\begin{array}{llll}\mathrm{N} & -0.348551 & -0.454743 & 4.326774\end{array}$

$\begin{array}{llll}\text { C } & -1.192090 & 0.501689 & 3.552529\end{array}$

$\begin{array}{llll}\text { C } & -1.257313 & 0.098658 & 2.051431\end{array}$

$\begin{array}{llll}\text { N } & -2.303101 & 0.848063 & 1.362730\end{array}$

$\begin{array}{llll}\text { C } & -3.464099 & 0.249308 & 0.936094\end{array}$

$\begin{array}{llll}\text { O } & -3.660156 & -0.984679 & 1.073037\end{array}$

$\begin{array}{llll}\text { O } & 1.966886 & -1.987889 & 4.023674\end{array}$

$\begin{array}{llll}\text { O } & 2.175923 & 0.733890 & 4.576685\end{array}$

$\begin{array}{llll}\text { C } & -0.896321 & -1.805914 & 4.590414\end{array}$

$\mathrm{Cl} \quad 4.114066 \quad-0.326740 \quad 2.040011$

Cl $\quad 4.024563 \quad 0.356546 \quad-1.121374$

$\begin{array}{llll}\text { C } & -4.491372 & 1.167194 & 0.316628\end{array}$

$\begin{array}{llll}\text { H } & 1.278089 & 0.752773 & -1.836646\end{array}$

$\begin{array}{llll}\text { H } & -0.833525 & 0.672862 & -0.557711\end{array}$

$\begin{array}{llll}H & -1.598795 & -0.943829 & 2.005406\end{array}$

$\begin{array}{llll}\text { H } & -1.209235 & -2.347942 & 3.689752\end{array}$

$\begin{array}{llll}H & -0.149307 & -2.397208 & 5.117605\end{array}$

$\begin{array}{llll}\text { H } & -1.759417 & -1.661402 & 5.245793\end{array}$

$\begin{array}{llll}\text { H } & -4.927500 & 0.678796 & -0.558034\end{array}$

$\begin{array}{llll}\text { H } & -5.300021 & 1.344628 & 1.034918\end{array}$

$\begin{array}{llll}\text { H } & -4.075448 & 2.132740 & 0.018806\end{array}$

$\begin{array}{llll}\text { H } & -2.196992 & 0.343920 & 3.960100\end{array}$ 


$\begin{array}{llll}\mathrm{C} & -0.793180 & 1.959037 & 3.812071 \\ \mathrm{H} & -2.197973 & 1.847348 & 1.252383 \\ \mathrm{H} & -0.761683 & 2.140877 & 4.888955 \\ \mathrm{H} & 0.185295 & 2.215026 & 3.396852 \\ \mathrm{H} & -1.537009 & 2.632662 & 3.375815\end{array}$

50

$\begin{array}{lrrr}\text { E }=-1160.584351 & \\ \text { C } & -0.051277 & -0.066852 & -0.092053 \\ \text { C } & -0.118922 & -0.129319 & 1.302261 \\ \text { C } & 1.037260 & -0.101805 & 2.079688 \\ \text { C } & 2.288209 & -0.027542 & 1.470422 \\ \text { C } & 2.381284 & 0.007790 & 0.081245 \\ \text { C } & 1.217462 & -0.013805 & -0.684728 \\ \text { S } & 1.410284 & 0.008462 & -2.465117 \\ \text { O } & 2.806870 & -0.331811 & -2.736441 \\ \text { C } & -1.331136 & -0.075248 & -0.870933 \\ \text { N } & -1.942137 & -1.346406 & -0.951703 \\ \text { C } & -3.258519 & -1.704950 & -0.813840 \\ \text { C } & -4.259704 & -0.661099 & -0.385163 \\ \text { N } & 0.431183 & -1.246609 & -2.946723 \\ \text { C } & -0.507527 & -1.010841 & -4.051117 \\ \text { O } & 0.860633 & 1.244842 & -3.011707 \\ \text { C } & -1.846045 & 1.036087 & -1.399390 \\ \text { O } & -3.591528 & -2.868170 & -1.016935 \\ \text { H } & -1.095433 & -0.193074 & 1.776607 \\ \text { H } & 0.958394 & -0.140606 & 3.162301 \\ \text { H } & 3.192690 & -0.009253 & 2.070750 \\ \text { H } & 3.347916 & 0.038055 & -0.411278 \\ \text { H } & 0.995451 & -2.088378 & -3.065321 \\ \text { H } & -1.112292 & -1.915091 & -4.149410 \\ \text { H } & -1.158721 & -0.174921 & -3.792369 \\ \text { H } & -0.006292 & -0.802245 & -5.001990 \\ \text { H } & -2.766108 & 1.023910 & -1.975986 \\ \text { H } & -1.341886 & 1.987038 & -1.264318 \\ \text { H } & -1.317883 & -2.123805 & -1.156674 \\ \text { H } & -3.823800 & 0.106064 & 0.258394 \\ \text { H } & -4.684409 & -0.170124 & -1.266935 \\ \text { H } & -5.067127 & -1.176837 & 0.137789\end{array}$

Io+

\section{$E=-1161.014911$}

$\begin{array}{llll}\text { C } & 0.010679 & 0.098132 & 0.027007\end{array}$

$\begin{array}{llll}\text { C } & 0.031700 & 0.053905 & 1.420959\end{array}$

C $1.249183 \quad 0.004412 \quad 2.101188$ 


$\begin{array}{llll}\text { C } & 2.442944 & -0.063134 & 1.360970 \\ \text { C } & 2.420505 & -0.059112 & -0.026242 \\ \text { C } & 1.198691 & 0.045363 & -0.693943 \\ \text { C } & 1.198227 & 0.031823 & 3.582025 \\ \text { C } & 0.401868 & -0.990723 & 4.302508 \\ \text { S } & 4.029612 & -0.251324 & 2.170607 \\ \text { O } & 4.345248 & 0.971100 & 2.915532 \\ \text { N } & 3.637371 & -1.385274 & 3.316197 \\ \text { C } & 3.513473 & -2.774760 & 2.849015 \\ \text { O } & 4.973867 & -0.736746 & 1.174889 \\ \text { N } & 1.826491 & 1.007685 & 4.179922 \\ \text { C } & 2.002954 & 1.208743 & 5.614843 \\ \text { O } & 1.468217 & 0.470001 & 6.392319 \\ \text { C } & 2.894615 & 2.365715 & 5.917123 \\ \text { H } & 3.350608 & -0.154687 & -0.578106 \\ \text { H } & 1.182237 & 0.060082 & -1.779099 \\ \text { H } & -0.941553 & 0.159455 & -0.490216 \\ \text { H } & -0.897929 & 0.079710 & 1.983108 \\ \text { H } & 2.342884 & 1.665713 & 3.589203 \\ \text { H } & 2.518491 & 3.269027 & 5.424678 \\ \text { H } & 2.939456 & 2.513310 & 6.995709 \\ \text { H } & 3.898903 & 2.162715 & 5.526435 \\ \text { H } & 4.260572 & -1.278496 & 4.120704 \\ \text { H } & 3.173758 & -3.370980 & 3.697587 \\ \text { H } & 2.754681 & -2.825886 & 2.062662 \\ \text { H } & 4.458327 & -3.174427 & 2.470230 \\ \text { H } & -0.057803 & -1.674727 & 3.590233 \\ \text { H } & 1.064408 & -1.550247 & 4.973670 \\ \text { H } & -0.358453 & -0.517271 & 4.931998\end{array}$

\section{TS between $\mathrm{Io}^{+}$and $\mathrm{Ilo}^{+}$}

\section{$E=-1161.011215$}

$\begin{array}{lccc}\text { nimag }=1 & & \\ \text { C } & -2.816925 & 0.382303 & -0.685777 \\ \text { C } & -3.246034 & 1.700852 & -0.544564 \\ \text { C } & -2.381099 & 2.675778 & -0.046256 \\ \text { C } & -1.074550 & 2.353524 & 0.324121 \\ \text { C } & -0.632124 & 1.041595 & 0.178249 \\ \text { C } & -1.510290 & 0.084284 & -0.327478 \\ \text { C } & 0.767949 & 0.655338 & 0.561636 \\ \text { C } & 1.208711 & 0.958236 & 1.959481 \\ \text { S } & -0.840252 & -1.547300 & -0.466605 \\ \text { N } & 0.262853 & -1.370936 & 0.833635 \\ \text { C } & -0.289513 & -1.714929 & 2.159838 \\ \text { N } & 1.629838 & 0.654406 & -0.466797 \\ \text { C } & 3.044721 & 0.459216 & -0.419620 \\ \text { O } & 3.608507 & 0.273670 & 0.629651 \\ \text { C } & 3.688551 & 0.516419 & -1.770128 \\ \text { O } & -1.862797 & -2.536399 & -0.181122 \\ \text { O } & -0.000143 & -1.668666 & -1.650872\end{array}$


$\begin{array}{cccc}\text { H } & -3.484557 & -0.394208 & -1.048325 \\ \text { H } & -4.264879 & 1.964334 & -0.812895 \\ \text { H } & -2.729514 & 3.697387 & 0.067869 \\ \text { H } & -0.407321 & 3.112063 & 0.725773 \\ \text { H } & 1.230415 & 0.702903 & -1.403322 \\ \text { H } & 4.755796 & 0.327879 & -1.665248 \\ \text { H } & 3.239864 & -0.231530 & -2.434203 \\ \text { H } & 3.527769 & 1.504086 & -2.216423 \\ \text { H } & 1.115303 & -1.905580 & 0.619060 \\ \text { H } & 0.501544 & -1.568535 & 2.896736 \\ \text { H } & -1.127661 & -1.051907 & 2.384135 \\ \text { H } & -0.622622 & -2.754928 & 2.182327 \\ \text { H } & 0.342613 & 0.966330 & 2.622184 \\ \text { H } & 1.966466 & 0.264694 & 2.319181 \\ \text { H } & 1.654339 & 1.962630 & 1.947993\end{array}$

\section{Ilo+}

\section{$E=-1161.029160$}

$\begin{array}{llll}\text { C } & -0.008063 & 0.024232 & 0.010188\end{array}$

$\begin{array}{llll}\text { C } & -0.002421 & -0.017532 & 1.398685\end{array}$

C $1.219863 \quad-0.0142292 .061843$

C $2.450335 \quad 0.022196 \quad 1.420152$

C $2.427924 \quad 0.067368 \quad 0.027124$

$\begin{array}{llll}\text { C } & 1.214527 & 0.067829 & -0.664437\end{array}$

S $\quad \begin{array}{llll}1.015552 & -0.070627 & 3.809785\end{array}$

$\begin{array}{llll}\text { N } & -0.733680 & -0.608608 & 3.622892\end{array}$

$\begin{array}{llll}\text { C } & -1.230201 & 0.007076 & 2.296517\end{array}$

$\begin{array}{llll}\text { C } & -0.865588 & -2.090329 & 3.750316\end{array}$

$\begin{array}{llll}\text { N } & -1.550708 & 1.396696 & 2.576306\end{array}$

$\begin{array}{llll}\text { C } & -2.335163 & 1.800618 & 3.615976\end{array}$

C $-2.614142 \quad 3.270975 \quad 3.714479$

C $\quad-2.427833 \quad-0.754792 \quad 1.752302$

$\begin{array}{llll}\text { O } & 1.737852 & -1.159061 & 4.425635\end{array}$

$\begin{array}{llll}0 & 0.948488 & 1.231690 & 4.434098\end{array}$

$\begin{array}{llll}\mathrm{O} & -2.743504 & 0.986413 & 4.448107\end{array}$

$\begin{array}{llll}\text { H } & 3.384397 & 0.010254 & 1.974094\end{array}$

H $3.3641010 .094789-0.520915$

H $\quad 1.218860 \quad 0.098510 \quad-1.749648$

$\begin{array}{llll}H & -0.941977 & 0.021541 & -0.543866\end{array}$

$\begin{array}{llll}H & -1.154390 & 2.098310 & 1.958173\end{array}$

$\begin{array}{llll}H & -2.339748 & 3.815680 & 2.809628\end{array}$

$\begin{array}{llll}H & -3.677715 & 3.409508 & 3.923203\end{array}$

$\begin{array}{llll}H & -2.047928 & 3.671359 & 4.561938\end{array}$

$\begin{array}{llll}\text { H } & -1.306224 & -0.161364 & 4.372934\end{array}$

$\begin{array}{llll}H & -1.927964 & -2.333131 & 3.741389\end{array}$

H $\quad-0.345019 \quad-2.578983 \quad 2.925419$

$\begin{array}{llll}\text { H } & -0.429712 & -2.382612 & 4.704849\end{array}$

$\begin{array}{llll}\text { H } & -2.160423 & -1.770619 & 1.454736\end{array}$

$\begin{array}{llll}\text { H } & -3.234562 & -0.778156 & 2.489257\end{array}$

H $\quad-2.782742 \quad-0.212850 \quad 0.872101$ 
20

$E=-1160.614135$

$\begin{array}{llll}\text { C } & 0.005699 & 0.001276 & -0.003233\end{array}$

$\begin{array}{llll}\text { C } & 0.003814 & -0.000907 & 1.385808\end{array}$

$\begin{array}{llll}\text { C } & 1.157059 & 0.001269 & 2.151696\end{array}$

$\begin{array}{llll}\text { C } & 2.394115 & -0.000726 & 1.513591\end{array}$

$\begin{array}{llll}\text { C } & 2.427616 & 0.004192 & 0.119267\end{array}$

$\begin{array}{llll}\text { C } & 1.247672 & 0.008700 & -0.633776\end{array}$

$\begin{array}{llll}\mathrm{S} & -1.411402 & -0.077177 & 2.440491\end{array}$

$\begin{array}{llll}\mathrm{N} & -0.518006 & 0.327452 & 3.803163\end{array}$

C $0.921159 \quad-0.014136 \quad 3.657624$

N $\quad 1.232634 \quad-1.365673 \quad 4.119780$

C $1.463724-1.769426 \quad 5.407815$

$\begin{array}{llll}\text { O } & 1.542253 & -1.000172 & 6.356805\end{array}$

C $1.754651 \quad 1.063249 \quad 4.350550$

$\begin{array}{llll}\mathrm{O} & -2.385278 & 0.973523 & 2.156826\end{array}$

$\begin{array}{llll}\text { O } & -1.928754 & -1.447359 & 2.513829\end{array}$

C $\quad-1.178827 \quad-0.006425 \quad 5.064920$

$\begin{array}{llll}\text { C } & 1.588911 & -3.267359 & 5.575775\end{array}$

$\begin{array}{llll}\text { H } & -0.920123 & 0.001966 & -0.571399\end{array}$

H $1.298806 \quad 0.018656 \quad-1.718212$

$\begin{array}{llll}\text { H } & 3.386100 & 0.007893 & -0.391334\end{array}$

$\begin{array}{llll}H & 3.314633 & -0.003236 & 2.090958\end{array}$

$\begin{array}{llll}\text { H } & 1.093177 & -2.106051 & 3.439802\end{array}$

H $\quad 1.972062 \quad-3.763455 \quad 4.680490$

H $2.243634 \quad-3.475906 \quad 6.423660$

H $\quad 0.595982-3.674389 \quad 5.799088$

$\begin{array}{llll}\text { H } & -1.212610 & -1.088354 & 5.240773\end{array}$

$\begin{array}{llll}H & -0.634948 & 0.476101 & 5.878306\end{array}$

$\begin{array}{llll}\text { H } & -2.197719 & 0.389729 & 5.043941\end{array}$

H $1.461722 \quad 1.183941 \quad 5.392451$

$\begin{array}{llll}H & 2.813485 & 0.789559 & 4.321608\end{array}$

H $\quad 1.610355 \quad 2.006167 \quad 3.817072$

conformer of $\mathbf{5 a}$, structure was obtained from the scan calculations
$E=-2079.704733$
C $\quad-0.008283 \quad-0.000614-0.064469$
C $\quad-0.039985 \quad-0.209636 \quad 1.321363$
C $1.164069-0.307343 \quad 2.021858$
C $2.378715 \quad-0.128611 \quad 1.380703$
C $2.399965 \quad 0.1905290 .026723$
$\begin{array}{llll}\text { C } & 1.212161 & 0.269384 & -0.708161\end{array}$
$\begin{array}{llll}\text { C } & -1.297843 & -0.223888 & 2.132283\end{array}$
$\begin{array}{llll}\text { C } & -1.989006 & 0.902010 & 2.327096\end{array}$
$\begin{array}{llll}\text { S } & -1.539764 & -0.135347 & -1.030967\end{array}$
$\begin{array}{lllll}\text { O } & -2.238889 & 1.142291 & -0.987662\end{array}$
$\begin{array}{lllll}\mathrm{O} & -1.187614 & -0.740083 & -2.309769\end{array}$
$\begin{array}{llll}\mathrm{N} & -2.408591 & -1.256685 & -0.175876\end{array}$ 


$\begin{array}{cccc}\text { C } & -3.796309 & -0.943142 & 0.188466 \\ \text { N } & -1.606285 & -1.463913 & 2.722864 \\ \text { C } & -2.064182 & -1.755513 & 3.985383 \\ \text { O } & -2.372591 & -2.910045 & 4.257889 \\ \text { C } & -2.141608 & -0.651614 & 5.009789 \\ \text { H } & 1.138216 & -0.497316 & 3.091823 \\ \text { H } & 3.312007 & -0.196103 & 1.930600 \\ \text { Cl } & 3.937683 & 0.495050 & -0.719642 \\ \text { Cl } & 1.291197 & 0.772324 & -2.362696 \\ \text { H } & -2.305165 & -2.166170 & -0.626579 \\ \text { H } & -4.137360 & -1.739422 & 0.854287 \\ \text { H } & -3.821937 & 0.004007 & 0.727297 \\ \text { H } & -4.456676 & -0.885744 & -0.682436 \\ \text { H } & -2.895550 & 0.919494 & 2.923795 \\ \text { H } & -1.648901 & 1.833938 & 1.887693 \\ \text { H } & -1.499173 & -2.279987 & 2.124698 \\ \text { H } & -1.377712 & 0.115348 & 4.864383 \\ \text { H } & -3.124352 & -0.170802 & 4.964070 \\ \text { H } & -2.032459 & -1.109273 & 5.994565\end{array}$

\begin{tabular}{|c|c|c|c|}
\hline \multicolumn{4}{|c|}{$E=-2080.132097$} \\
\hline C & 0.031841 & 0.259228 & 0.116888 \\
\hline$C$ & 0.149683 & 0.102638 & 1.491514 \\
\hline C & 1.408268 & -0.073418 & 2.061423 \\
\hline C & 2.545880 & -0.135506 & 1.247772 \\
\hline 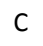 & 2.439376 & 0.057655 & -0.132545 \\
\hline C & 1.165007 & 0.251607 & -0.688183 \\
\hline C & 1.467953 & -0.126872 & 3.555284 \\
\hline C & 0.741571 & -1.211505 & 4.264896 \\
\hline S & 4.116049 & -0.477795 & 2.069716 \\
\hline 0 & 4.596139 & 0.764248 & 2.665189 \\
\hline IV & 3.513141 & -1.398786 & 3.326892 \\
\hline C & 3.297848 & -2.825789 & 3.035113 \\
\hline 0 & 4.970462 & -1.267744 & 1.205880 \\
\hline$N$ & 1.924539 & 0.955881 & 4.142309 \\
\hline C & 2.024968 & 1.220670 & 5.570689 \\
\hline 0 & 1.618594 & 0.415719 & 6.361170 \\
\hline C & 2.657699 & 2.540338 & 5.862864 \\
\hline $\mathrm{Cl}$ & 3.827635 & 0.097293 & -1.152775 \\
\hline $\mathrm{Cl}$ & 0.969526 & 0.479174 & -2.390245 \\
\hline $\mathrm{H}$ & -0.944774 & 0.399996 & -0.334714 \\
\hline H & -0.737058 & 0.134071 & 2.117468 \\
\hline $\mathrm{H}$ & 2.311819 & 1.686715 & 3.540714 \\
\hline $\mathrm{H}$ & 2.087188 & 3.340948 & 5.379106 \\
\hline $\mathrm{H}$ & 2.678734 & 2.696219 & 6.940970 \\
\hline $\mathrm{H}$ & 3.677055 & 2.560187 & 5.460507 \\
\hline $\mathrm{H}$ & 4.076433 & -1.233259 & 4.167395 \\
\hline $\mathrm{H}$ & 2.849603 & -3.273613 & 3.923406 \\
\hline $\mathrm{H}$ & 2.599708 & -2.918374 & 2.198053 \\
\hline
\end{tabular}




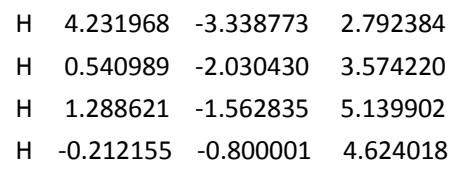

$\mathrm{Ila}^{+}$

$E=-2080.152661$

$\begin{array}{llll}\text { C } & -0.005795 & 0.013314 & 0.007742\end{array}$

$\begin{array}{llll}\text { C } & 0.002871 & 0.001152 & 1.395480\end{array}$

$\begin{array}{llll}\text { C } & 1.219661 & -0.010084 & 2.062997\end{array}$

$\begin{array}{llll}\text { C } & 2.448736 & 0.004643 & 1.413602\end{array}$

$\begin{array}{llll}\text { C } & 2.424503 & 0.012680 & 0.014502\end{array}$

$\begin{array}{llll}\text { C } & 1.209238 & 0.015047 & -0.672460\end{array}$

S $\quad 1.011216 \quad-0.058183 \quad 3.819239$

$\begin{array}{llll}\text { N } & -0.746641 & -0.549631 & 3.617494\end{array}$

$\begin{array}{llll}\text { C } & -1.223111 & 0.072614 & 2.291941\end{array}$

$\begin{array}{llll}\text { N } & -1.496925 & 1.473321 & 2.565458\end{array}$

C $\quad-2.268675 \quad 1.905732 \quad 3.604511$

C $-2.532257 \quad 3.379848 \quad 3.675466$

$\begin{array}{llll}\text { C } & -2.443243 & -0.653050 & 1.748133\end{array}$

$\begin{array}{llll}\text { O } & 1.686838 & -1.174034 & 4.432290\end{array}$

$\begin{array}{llll}\mathrm{O} & 0.990426 & 1.251127 & 4.426121\end{array}$

$\begin{array}{llll}\text { C } & -0.916370 & -2.029336 & 3.738734\end{array}$

$\begin{array}{llll}\text { Cl } & 3.915383 & 0.021101 & 2.311375\end{array}$

$\begin{array}{llll}\text { Cl } & 3.900229 & 0.020550 & -0.881021\end{array}$

$\begin{array}{llll}\text { O } & -2.676753 & 1.112299 & 4.456928\end{array}$

$\begin{array}{llll}\text { H } & 1.221224 & 0.017663 & -1.757791\end{array}$

$\begin{array}{llll}H & -0.938042 & 0.021147 & -0.548393\end{array}$

$\begin{array}{llll}\text { H } & -1.117525 & 2.157966 & 1.917935\end{array}$

$\begin{array}{llll}H & -2.283974 & 3.898776 & 2.747823\end{array}$

$\begin{array}{llll}H & -3.586922 & 3.532813 & 3.916453\end{array}$

$\begin{array}{llll}H & -1.934953 & 3.796246 & 4.493289\end{array}$

$\begin{array}{llll}\text { H } & -1.316205 & -0.085607 & 4.364319\end{array}$

$\begin{array}{llll}\text { H } & -1.986382 & -2.236428 & 3.719645\end{array}$

$\begin{array}{llll}H & -0.405679 & -2.525084 & 2.912058\end{array}$

$\begin{array}{llll}\text { H } & -0.497316 & -2.336816 & 4.695698\end{array}$

$\begin{array}{llll}\text { H } & -2.210562 & -1.678848 & 1.456376\end{array}$

$\begin{array}{llll}\text { H } & -3.251660 & -0.644274 & 2.483932\end{array}$

$\begin{array}{llll}\text { H } & -2.779538 & -0.103828 & 0.865090\end{array}$

\section{isomer of $\mathbf{2 a}$}

\section{$E=-2079.743264$}

C $0.001775 \quad-0.005684 \quad-0.001929$

$\begin{array}{llll}\text { C } & 0.001069 & -0.002371 & 1.387897\end{array}$

C $1.161286-0.002132 \quad 2.140209$

$\begin{array}{llll}\text { C } & 2.394848 & 0.006235 & 1.497098\end{array}$

C $2.426393 \quad 0.022373 \quad 0.107238$

C $1.244350 \quad 0.017165 \quad-0.639256$ 


$\begin{array}{lccc}\text { S } & -1.414219 & -0.094020 & 2.456881 \\ \text { N } & -0.500214 & 0.290809 & 3.806962 \\ \text { C } & 0.934506 & -0.054637 & 3.646795 \\ \text { N } & 1.238427 & -1.419756 & 4.066708 \\ \text { C } & 1.486965 & -1.858566 & 5.342448 \\ \text { O } & 1.605081 & -1.112472 & 6.304418 \\ \text { C } & 1.777775 & 1.001414 & 4.360716 \\ \text { O } & -2.384633 & 0.963900 & 2.209182 \\ \text { O } & -1.917056 & -1.467812 & 2.498146 \\ \text { C } & -1.151387 & -0.048304 & 5.073745 \\ \text { C } & 1.345170 & 0.031366 & -2.369587 \\ \text { C } & -1.482617 & -0.045299 & -0.879332 \\ \text { C } & 1.581316 & -3.362260 & 5.471028 \\ \text { H } & 3.377046 & 0.038305 & -0.416632 \\ \text { H } & 3.321330 & 0.000550 & 2.063267 \\ \text { H } & 1.056065 & -2.142523 & 3.377191 \\ \text { H } & 1.957778 & -3.840008 & 4.562907 \\ \text { H } & 2.229105 & -3.606569 & 6.314434 \\ \text { H } & 0.579777 & -3.756322 & 5.678872 \\ \text { H } & -1.177169 & -1.130200 & 5.249670 \\ \text { H } & -0.606880 & 0.440423 & 5.882601 \\ \text { H } & -2.172620 & 0.341344 & 5.059602 \\ \text { H } & 1.485611 & 1.103014 & 5.404559 \\ \text { H } & 2.834058 & 0.719248 & 4.328289 \\ \text { H } & 1.640591 & 1.955922 & 3.846276\end{array}$




\section{Single crystal X-ray diffraction data}

1a (CCDC 1995299)

The crystal was grown from ethyl acetate by allowing a solution of the compound in a vial to evaporate slowly.

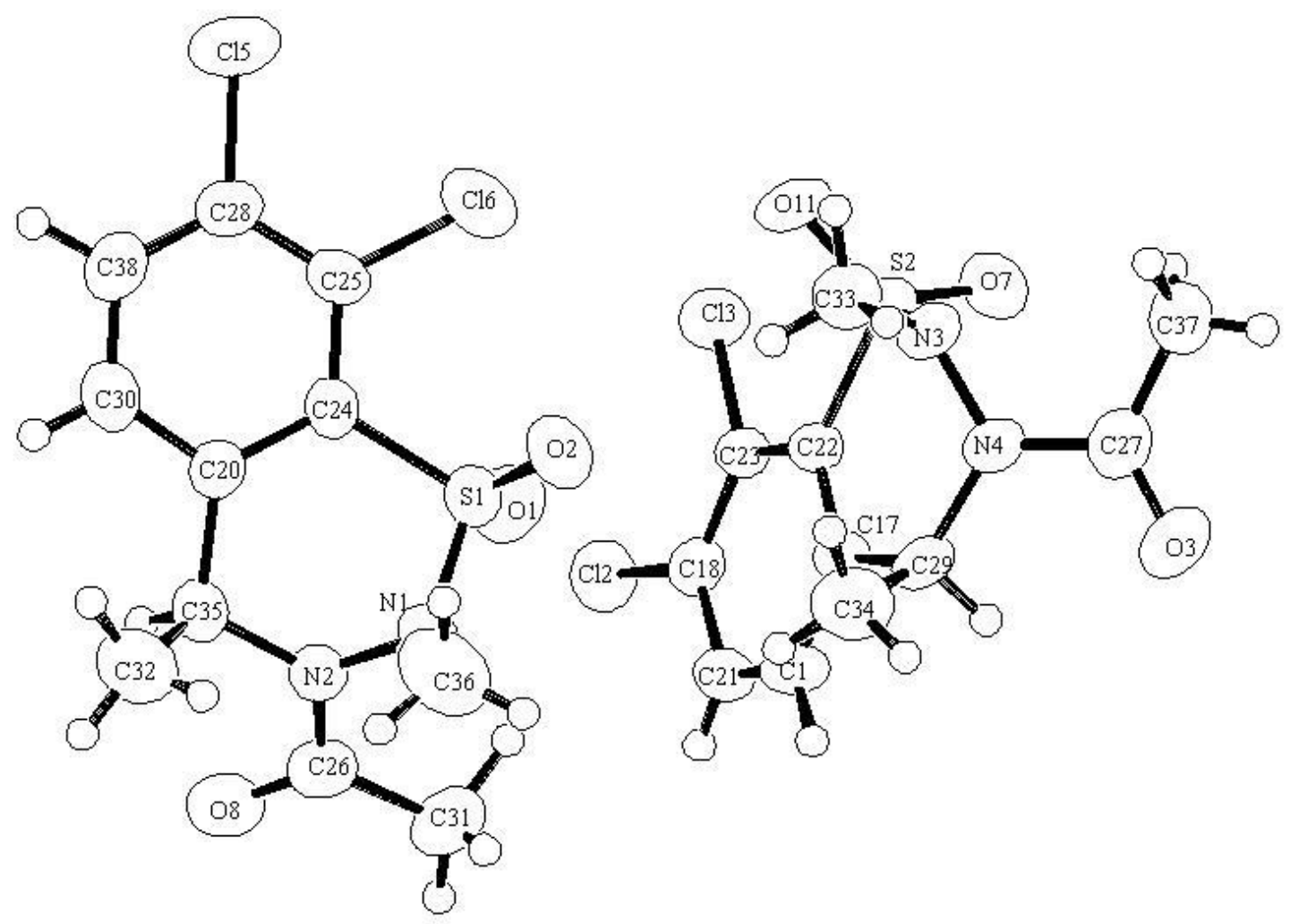

Figure S11. ORTEP plot of 1a with $50 \%$ probability

Table S4. Crystal parameters of 1a

\begin{tabular}{|c|c|}
\hline Space group & P-1 (\#2) \\
\hline$a[\AA]$ & $9.8457(7)$ \\
\hline $\mathrm{b}[\AA]$ & 10.1492(7) \\
\hline$c[\hat{A}]$ & $15.5653(10)$ \\
\hline$\alpha\left[^{\circ}\right]$ & $100.491(4)$ \\
\hline$\beta\left[^{\circ}\right]$ & $100.007(4)$ \\
\hline $\mathrm{y}\left[^{\circ}\right]$ & $109.544(4)$ \\
\hline $\mathrm{V}\left[\mathrm{A}^{3}\right]$ & $1394.27(16)$ \\
\hline density $\left[\mathrm{g} / \mathrm{cm}^{3}\right]$ & 1.540 \\
\hline
\end{tabular}




\section{3a $\cdot \mathrm{H}_{2} \mathrm{O}(\mathrm{CCDC} 1995298)$}

The crystal was grown from methanol by allowing a solution of the compound in a vial to evaporate slowly.

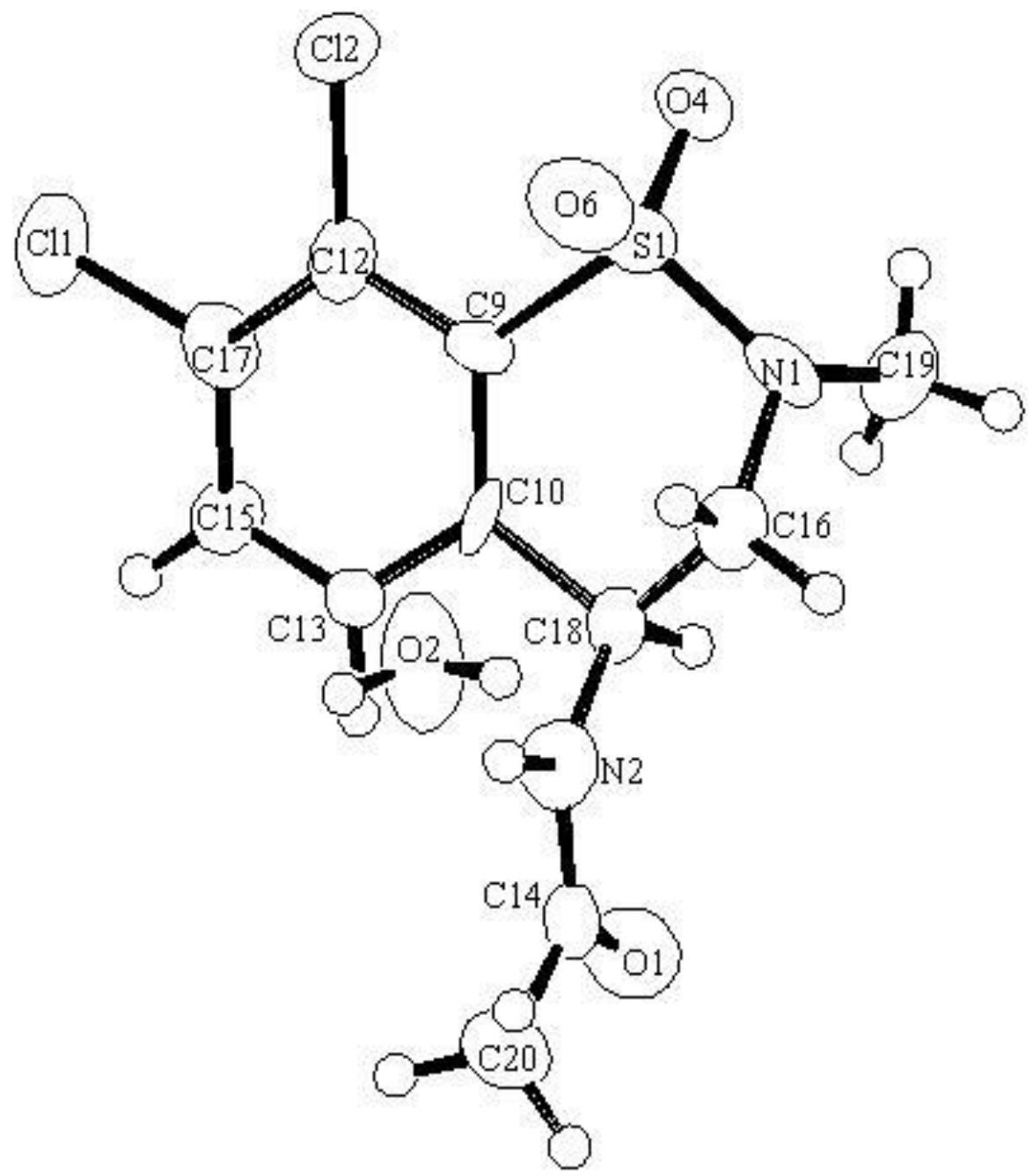

Figure S12. ORTEP plot of $3 \mathbf{a} \cdot \mathrm{H}_{2} \mathrm{O}$ with $50 \%$ probability

Table S5. Crystal parameters of $3 \mathrm{a} \cdot \mathrm{H}_{2} \mathrm{O}$

\begin{tabular}{|c|c|}
\hline Space group & P42bc $(\# 106)$ \\
\hline$a[\tilde{A}]$ & $20.0729(4)$ \\
\hline $\mathrm{c}[\dot{\AA}]$ & $7.2451(2)$ \\
\hline $\mathrm{V}\left[\dot{\AA}^{3}\right]$ & $2919.21(12)$ \\
\hline density $\left[\mathrm{g} / \mathrm{cm}^{3}\right]$ & 1.553 \\
\hline
\end{tabular}

